\title{
Object Localization Using RF Based IEEE 802.11 Frame Transceiving with FER Based RSSI Controlled Calibration
}

\author{
by
}

Gorla Praveen

A thesis submitted in partial fulfillment of the requirements for the degree of Master of Engineering in

Telecommunications

$\begin{array}{ll}\text { Examination Committee: } & \text { Dr. Teerapat Sanguankotchakorn (Chairperson) } \\ & \text { Dr. Poompat Saengudomlert } \\ & \text { Dr. Attaphongse Taparugssanagorn }\end{array}$

Nationality: Indian

Previous Degree: Bachelor of Technology in Electronics and Communications Engineering

Jawaharlal Nehru Technological University, Hyderabad,

Telangana, India

Scholarship Donor: AIT Fellowship

Asian Institute of Technology

School of Engineering and Technology

Thailand

May 2019 


\section{ACKNOWLEDGEMENTS}

I am grateful to many people those who have helped and supported me in gaining knowledge and experience for the completion of my graduation. First, I would like to express my profound gratitude to my esteemed advisor, Dr. Teerapat San guankotchakorn for his advice throughout my master's course and thesis. It is also a great pleasure for me to thank my co-advisor, Dr. Poompat Saengudomlert for providing hardware and timely guidance in the methodological development of the thesis. I also take this space to extend my sincere gratitude to my committee member, Dr. Attaphongse Taparugssanagorn for his comments on improving the thesis.

Secondly, I take this opportunity to convey many thanks to Ms. Premma Rao and Mr. Rajesh Kumar Dehury for their timely act on providing hardware and lab infrastructure in the Telecommunication Engineering department for doing experimentation. In addition, a grateful thanks are extended to the Asian Institute of Technology, Thailand for providing AIT fellowship to support my masters.

Finally, I remember Dr. L. Pratap Reddy, Professor, JNT University Hyderabad, India because of whom my focus towards open research is improved from while I being in my undergraduate. 


\begin{abstract}
This thesis presents an approach of integrating single board computers as an access point and as a short-range object localization system. This is achieved by using open hardware and implementing IEEE $802.11 \mathrm{a} / \mathrm{g} / \mathrm{p}$ based transceiving on low-cost Adlam Pluto Software Defined Radio (SDR), which operates in the S-band frequency range with Frame Error Rate (FER) controlled calibration. Experiments are conducted to investigate the effect of FER on the Received Signal Strength Indicator (RSSI) of IEEE $802.11 \mathrm{a} / \mathrm{g} / \mathrm{p}$ transceiving, there by drawing the connection between the RSSI and the FER. Object localization is done based on the referenced RSSI and the real-time RSSI with FER based controlled calibration of RSSI. About $80 \%$ of accuracy can be expected from the implemented object localization system.
\end{abstract}




\section{TABLE OF CONTENTS}

CHAPTER TITLE

PAGE

Title Page

Acknowledgments

Abstract

Table of Contents

List of Figures

List of Tables

List of Abbreviations

List of Symbols

1 INTRODUCTION

1.1 Overview

1.2 Problem Statement

1.3 Objectives

1

1.4 Limitations and Scope

2

1.5 Organization of the report

LITERATURE REVIEW

2.1 Bistatic Radar and Bistatic Range 3

2.2 Kalman Filter 4

2.3 OFDM Signal Structure 4

2.4 Methods for object localization and detection using Raspberry $\mathrm{Pi}$

2.5 Methods for object localization and detection using RF signals 5

2.6 Additional parameters for human body detection 9

$\begin{array}{ll}2.7 \text { Current state of the research } & 10\end{array}$

$\begin{array}{lll}2.8 \text { Discussion } & 12\end{array}$

3 METHODOLOGY 13

$\begin{array}{ll}3.1 \text { Introduction } & 13\end{array}$

3.2 Power Analysis of Wireless Signals 13

3.3 Object Localization using Wireless Access Point 14

3.4 IEEE $802.11 \mathrm{a} / \mathrm{g} / \mathrm{p}$ based object Localization with RSSI Calibration 17

$\begin{array}{ll}3.5 \text { Hardware } & 22\end{array}$

$\begin{array}{lll}3.6 & \text { Software } & 24\end{array}$

$\begin{array}{ll}3.7 \text { Proposed Model } & 24\end{array}$

$4 \quad$ EXPERIMENTATION RESULTS 26

$\begin{array}{lll}4.1 & \text { Introduction } & 26\end{array}$ 
4.2 Power Analysis $\quad 26$

4.3 Wireless Access Point Localization 30

4.4 RSSI Based on IEEE 802.11 a/g/p implementation 36

5

CONCLUSION

References

Appendices 


\section{LIST OF FIGURES}

FIGURE

2.1

2.4

2.5

2.6

2.7

2.8

2.9

3.1

Block diagram of bistatic range (Willis, Nicholas. 2007. Bistatic Radar )

Ambiguity function diagram (Kelvin Chetty et al., 2009) 6

Range resolution (Chetty et al., 2009)

Doppler resolution (Chetty et al., 2009)

Clutter (static environment) response (Chetty et al., 2009)

Target response (Chetty et al., 2009)

FMCW (Adib et al., 2013)

Basic model using signal generator and spectrum analyzer for feasibility test (Tx and Rx antennas of $2.4 \mathrm{GHz}$ are available in AIT TC Lab)

Algorithmic flowchart based on Wireless Access Point

Implementation of realtime RSSI receiving and calibration for IEEE $802.11 \mathrm{a} / \mathrm{g} / \mathrm{p}$ localization

Localization Estimation

Basic Model of Raspberry pi (Raspberry Pi foundation., 2016)

ADALM-PLUTO (Analog Devices., 2017)

Proposed Hardware model

Connection diagram for proposed model

Power Analysis Experimentation Scenario

Power Analysis Experimentation 1

Power Analysis Experimentation 2

Actual distance vs. estimated distance for every one-meter range from experiment 1

Actual distance vs. estimated distance for every one-meter range from experiment 2

Actual distance vs. estimated distance for every one-meter range to the left and right of the AP from experiment 1

Actual distance vs. estimated distance for every one-meter range to the left and right of the AP from experiment 2 
4.14 Kalman filtered velocity and real-time velocity 35

4.15 Frame Error Rate Analysis 36

4.16 FER and Distance 38

$\begin{array}{lll}4.17 & \text { FER vs. RSSI Variance } & 39\end{array}$

4.18 FER vs. RSSI 40

4.19 Actual distance vs. estimated distance with and without FER based correction

4.20 Actual distance and estimated error with and without FER based correction

4.21 Actual distance and estimated error percentage with and without $F E R$ based correction

4.22 Error percentage in distance estimation with and without FER 43

$4.23 \quad$ Experimentation setup at various stages 44

4.24 Tx moving away from Rx1 and Rx2 45

4.25 Distance estimated by Rx1 for Tx as shown in figure 4.24 46

4.26 Distance estimated by Rx2 for Tx as shown in figure 4.24 46

4.27 Tx is moved from Left to Right 47

4.28 Distance estimated by Rx1 when Tx is moved as shown in figure 4.27 47

$4.29 \quad$ Obstacle between Tx and Rx 48

4.30 Distance estimated by Rx1 for Tx moving as shown in figure $4.29 \quad 48$

4.31 Distance estimated by Rx2 for Tx moving as shown in figure $4.29 \quad 49$

A.1 Working Images $\quad 93$

$\begin{array}{lll}\text { A.2 Working Images } & 94\end{array}$ 


\section{LIST OF TABLES}

TABLE TITLE

PAGE

$2.1 \quad$ Kalman Table 4

2.2 ACS values of human body deduced from the literature 10

3.1 Key parameters 14

4.1 Probability of FER Occurrence $\quad 37$

$\begin{array}{lll}4.2 & \text { Probability of FER Occurrence } & 37\end{array}$

$4.3 \quad \Delta$ RSSI Calibration Values 40

4.4 Error percentage in distance estimation with and without FER 43 


\section{LIST OF ABBREVIATIONS}

\begin{tabular}{|c|c|}
\hline $\mathbf{A P}$ & Access Point \\
\hline ACR & Absorption Cross Section \\
\hline CFR & Channel Frequency Response \\
\hline CIR & Channel Impulse Response \\
\hline CS & Compressive Sensing \\
\hline CSI & Channel State Estimation \\
\hline DBPSK & Differential Binary Phase Shift Keying \\
\hline DHCP & Dynamic Host Configuration Protocol \\
\hline DNS & Domain Name System \\
\hline DQPSK & Differential Quadrature Phase Shift Keying \\
\hline DSI & Direct Signal Interference \\
\hline DSSS & Direct Sequence Spread Spectrum \\
\hline FER & Frame Error Rate \\
\hline FMCW & Frequency-Modulated Continuous-Wave \\
\hline FPGA & Field-Programmable Gate Array \\
\hline GNU & GNU's NOT Unix \\
\hline GPS & Global Positioning System \\
\hline IEEE & Institute of Electrical and Electronics Engineers \\
\hline LOS & Line of Sight \\
\hline MAC & Media Access Controller \\
\hline MHz & Megahertz \\
\hline NLOS & Non Line of Sight \\
\hline NLR & Nonlinear Regression \\
\hline OFDM & Orthogonal Frequency Division Multiplexing \\
\hline PHY & Physical Layer \\
\hline PLCP & Physical Layer Convergence Protocol \\
\hline PPDU & PLCP Protocol Data Unit \\
\hline QAM & Quadrature Amplitude Modulation \\
\hline RADAR & RAdio Direction And Ranging \\
\hline $\mathbf{R F}$ & Radio Frequecny \\
\hline RSSI & Received Signal Strength Indicator \\
\hline SDR & Software Defined Radio \\
\hline SAR & Specific Absorption Rate \\
\hline
\end{tabular}


SSH Secure Shell

SSID Service Set Identifier

TOF Time of Flight

WAP Wireless Access Point

WC-CWL Weighted Centroid - Constrained Weighted Least Square

Wi-Fi Wireless Fidelity

WPA Wi-Fi Protected Access 


\section{LIST OF SYMBOLS}

$\begin{array}{ll}c & \text { Velocity of light } \\ D_{r x} & \text { Distance between } R_{x} \text { and target } \\ D_{t x} & \text { Distance between } T_{x} \text { and target } \\ f_{d} & \text { Frequency shift } \\ n & \text { Path loss component } \\ p(x) & \text { Probability of } x \\ R x & \text { Receiving antenna } \\ T x & \text { Transmitting antenna } \\ \delta f & \text { Change in frequency } \\ V & \text { Velocity of target } \\ \chi & \text { Basic ambiguity function } \\ \tau & \text { Time delay } \\ \Delta R S S I & \text { Change in } R S S I\end{array}$




\section{CHAPTER 1}

\section{INTRODUCTION}

\subsection{Overview}

Indoor localization and detection of objects using wireless signals (Adib et al., 2014) has been complex issue because of the closed architecture which does not allow customizable system design. Two models are considered in this thesis, one at the primary level of research and other at the secondary level of research. At the primary level, the focus is on detection of objects with efficient algorithms using wireless signals and digital signal processing. At the secondary level, the focus is on implementing the above said concept on an Open Router as an Access Point for object localization with the existing Open Hardware and with the implementation of IEEE $802.11 \mathrm{a} / \mathrm{g} / \mathrm{p}$ based transceiving on low cost Adlam Pluto Software Defined Radio (SDR), which operates in the S-band frequency range, with Frame Error Rate (FER) controlled calibration. There are several models demonstrated by research scholars for target detection and indoor localization using wireless signals (Guo et al., 2008). This thesis demonstrates the use of single board computers which has routing capabilities as an Access Point and SDR for target detection using wireless signals.

\subsection{Problem Statement}

Indoor localization in this era of Internet of Things has been on the rise to detect objects with high accuracy. But it needs a large number of sensors and at least one networking device (router) to be employed for object sensing and data communications. Existing methods include the use of RSSI and Channel State Information (CSI) from the reflected signals where external WiFi access points (AP) act as a transmitters along with reference antennas and surveillance antennas for reception (Chetty et al., 2009). Other methods are based on calculating time of flight (ToF) using a chirp generator with transmitting and receiving antennas (Adib et al., 2014). However the use of RSSI still lacks calibration when there are occurrences of frame errors for IEEE $802.11 \mathrm{a} / \mathrm{g} / \mathrm{p}$ devices. This proposal is a novel approach of integrating object detection in router acting as an Access Point with controlled calibration of IEEE $802.11 \mathrm{a} / \mathrm{g} / \mathrm{p}$ RSSI based object localization. The implementation employs single board computers and software defined radio (SDR). 


\subsection{Objectives}

- To design a system for indoor localization using wireless signals.

- To implement indoor localization in a router (Wireless Access Point) using single board computer(s).

- To implement indoor localization based on Adlam Pluto SDR for IEEE $802.11 \mathrm{a} / \mathrm{g} / \mathrm{p}$ transceiving with FER controlled calibration.

\subsection{Limitations and Scope}

The developed system is used to detect and localize objects such as IEEE $802.11 \mathrm{a} / \mathrm{g} / \mathrm{p}$ devices with FER based RSSI calibration at short-range distances. Due to low processing capabilities of low-cost Software Defined Radios and single board computers, the accuracy is moderate. The developed system can be extended to detect and localize different objects for automation in the Internet of Things (IoT) domain.

\subsection{Organization of the report}

Chapter 1: Introduction: Overview, problem statement, objective, scope and limitations and organization of the report.

Chapter 2: Literature review on the current state of research.

Chapter 3: Research methodology and the expected outcome.

Chapter 4: Experimentation results and analysis.

Chapter 5: Conclusion on current and future scope of the research. 


\section{CHAPTER 2}

\section{LITERATURE REVIEW}

There has been numerous development in radar systems for localization from the past which has led us to improve more refined systems for indoor localization. Earlier radar systems are developed especially for military purpose but now research has expanded to develop tiny system at affordable cost with low range object detection for localization. The following represents some of the methods and the current state of the research.

\subsection{Bistatic Radar and Bistatic Range}

Bistatic radar as shown in Figure 2.1, is a type of radar system in which the distance between the transmitter and the receiver is almost correspondingly comparable to the approximated target range. In opposite to this there are other radar systems such as Pseudo-monostatic radar, Forward scatter radar, Multistatic radar and passive radar where these are different from Bistatic radar in a way of separate Tx \& Rx, range of detection, principle of detection, type and number of antennas used.

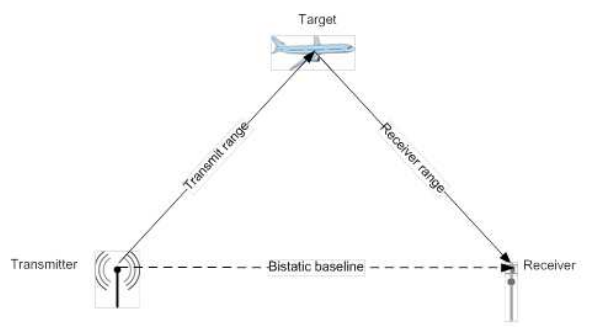

Figure 2.1: Block diagram of bistatic radar (Nick Young. NATS, 2017 )

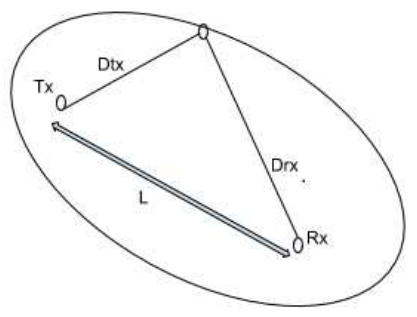

Figure 2.2: Block diagram of bistatic range (Willis, Nicholas. 2007. Bistatic Radar) 
Basic determination of range using separate transmitter and receiver by calculating time of arrival of the object is called as bistatic range. Bistatic range as shown in Figure 2.2 is equivalent to $D_{t x}+D_{r x}-L$ where $D_{t x}, D_{r x}$ and $L$ are distances between transmitter and target object, target object and receiver, and transmitter and receiver respectively (Willis, Nicholas. 2007) .

\subsection{Kalman Filter}

Table 2.1: Kalman Table

\begin{tabular}{|l|l|l|}
\hline State Prediction & $X_{\text {predicted }}=A X_{n-1}+B U_{n}$ & Next output prediction \\
\hline Covariance Prediction & $P_{\text {predicted }}=A P_{n-1} A^{T}+Q$ & Next error prediction \\
\hline Innovation & $\bar{Y}=Z_{n}-H X_{\text {predicted }}$ & $\begin{array}{l}\text { Difference between reality } \\
\text { and prediction }\end{array}$ \\
\hline Kalman Gain & $S=H P_{\text {predicted }} H^{T}+R$ & $\begin{array}{l}\text { Difference between real } \\
\text { error and predicted error }\end{array}$ \\
\hline State Update & $K=P_{\text {predected }} H^{T} S^{-1}$ & Kalman Gain calculation \\
\hline Covariance Update & $X_{n}=X_{\text {predicted }}+K \bar{Y}$ & Update estimate \\
\hline
\end{tabular}

Kalman filter, as a linear quadratic equation that tracks the estimated state and variance of the system. There by considering state transition model an updated and more accurate value of estimate is resulted. Parameters such as $X_{n-1}$ (previous state estimate) and $P_{n-1}$ (previous state error) are taken in to consideration along with $\bar{Y}$ (Innovation) and K(Kalman Gain) to often result an accurate estimate.

Extended models such as extend Kalman filter and unscented Kalman filter to overcome the failure of Kalman filter in accurate estimation of a state in non-linear systems.

\subsection{OFDM Signal Structure}

Software Defined Radio based IEEE $802.11 \mathrm{a} / \mathrm{g} / \mathrm{p}$ uses OFDM signal structure for frame transceiving. The following frame represented in figure 2.3 contains data which is OFDM encoded with variable length 
with varying payload between 0 - 4095 bytes. OFDM uses guard intervals (GI) in between long preamble, Signal field, Service and payload.

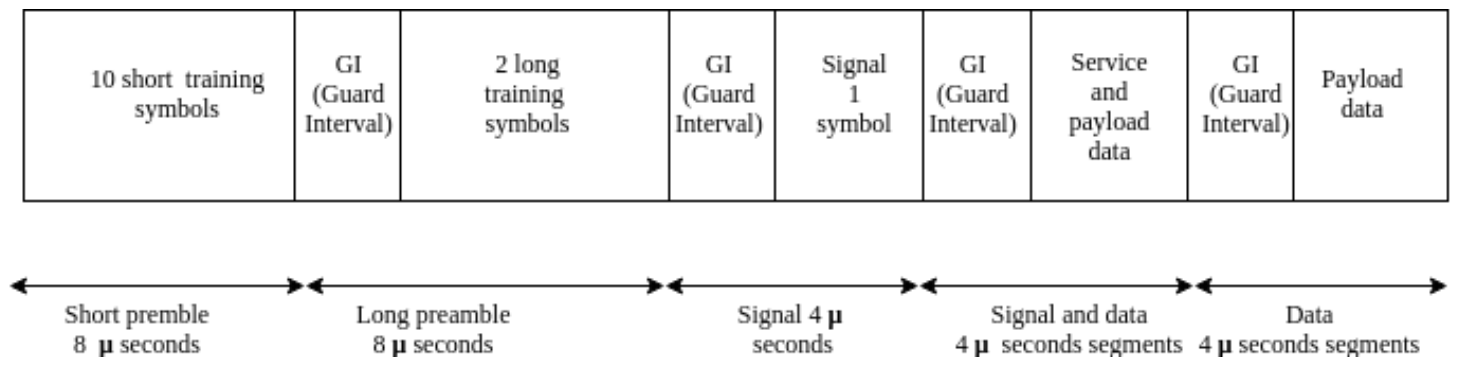

Figure 2.3: OFDM Signal Structure

\subsection{Methods for object localization and detection using Raspberry Pi}

\section{Raspberry Pi and digital camera}

Raspberry $\mathrm{Pi}$, a single board computer is a great tool to interface with digital cameras for image processing techniques. Raspberry $\mathrm{Pi}$ as a low cost medium performance microprocessor is capable of processing vision applications and is extensively used for surveillance as it finds easier interface with cameras. As described by Abaya et al., 2014 it is possible to realize low cost smart security camera with night vision using Raspberry and Open CV along with infrared(IR) led.

Basic system includes use of IR for activating night mode vision of webcam when there is a stance of an object presence. Raspberry pi acts as a image processing unit and upon processing along with noise elimination by background subtraction. Here background subtraction helps in detecting moving objects by frame difference method. it imports data to web server where a person away from home can access this data anywhere. There are many applications of Raspberry Pi in detecting object using digital cameras such as in Islam et al., 2014, Raspberry $\mathrm{Pi}$ is used as an image processing tool in electronic voting machine for detecting face. It is also used in Pereira et al., 2014 for object sorting by a robotic arm and placing the object in desired position and moreover in Janard et al., 2015 it is used for real time detection of miscellaneous object and to follow them by using telepresence robots.

\subsection{Methods for object localization and detection using RF signals}

\section{Ambiguity function}

Ambiguity function analysis is mainly used in radar engineering to establish relation between transmitted signal and the received signal which describes extent of distortion between transmitted and received signal. But many definitions of ambiguity function exist such as describing propagation delay and Doppler shift in wideband signals. However basic definition is to describe the relation between two signals in time and frequency domain. 
It is a two- dimensional functional analysis of time delay and Doppler shift in frequency of moving object. Where Equation 2.1 represents self ambiguity function of a signal, i.e the relation between a signal $\left(x_{1}(t)\right)$ and its time delayed $(\tau)$ version with frequency shift $\left(f_{d}\right)$. Equation 2.2 represents cross ambiguity function between transmitted signal $\left(x_{1}(t)\right)$ and received reflected signal $\left(x_{2}(t)\right)$ from the moving target object with time delay $(\tau)$ and Doppler frequency shift $\left(f_{d}\right)$.

$$
\begin{aligned}
& \left|\chi_{s}\left(\tau, f_{d}\right)\right|=\left|\int_{-\infty}^{\infty} x_{1}(t) x_{1}^{*}(t-\tau) \exp \left(j 2 \pi f_{d} t\right)\right| \\
& \left|\chi_{c}\left(\tau, f_{d}\right)\right|=\left|\int_{-\infty}^{\infty} x_{1}(t) x_{2}^{*}(t-\tau) \exp \left(j 2 \pi f_{d} t\right)\right|
\end{aligned}
$$

Further this ambiguity function can be described for zero Doppler shift $\left(f_{d}=0\right)$, i.e when the target object is stationary, as a correlation between $\left(x_{1}(t)\right)$ and $\left(x_{2}(t)\right)$ as $\chi_{c}(\tau, 0)$.

Ambiguity function graph in figure 2.4 from Kelvin Chetty et al., 2009 represents normalized amplitude $\left|\chi_{c}\left(\tau, f_{d}\right)\right|$ in $z$-axis, Doppler shift $\left(f_{d}\right)$ in y-axis and delay $(\tau)$ expressed in terms of distance $(m)$ from which target object is located from the system. So normalized amplitude $\left|\chi_{c}\left(\tau, f_{d}\right)\right|$ shows extent of distortion between transmitted signal and the received signal.

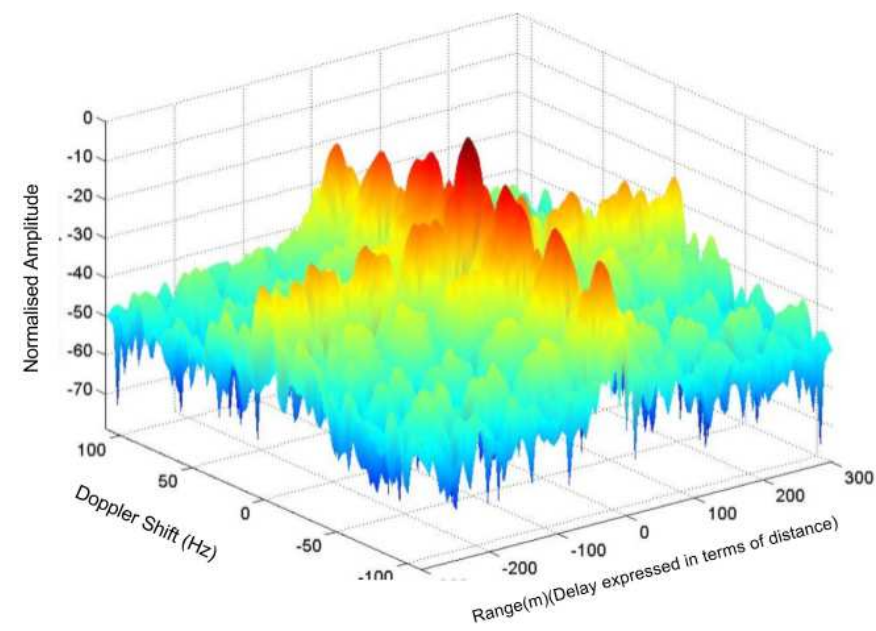

Figure 2.4: Ambiguity function diagram (Kelvin Chetty et al., 2009)

\section{Data reception and data processing}

1. When a signal is transmitted with certain frequency and the reception of signal is done through receiving antenna which configured to receive for some time. All the data is processed in MATLAB using cross ambiguity function. 
2. For determining range resolution, taking for zero Doppler cut i.e $f_{d}=0$. Graph between normalized amplitude and range is done as an example shown in figure 2.5 from Kelvin Chetty et al., 2009 where range resolution is determined.

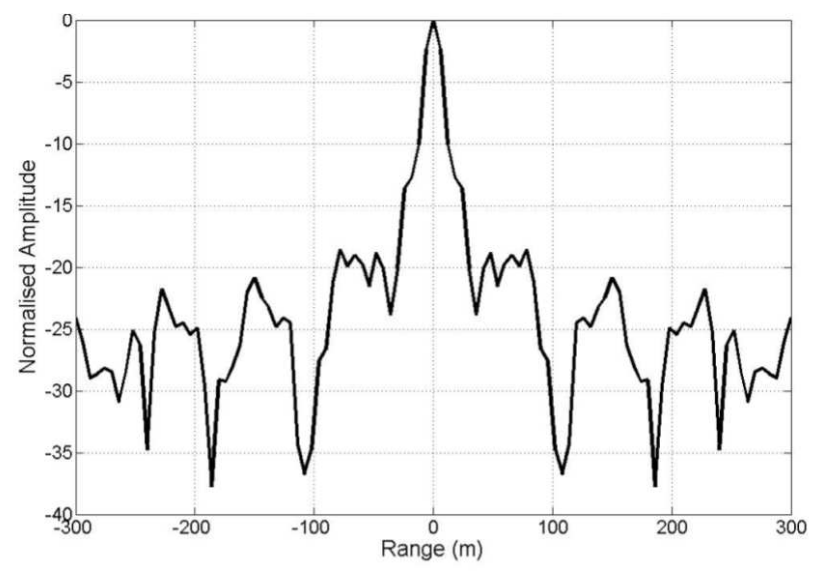

Figure 2.5: Range resolution (Chetty et al., 2009)

3. For determining Doppler resolution, considering zero range cuts. Graph between normalized amplitude and Doppler shift is obtained as an example shown in figure 2.6 from Chetty et al., 2009 where Doppler shift resolution is determined.

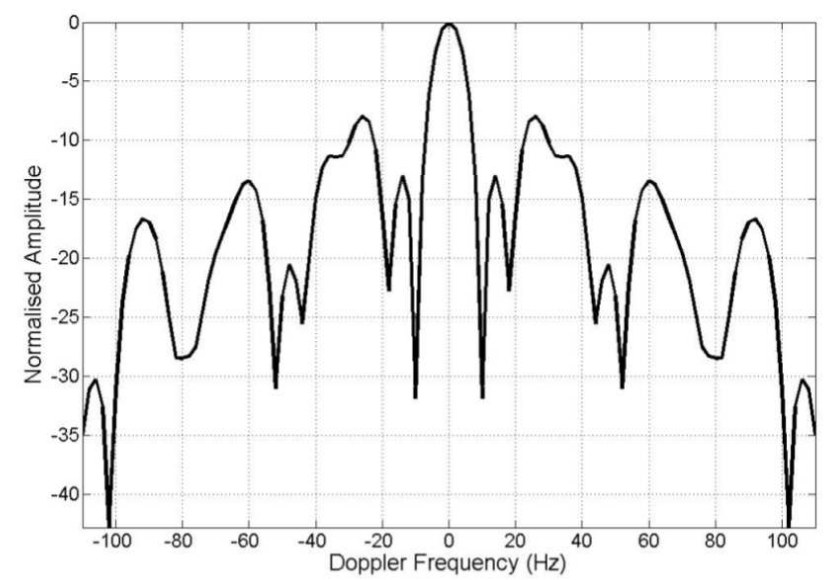

Figure 2.6: Doppler resolution (Chetty et al., 2009)

4. Then Doppler-range graphs are obtained when the reception is done and when there is no presence of object. Here the received signal is prone to Direct Signal interference (DSI) and static surrounding environment.

5. Through DSI cancellation, i.e subtracting self ambiguity from the cross ambiguity and again plotting Doppler-range graph as a example shown in figure 2.7 which is an elimination of DSI but with the presence of static environment (Clutter). 


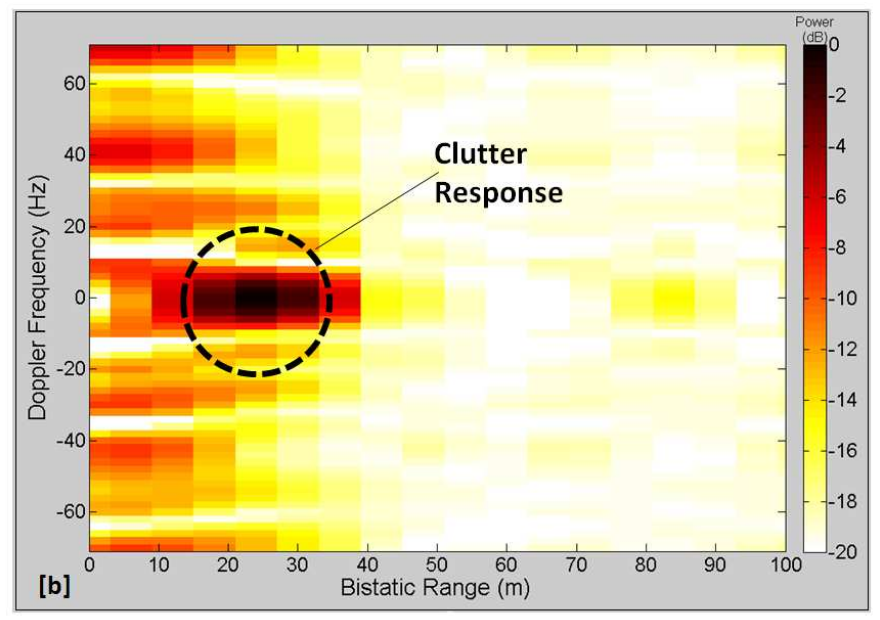

Figure 2.7: Clutter (static environment) response (Chetty et al., 2009)

6. When the target is made to present and data reception is done for some time along with DSI cancellation. Doppler - range graph are obtained. By analyzing the Doppler-range graphs from (5) and (6) with the shift in Doppler and range makes us to distinguish absence and presence of object

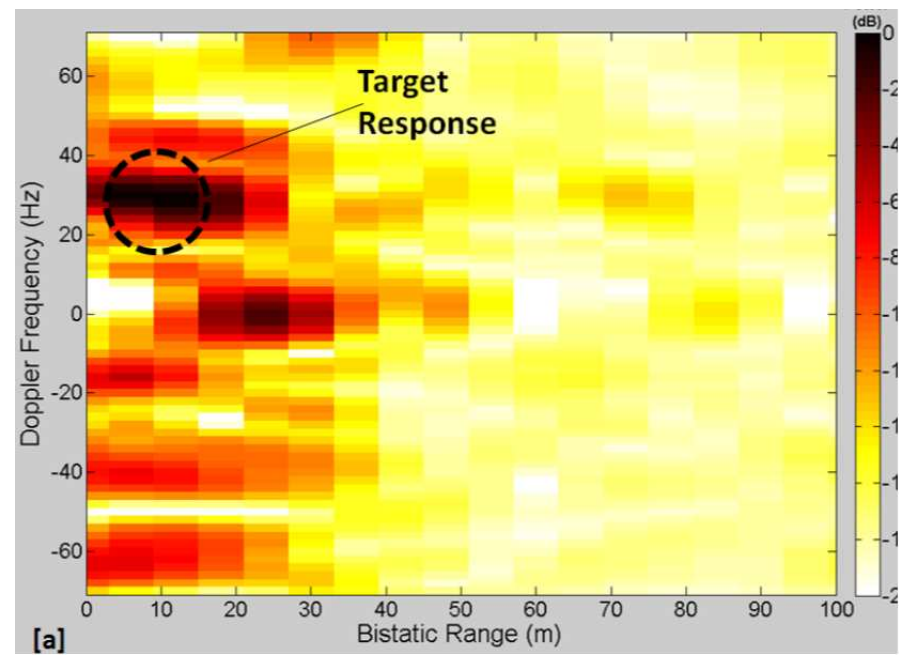

Figure 2.8: Target response (Chetty et al., 2009)

\section{From FMCW generator}

Frequency Modulated Carrier Wave which is a periodic RF signal with linear increase of frequency in a time as shown in figure 2.9. The transmitted signal reflects after some time form the target which is present in the room, in which reflected is received by FMCW receiver. Here the time of flight is used for determination of 1 of object from the FMCW system. 


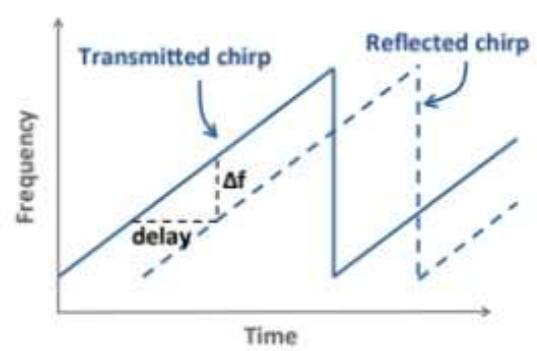

Figure 2.9: FMCW (Adib et al., 2013)

Range resolution which is a minimum measurable change in location with minimum measurable shift in frequency $\delta f_{\text {min }}$ is represented in Equation 2.3 where delay is the Time of Flight (TOF) with minimum TOF as $T O F_{\min }$ of a signal which is reflected back to the receiver upon transmitted from transmitter when there is a presence of target, $\delta f$ is the instantaneous differences in frequency at transmitter and receiver, $\mathrm{C}$ is the speed of light and factor 2 accounts for the twice of distance between system and target where the signal travelled from transmitter to target and from target to receiver.

$$
\text { Resolution }=C \times \frac{T O F_{\min }}{2}=C \times \frac{\delta f_{\min }}{2(\text { slope })}
$$

1. In order to distinguish between presence and absence of object. Initially, we made to work transmission of FMCW and its reception of reflected wave when there is no presence of object and with static environment.Thus considering this as a background noise. The data such as TOF corresponding to each frequency is noted.

2. Now the target such as human body is made to present and the data such as TOF is recorded. Then after subtracting background noise, we could able to distinguish the presence of absence with a distance of $D$ as represented in the Equation 2.4 from the system.

$$
D=C \times T O F
$$

(Equation 2.4)

\subsection{Additional parameters for human body detection}

Human body responses at different levels at different frequencies. In order to study the reflection parameters and to deduce the parameters for detection we need certain parameters like absorption rate. One such parameter is Specific Absorption Rate $S A R$, which defines the amount of absorbed power(in Watts) of an EM signal per unit mass (of human body). Relation is shown in Equation 2.5

$$
S A R=\frac{P_{\text {owerabsorbed }}(W)}{\text { mass }}
$$

The other most accurate form is to define Absorption Cross Section $A C S$, as amount of power absorbed 
by the body per unit power density $S_{c}$ of an incident signal. Relation is as shown in Equation 2.6

$$
A C R\left(\sigma_{a}\right)=\frac{P_{\text {owerabsorbed }}(W)}{S_{c}\left(W / m^{2}\right)}
$$

The following parameters as shown in the Table 2.2 represents ACS values of the human body at different frequencies at differnet scenarios (Melia, 2013.)

Table 2.2: ACS values of human body deduced from the literature

\begin{tabular}{|c|c|c|c|c|}
\hline Authors & $f(\mathrm{GHz})$ & Method & Subject & $\operatorname{ACS}\left(m^{2}\right)$ \\
\hline Andersen et al. & $3-8$ & Exp & Several & 0.33 \\
\hline Bamba et al. & $2.4-3.0$ & Exp. & $\begin{array}{l}\text { Several avg } 70 \mathrm{~kg} \\
175 \mathrm{~cm}\end{array}$ & $0.34,0.36$ \\
\hline Harima et al. & $1-4$ & Exp & $70.6 \mathrm{~kg}$ male & $0.33-0.11$ \\
\hline Hirata et al & 2 & FDTD sim. & Japanese adult child & $04-0.5$ \\
\hline Hurst et al. & 2.1 & Not stated & Unknown & 0.4 \\
\hline Robinson et al. & 0.91 & TLM sim. & thin boundary models & 0.25 \\
\hline Uusitupa et al & $0.9-5$ & $\operatorname{sim}$ & VF Male $(72.2 \mathrm{~kg})$ & $0.4-0.25$ \\
\hline Findaly et al. & $1-2$ & FDTD sim. & NORMAN (73.0 kg) & $0.35-0.3$ \\
\hline Kientega et al. & 2.4 & FDTD sim. & $\begin{array}{l}\text { Thelonius (1.17m, } 19 \\
\mathrm{~kg})\end{array}$ & $0.06-0.14$ \\
\hline
\end{tabular}

\subsection{Current state of the research}

- Guo et al., 2008 - This is the first experiment to detect objects with Wi-Fi signals by using Beacon transmissions such as two parts of DBPSK and DQPSK with DSSS modulation used for transmission for wireless based passive radar. Self ambiguity function analysis and cross ambiguity analysis is done upon reception of signals for detection.

- Ahamed et al., 2008 - Tracking system for pervasive environment, linear approximation with RSSI is used by collecting samples by measuring distance and direction of Wi-Fi APs.

- Chetty et al., 2009 - Detection of target using Passive Bistatic Wi-Fi radar when system and target are in high densified echo environment. By using Wi-Fi Access point, reference receiver and 
surveillance receiver with OFDM, 64 QAM transmission, ambiguity analysis is done for location approximation using bistatic radar concept with nullification of DSI (Direct Signal Interference). A similar work by the same author (Kevin Chetty et al., 2011) is through the wall sensing of personnel using passive bistatic Wi-Fi radar at standoff distances. In this targets are monitored from outside the wall so as to detect the target satisfying the concept of seeing through the walls.

- Falcone et al., 2012 - Tracking of non stationary objects is done with Wi-Fi based radar by using Global Positioning System (GPS) for accurate positioning.

- Rzewuski and kulpa, 2011 -Designing of system concept for localization using PPDU (PLCP Protocol data Unit) of MAC address to locate the destination.

- Zhou et al., 2015 - Explained the concept of using Channel State Information (CSI), a PHY feature for extracting Channel Frequency Response (CFR) and Channel Impulse Response (CIR) with amplitude and phase detection of the reflected signal. CSI can be obtained from the Wi-Fi network cards with a constraint of using CSI of only $40 \mathrm{MHz}(802.11 \mathrm{n})$ bandwidth.

- Wu et al., 2015 - Examined the use of CSI for tracking humans, phase and frequency diversity is used to map body with use of chest motion of human. Experiment is done for LOS and NLOS detection.

- Van Dijk et al., 2008 - Design of FMCW antenna Array for radar applications with independency in transmitter and receiver reception with Direct Digital Synthesis Technology.

- Adib et al., 2013 - See-Through walls with Wi-Fi, a wall gesture based communication using inverse sympathetic detection (using only one receiver). Algorithm based detection by calculating the correlation matrix and eigenvalues for separating the signal from the noise along with indication of direction.

- Adib et al., 2014 - Based on RF Reflections of the body by using static frequency modulated continuous wave (FMCW) where different parts of body respond for reflecting signals at different frequencies. Here FMCW is operated for some duration, so different body parts reflect at different frequencies and the shape forming of the body is done.

- Adib et al., 2014 - A similar work by using RF reflections for multi personnel localisation by using multi-shift FMCW.

- Adib et al., 2015 - Based on variations in reflected signal from the stationary human body monitoring of heart beat is done. Without loss in generality that these variations are due to heartbeat reflections of stationary human body at particular reflected frequency of heart with narrow bandwidth.

- Paolo Barsocchi et al., 2007 - This paper demonstartes the commonly used frame loss model for simulation over Wi-Fi channels. It estimates the variance of RSSI with FER.

- Zan Li eta al., 2015 - about study of Passive Wi-Fi source Localization implemented on GNU Radio testbed 


\subsection{Discussion}

Literature review on several existing methods has shown significant development in designing indoor positioning systems. Some of the methods discussed in section 2.7 use RSSI and CSI (Zhou et al., 2015) of the wireless signals. Wireless beacon transmission, such as DSSS \& DBPSK, DSSS \& DQPSK modulated signal described in (Geo et al., 2008), is the first method used for target detection. The transmitted beacon signal changes its characteristics upon reflection from the target, and these changed characteristics are used for signal analysis. In particular, with the use of the ambiguity function, changes in characteristics of the transmitted signal are analyzed and the results can lead to determine the characteristics of a target.

Latest research reveals that the use of a chirp generator described in (Adib et al., 2014, Adib et al., 2015) finds its applications not only in target detection but also in shape forming. When a chrip generator generates signals with varying frequencies from which different human body parts reflect, these reflections are captured by receiving antennas, to be followed by sample collection and signal processing techniques. This method also improves detection accuracy with moderate accuracy in shape forming.

All these systems are capable of detecting a target, but these methods are difficult to integrate with existing systems because of closed system properties and interportability. However, these methods are the foundation for research in radar engineering, especially in indoor localization.

As the RSSI is prone to frame error (Paolo Barsocchi et al., 2007), existing system lack in calibrating the RSSI based on the Frame Error Rate (FER). They also need more infrastructure. Some of the methods use external Wi-Fi APs as transmitters, which increases the system dependency on others and limits the detection accuracy because of unknown exact natures of APs. This thesis contributes to designing a system with the use of Single Board Computers which simultaneously act as a Wi-Fi AP and an object detecting system with the use of SDR calibrating the RSSI based on FER. This system is also relatively low-cost and small in size, with the use of minimal infrastructure and increasing independency. 


\section{CHAPTER 3}

\section{METHODOLOGY}

\subsection{Introduction}

Experimentation methodology involves a basic Power Analysis of Transmitting \& Receiving Signals using Signal Generator \& Oscilloscope as discussed in section 3.2. Then, Access Point based localization is discussed in section 3.3 and finally, object localization using IEEE 802.11 a/g/p Frame Transceiving is implemented on Adalam Pluto SDR with controlled calibration of RSSI based on Frame Error Rate (FER) is discussed in section 3.4

\subsection{Power Analysis of Wireless Signals}

We examine the feasibility of the proposed model by transmitting, and receiving the reflected signal by using available transmitting(Tx) and receiving(Rx) antennas which works in the $\mathrm{MHz}$ up to $\mathrm{GHz}$ frequency ranges. For this we need to do the following to examine the feasibility of establishing consistency in object detection based on received signal levels.

1. Tx Antenna : A transmitting antenna which is readily available for experimentation and which generates from $\mathrm{MHz}$ to $\mathrm{GHz}$ frequencies is needed for physical experimentation.

2. Rx Antenna : An antenna capable of detecting the signal having frequencies from $\mathrm{MHz}$ to $\mathrm{GHz}$ frequencies.

3. Signal generator which is connected to Tx antenna and Oscilloscope connected to Rx antenna as shown in figure 3.1 is used to primarily transmit and receive the signal and later on they are replaced with microprocessor to generate and detect frequencies and process the information. Key parameters that are to be observed are shown in Table 3.1

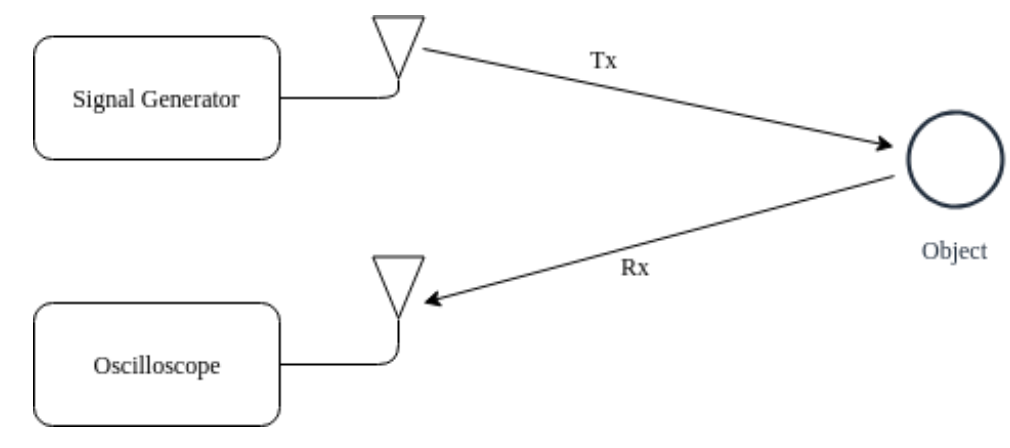

Figure 3.1: Basic model using signal generator and spectrum analyzer for feasibility test (Tx and $\mathrm{Rx}$ antennas of $2.4 \mathrm{GHz}$ are available in AIT TC Lab) 
Table 3.1: Key parameters

\begin{tabular}{|c|}
\hline Key parameters \\
\hline Transmitted power $\left(P_{t x}\right)$ \\
Received power $\left(P_{r x}\right)$ \\
\hline
\end{tabular}

Further process of experimentation and its results are discussed in section 4.2 of chapter 4 are to test feasibility of consistency in object localization based on RSSI.

\subsection{Object Localization using Wireless Access Point}

The following steps involves in making the experiment:

- Access Point Setup : Raspberry Pi is booted with Linux Operating System called Raspbian OS. The following setup is required for Access Point creation.

- Hostapd: It is a WAP software daemon which is used to Setup the Wi-Fi. .

- Installing and configuring the Hostapd and assigning the channel, internal IP range, DHCP, session time, SSID and WPA2 authentication support for Access Point. It also involves configuring DNS and the routing tables for making the system to access the internet through the ethernet.

- Client association with the Access Point

\section{- Client RSSI received reading in Access Point:}

- Client mobile is connected to the Access Point.

- Access Point is accessed through Secure Shell (SSH).

- RSSI receiving: Networking commands are used for finding the RSSI of the particular client.

- Bash scripts are written to automate the process of writing the RSSI values to a script files as shown in figure 4.7 .

\section{- Filtering:}

- Instantaneous value of RSSI is fetched as said above to calculate the localization distance.

- Instantaneous time difference $(\Delta t)$ between two consecutive values is determined.

Consider $x=$ distance in meter $s, \dot{x}=$ instantaneous velocity in meter $/$ second.

$$
\begin{gathered}
\bar{x}=\dot{x} \Delta t+x \\
\bar{x}=\dot{x}
\end{gathered}
$$




$$
\left[\begin{array}{l}
\bar{x} \\
\bar{x}
\end{array}\right]=\left[\begin{array}{cc}
1 & \Delta t \\
0 & 1
\end{array}\right] \times\left[\begin{array}{l}
x \\
\dot{x}
\end{array}\right]
$$

where $\bar{x}$ and $\overline{\dot{x}}$ are predicted values of object position and instantaneous velocity respectively. Equation 3.3 can be re-written as follows as shown in equation Equation 3.4

$$
\left[\begin{array}{c}
P_{\text {predicted }} \\
V_{\text {velocity }}
\end{array}\right]=\left[\begin{array}{cc}
1 & \Delta t \\
0 & 1
\end{array}\right] \times\left[\begin{array}{c}
P_{\text {initial }} \\
V_{\text {initial }}
\end{array}\right]
$$

In Equation $3.4, P_{\text {initial }}$ and $V_{\text {initial }}$ are the previous position and velocity of the object respectively where $P_{\text {predicted }}$ and $V_{\text {predicted }}$ next values after time stamp $\Delta t$. Default distance value of one meter and average velocity of 1.4 meters/second are used as kalman filter default value as describe in section 2.2 with 0.01 as default system noise. This parameters are used in Kalman filter for evaluating the results as shown in figure 4.13 and 4.14 


\subsubsection{Algorithmic Flowchart based on WAP}

The following flow chart as shown in figure 3.2 describes RSSI calibration and distance estimation with Wireless Access Point.

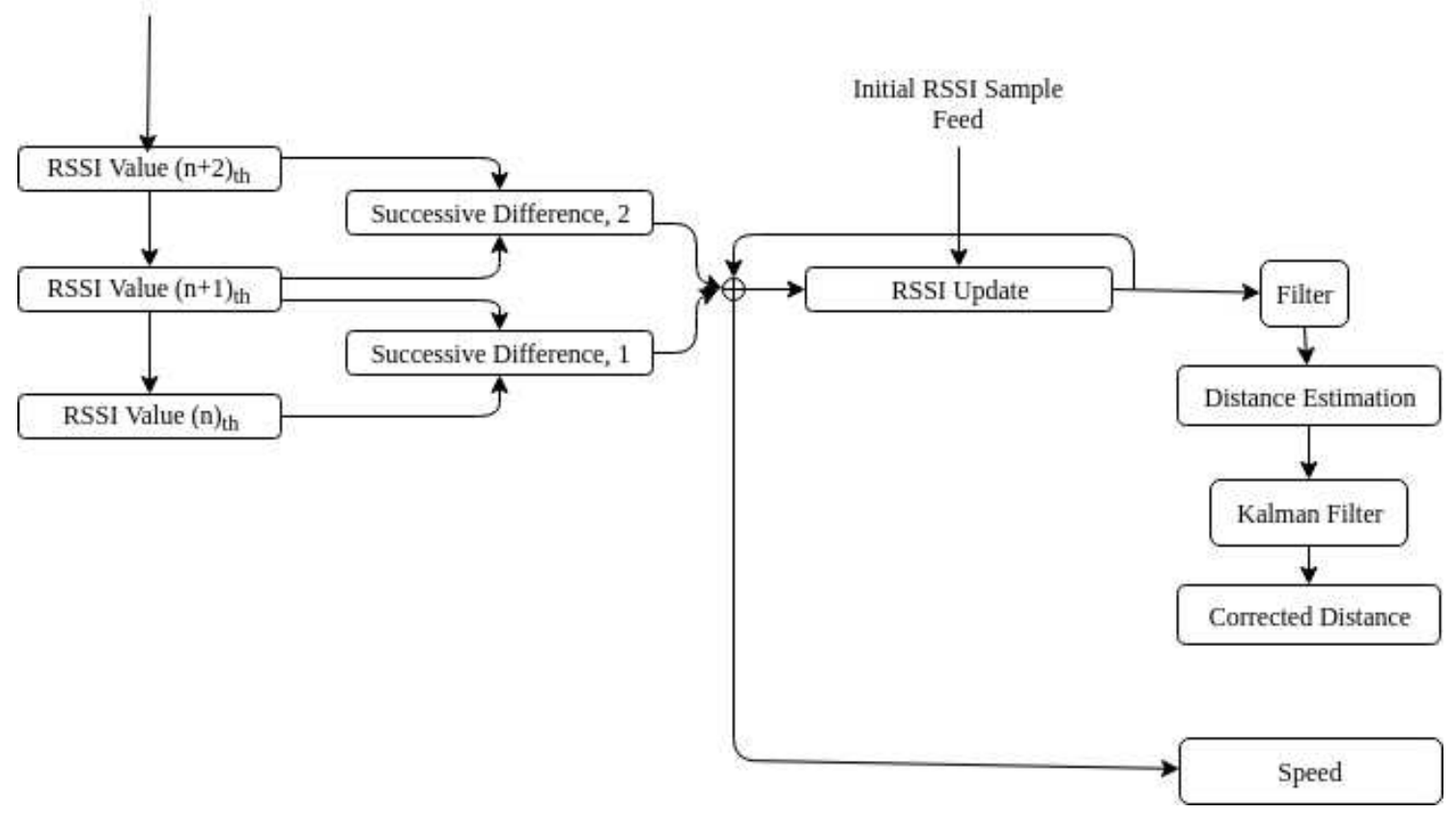

Figure 3.2: Algorithmic flowchart based on Wireless Access Point

\subsubsection{RSSI receiving and Calibration method}

As shown in the figure 3.2, initial RSSI feed at one meter distance of client form the WAP is used for calibration. In this, large sample of RSSI feed is recorded for the purpose of calibration and to estimate the finite value of RSSI at one meter distance, is calculated based on Average and Standard deviation of the samples. Large samples of RSSI values are filtered by eliminating the lowest $5 \%$ and highest $5 \%$ of samples having least probability, $p(x) \leq 0.1$.

In real time mode, distance estimation of client is based on instantaneous RSSI and previous four values of RSSI, so that the error due to immediate large change of RSSI can be corrected.

$$
R S S I(d B m)=-10 n \log _{10}\left(d / d_{0}\right)
$$

(Equation 3.5)

Equation 3.5 represents relation between $\operatorname{RSSI}(\mathrm{dBm})$ and distance $($ meter $s)$, where $d_{0}(m)$ is known distance of object and with $R S S I(\mathrm{dBm})$ known at unknown distance $d(m)$ from the WAP system.

$$
R S S I(d B m)=-10 n \log _{10}(d)+A
$$


Equation 3.5 can be written as Equation 3.6 where, $\mathrm{A}$ is the $R S S I(\mathrm{dBm})$ known at a distance of object at one meter from the system. Further IEEE $802.11 \mathrm{a} / \mathrm{g} / \mathrm{p}$ methodological process based on calibrated RSSI is discussed in section 3.4 and its experimentation is discussed in section 4.4.

\subsection{IEEE 802.11 a/g/p based object Localization with RSSI Calibration}
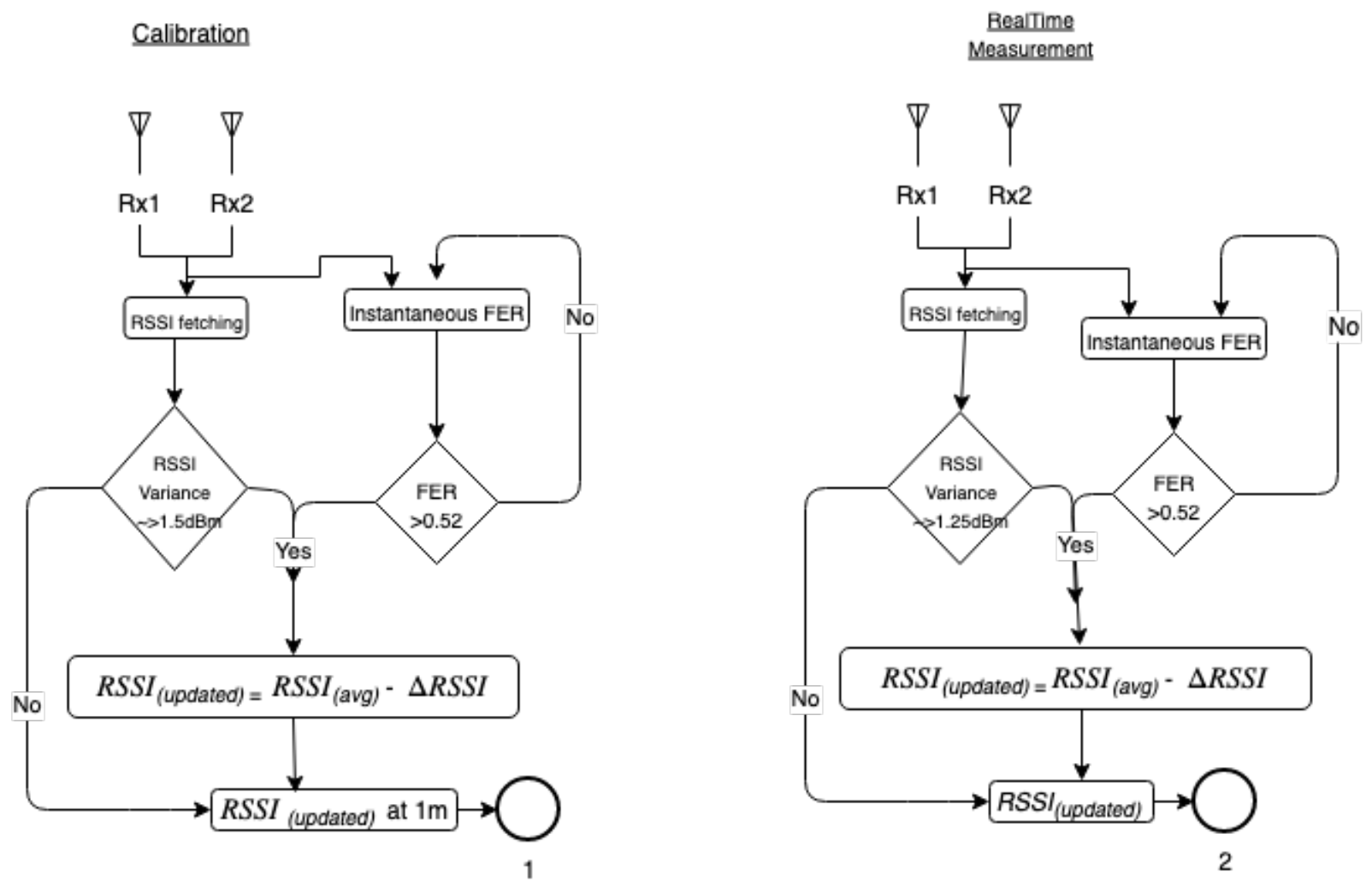

Figure 3.3: Implementation of realtime RSSI receiving and calibration for IEEE $802.11 \mathrm{a} / \mathrm{g} / \mathrm{p}$ localization

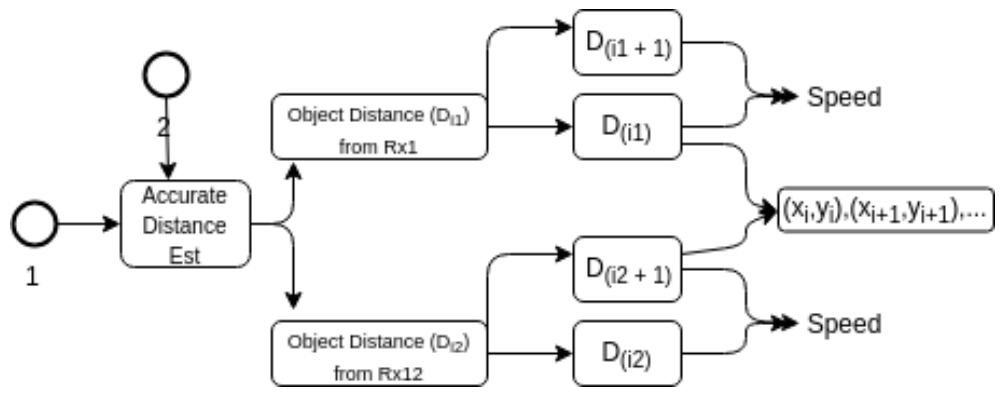

Figure 3.4: Localization Estimation 
As shown in the figure 3.3, initial RSSI feed at one meter distance of client form the IEEE $802.11 \mathrm{a} / \mathrm{g} / \mathrm{p}$ Transceiver is used for calibration. In this, large sample of RSSI feed is recorded for the purpose of calibration and to estimate the finite value of RSSI at one meter distance, is calculated based on Average and Standard deviation of the samples. Large samples of RSSI values are filtered by eliminating the lowest $5 \%$ and highest $5 \%$ of samples having least probability, $p(x) \leq 0.1$.

In realtime processing, as shown in figure 3.3, RSSI feed is instantaneously recorded and calibrated as discussed in further section 3.4.1 based on the instantaneous FER. Its algorithm part for FER based RSSI calibration is shown in section 3.4.1 and experimental result and analysis are discussed in section 4.4. As shown in figure 3.3 consideration of RSSI for $R S S I_{(\text {updated) }}$ at calibration and realtime is based on RSSI variance and FER. In calibration there are large number of samples for finding the best value, it is observed that large variance in RSSI ( approximately greater than $1.5 \mathrm{dBm}$ variance) contributes to the change of approximated RSSI, similarly during realtime measurement approximation of instantaneous RSSI is done by considering instantaneous and last four RSSI values in order to reduce the approximate error, here since the samples are less it is observed that at least variance in RSSI of approx $1.25 \mathrm{dBm}$ has effect on change in RSSI for localization. Moreover the larger value of variance in $R S S I$ leads to FER which in turn increases the probability of change in $R S S I$. During experimentation it is evaluated as shown in table 4.1 that probability, $\left(p_{F E R>0.52}\right)$ of $F E R$ greater than 0.52 is 0.786 which changes the RSSI. 


\subsubsection{FER Calibration and $\Delta R S S I_{\text {avg }}$ Estimation Algorithm}

$R S S I$ and $R S S I_{\text {avg }}$ is calculated for various values of FER, and the change in $R S S I$, i.e $\triangle R S S I$ is estimated for RSSI Calibration and distance estimation.
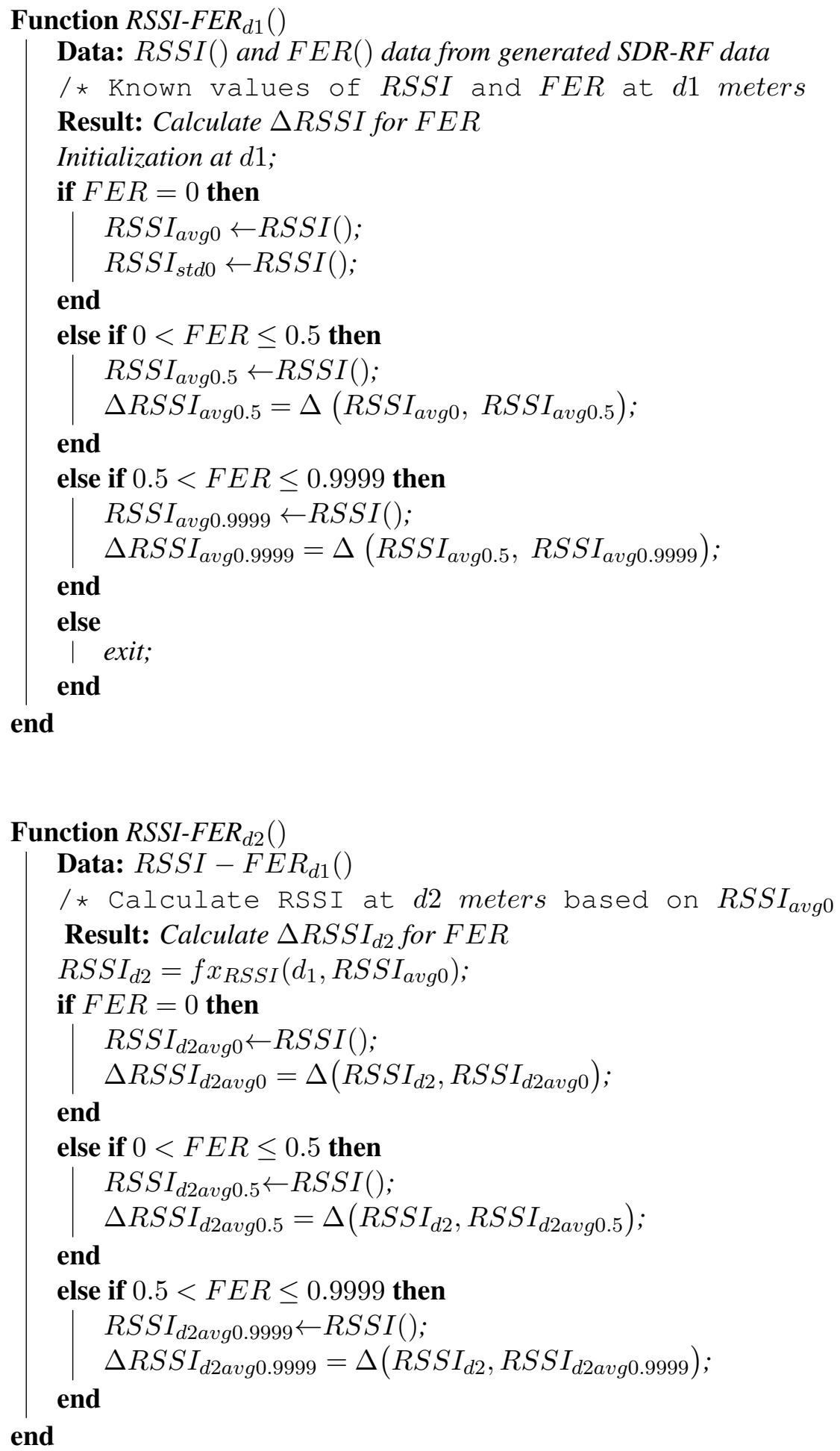
Algorithm as represented through the functions $R S S I-F E R_{d 1}()$ and $R S S I-F E R_{d 2}()$ are used to calculate the change in $R S S I$ (i.e $\triangle R S S I$ ) with change in $F E R$ during calibration and real-time measurements respectively. RSSI() and FER() represents the RSSI and FER data generated through using Software Defined Radio.

In $R S S I-F E R_{d 1}()$ :

- $R S S I()$ is the largest set of $R S S I$ samples generated by SDR at reference distance $d 1$ (meters).

- FER() is the largest set of FER values associated with respective to $R S S I()$ at reference distance $d 1$ (meters).

- $R S S I_{a v g 0}$ is the average value of $R S S I()$ samples at reference distance (d1 meters) for FER= 0 .

- $R S S I_{s t d 0}$ is the standard deviation value of $R S S I()$ samples at the reference distance ( $d 1$ meter $\left.s\right)$ for $F E R=0$.

- $R S S I_{a v g 0.5}$ is the average value of $R S S I()$ at reference distance ( $d 1$ meters) for $0<F E R \leq$ 0.5 .

- $\Delta R S S I_{a v g 0.5}$ is the difference value between $R S S I_{a v g 0}$ and $R S S I_{a v g 0.5}$.

- $R S S I_{a v g 0.9999}$ is the average value of $R S S I()$ at reference distance (d1 meters) for $0.5<F E R$ $\leq 0.9999$.

- $\triangle R S S I_{a v g 0.9999}$ is the difference value between $R S S I_{a v g 0}$ and $R S S I_{a v g 0.9999}$.

\section{At a calibration reference distance of $d_{1}$ as shown in function $R S S I-F E R_{d 1}()$ :}

- Firstly, $R S S I_{a v g 0}$ and $R S S I_{s t d 0}$ is calculated from $R S S I()$ when $F E R=0$. This $R S S I_{\text {avg } 0}$ and $R S S I_{s t d 0}$ are used as a reference values at calibration distance having zero FER.

- Next, if $0<F E R \leq 0.5$ then, $R S S I_{a v g 0.5}$ is calculated from $R S S I()$.

- Here, change in $R S S I$ is calculated when there is a change of $F E R$ from $F E R=0$ to $0<F E R \leq 0.5$. The calculated change is $\triangle R S S I_{\text {avg } 0.5}$ which is used as a reference values at calibration distance having $F E R$ in range $0<F E R \leq 0.5$.

- Finally, if $0.5<F E R \leq 0.9999$, then $R S S I_{a v g 0.9999}$ is calculated from $R S S I()$.

- Here, change in $R S S I$ is calculated when there is a change of $F E R$ from $F E R=0.5$ to $0.5<F E R \leq 0.9999$. The calculated change is $\triangle R S S I_{a v g 0.9999}$ which is used as a reference values at calibration distance having $F E R$ in range $0.5<F E R \leq 0.9999$. 
In $R S S I-F E R_{d 2}()$ :

- $R S S I_{d 2}$ is the calculated $R S S I$ at $d 2$ (meters) distance using $R S S I_{a v g 0}$ obtained at $d 1$ reference distance.

- $R S S I()$ ( when considered in $R S S I-F E R_{d 2}()$ ) is the largest set of $R S S I$ samples generated by SDR at real-time distance, $d 2$ (meters).

- $\triangle R S S I_{d 2 a v g 0}$ is the average value of $R S S I$ samples generated at $d 2$ meters for $F E R=0$.

- $\triangle R S S I_{d 2 a v g 0.5}$ is the average value of $R S S I$ samples generated at $d 2$ meters for $0<F E R \leq$ 0.5 .

- $\triangle R S S I_{d 2 a v g 0.9999}$ is the average value of $R S S I$ samples generated at $d 2$ meters for $0<F E R \leq$ 0.9999 .

- $\triangle R S S I_{d 2 a v g 0.5}$ is the difference value between $R S S I_{d 2}$ and $R S S I_{d 2 a v g}$ for $F E R=0$.

- $\triangle R S S I_{d 2 a v g 0.5}$ is the difference value between $R S S I_{d 2}$ and $R S S I_{d 2 a v g}$ for $0<F E R \leq 0.5$.

- $\triangle R S S I_{d 2 a v g 0.9999}$ is the difference value between $R S S I_{d 2}$ and $R S S I_{d 2 a v g}$ for $0.5<F E R \leq$ 0.9999 .

At the real-time measurement distance of $d_{2}$ as shown in algorithmic function $R S S I-F E R_{d 2}()$ : Calibration data from the function $R S S I-F E R_{d 1}$ and $R S S I()$ during real-time measurement is fetched.

- Firstly, $R S S I$ at $d 2$ meters (i.e $R S S I_{d 2}$ ) is calculated using the values $d 2, d 1$ and calibrated $R S S I_{a v g 0}$ at $d 1$ meters. $f x_{R S S I}\left(d_{1}, R S S I_{\text {avg } 0}\right)$ results the value of $R S S I_{d 2}$.

- Next, if $F E R=0$ then, $\triangle R S S I_{d 2 a v g 0}$ is calculated.

- Here, change in calculated $R S S I$ (i.e $\Delta R S S I_{d 2}$ ) and measured $R S S I$ (i.e, $R S S I_{d 2 a v g 0}$ ) is calculated. The calculated change, $\Delta R S S I_{d 2 a v g 0}$ represents the change in $R S S I$ at zero FER.

- Then, if $0<F E R \leq 0.5$ then, $\Delta R S S I_{d 2 a v g 0.5}$ is calculated.

- Here, change in calculated $R S S I$ (i.e $\Delta R S S I_{d 2}$ ) and measured $R S S I$ (i.e, $R S S I_{d 2 a v g 0.5}$ ) is calculated. The calculated change, $\triangle R S S I_{d 2 a v g 0.5}$ represents the change in $R S S I$ for $F E R$ values in range $0<F E R \leq 0.5$.

- Finally, if $0.5<F E R \leq 0.9999$

- Here, change in calculated $R S S I$ (i.e $\Delta R S S I_{d 2}$ ) and measured $R S S I$ (i.e, $R S S I_{d 2 a v g 0.9999}$ ) is calculated. The calculated change, $\triangle R S S I_{d 2 a v g 0.9999}$ represents the change in $R S S I$ for $F E R$ values in range $0.5<F E R \leq 0.9999$.

Experimentation analysis using the results for section 3.4 are demonstrated and discussed in section 4.4 . 


\subsection{Hardware}

\section{Single-Board Computer}

Single Board computer such as Raspberry pi as shown in Figure 3.5 or BeagleBone Black are low cost microprocessors which has revolutionized the way of using tiny computers for Internet of Things applications especially for basic provision of doing research. Generally these computers have moderate performance and can be used for generating narrow band Rf signals .

The latest version of Raspberry Pi has the following features.

Raspberry Pi 3:

- Quad Core 1.2GHz Broadcom BCM 2837 64bit CPU

- 1GB RAM

- BCMBCM43438 wireless LAN and Bluetooth

- Low Energy (BLE) on board

- 40-pin extended GPIO

- 4 USB 2 ports

- 4 Pole stereo output and composite video port

- Full size HDMI

- CSI camera port for connecting a Raspberry Pi camera

- DSI display port for connecting a Raspberry Pi touchscreen display

- Micro SD port for loading your operating system and storing data

- Upgraded switched Micro USB power source up to 2.5A

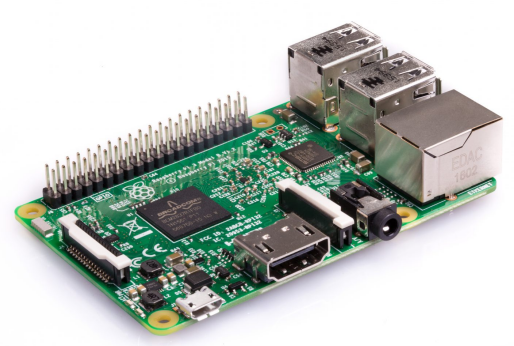

Figure 3.5: Basic Model of Raspberry pi (Raspberry Pi foundation., 2016) 


\section{Software Defined Radio (SDR)}

Software Defined Radio Peripheral is an inexpensive radio frequency transceivers which finds its application in low range radar engineering for chirp generation. ADALM-PLUTO as shown in Figure 3.6 is the basic inexpensive hardware for transceiving wide range of frequencies.

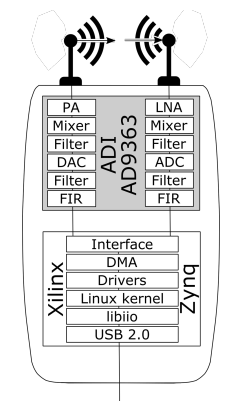

Figure 3.6: ADALM-PLUTO (Analog Devices., 2017)

The following are the features of the ADAM-PLUTO sdr:

- Based on Analog Devices AD9363-Highly Integrated RF Agile Transceiver and Xilinx® Zynq Z-7010 FPGA.

- Radio frequency coverage ranging from $325 \mathrm{MHz}$ to $3.8 \mathrm{GHz}$.

- $20 \mathrm{MHz}$ instantaneous bandwidth.

- 12-bit ADC and DAC.

- Sampling rate upto 61.44 mega samples per second (MSPS)

- One transmitter and one receiver with half or full duplex

- MATLAB, Simulink and GNU Radio sink source block support.

- libiio, a C, C++, C, and Python API.

- Linux, Windows and OS X operating system support.

- USB 2.0 powered interface. 


\subsection{Software}

\section{GNU Radio - SDR software}

GNU Radio [13] is a free and open source software toolkit for signal processing for implementing software defined radios. It has features of simulation environment and also for interfacing hardware for generating and processing signals. GNU Radio along with SDR is used in Raspberry Pi for generation of RF signals.

\section{BASH}

Bash is a command line scripting language. It is a core component of Linux based systems. For users, this is mostly used for automating the system process through series of command execution written in bash. In this thesis, bash scripting is used for automating the system process while performing the experiment.

\section{Python - Programming Language}

Python is a general-purpose language. In this thesis, it is mainly used for performing numerical and scientific computations.

\section{Operating System}

Linux operating system such as Debian, Ubuntu or, Raspbian OS especially for raspberry pi is used . As these distributions are based on Linux kernel which Free and Open source Software giving flexibility of editing the source code and also adding addon's applications. Raspberry pi is mounted with Linux operating System and addon's such as Hostapd.

\subsection{Proposed Model}

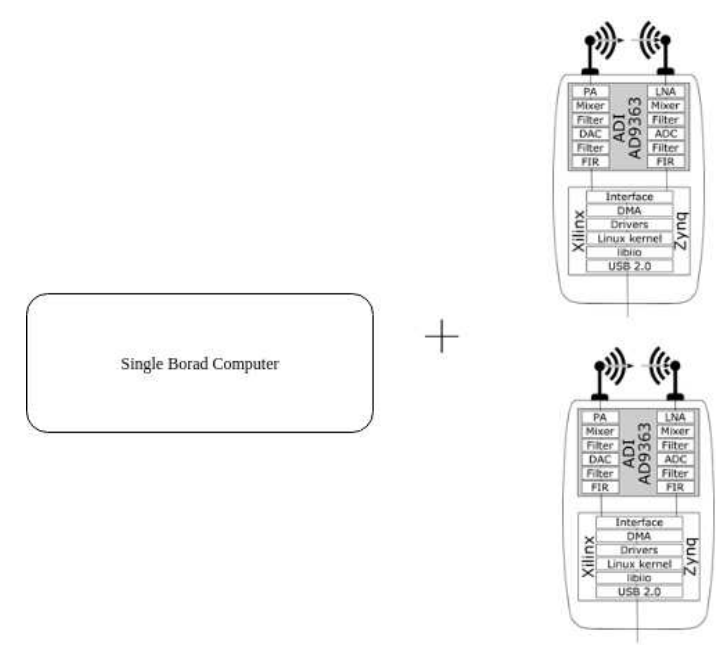

Figure 3.7: Proposed Hardware model 


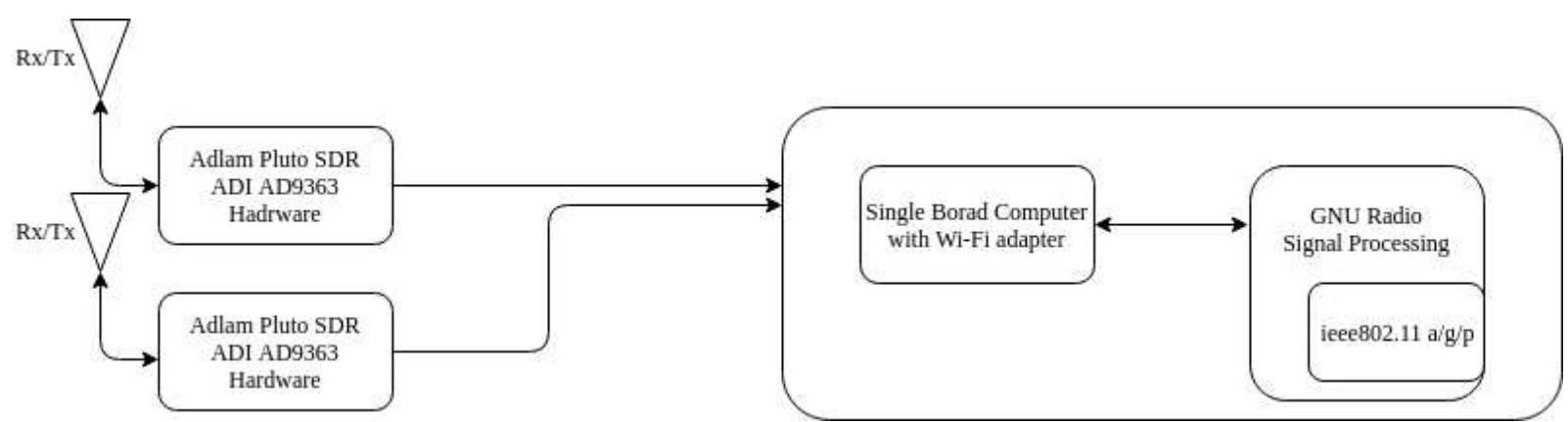

Figure 3.8: Connection diagram for proposed model

Figure 3.7 is the physical realization of proposed model with Raspberry Pi, Pluto SDR and respective connection diagram is shown in figure 3.8 . 


\section{CHAPTER 4}

\section{EXPERIMENTATION RESULTS}

\subsection{Introduction}

In this chapter, experimentation results and analysis are done for Power Analysis in the section 4.2, for Wireless Access Point based localization in the section 4.3 and IEEE $802.11 \mathrm{a} / \mathrm{g} / \mathrm{p}$ RSSI calibration based localization in section 4.4 .

\subsection{Power Analysis}

The following are the experimentation results of Power Analysis methodology as discussed in section 3.2 of chapter 3.

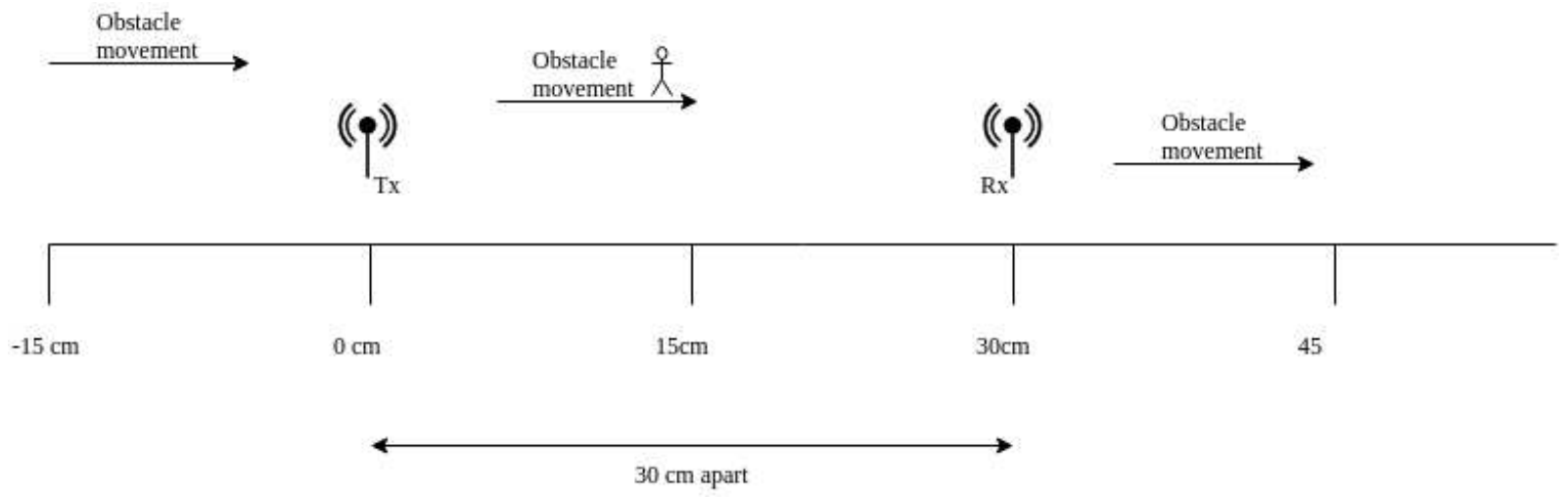

Figure 4.1: Power Analysis Experimentation Scenario

Experimentation results are performed as describe in the following:

- Obstacle is introduced at different distance (LOS), moving in range from left of transmitter(Tx) to the receivers $(R x)$ right scaling from $-15 \mathrm{~cm}$ to $45 \mathrm{~cm}$ as shown in Figure 4.1 and observations are recorded.

- Experiment is repeated for three times (at different timings)with a stamp for every $3 \mathrm{~cm}$.

- For each time and for every stamp of 3cm, 6 values are observed and average are taken into consideration for every graph analysis. 


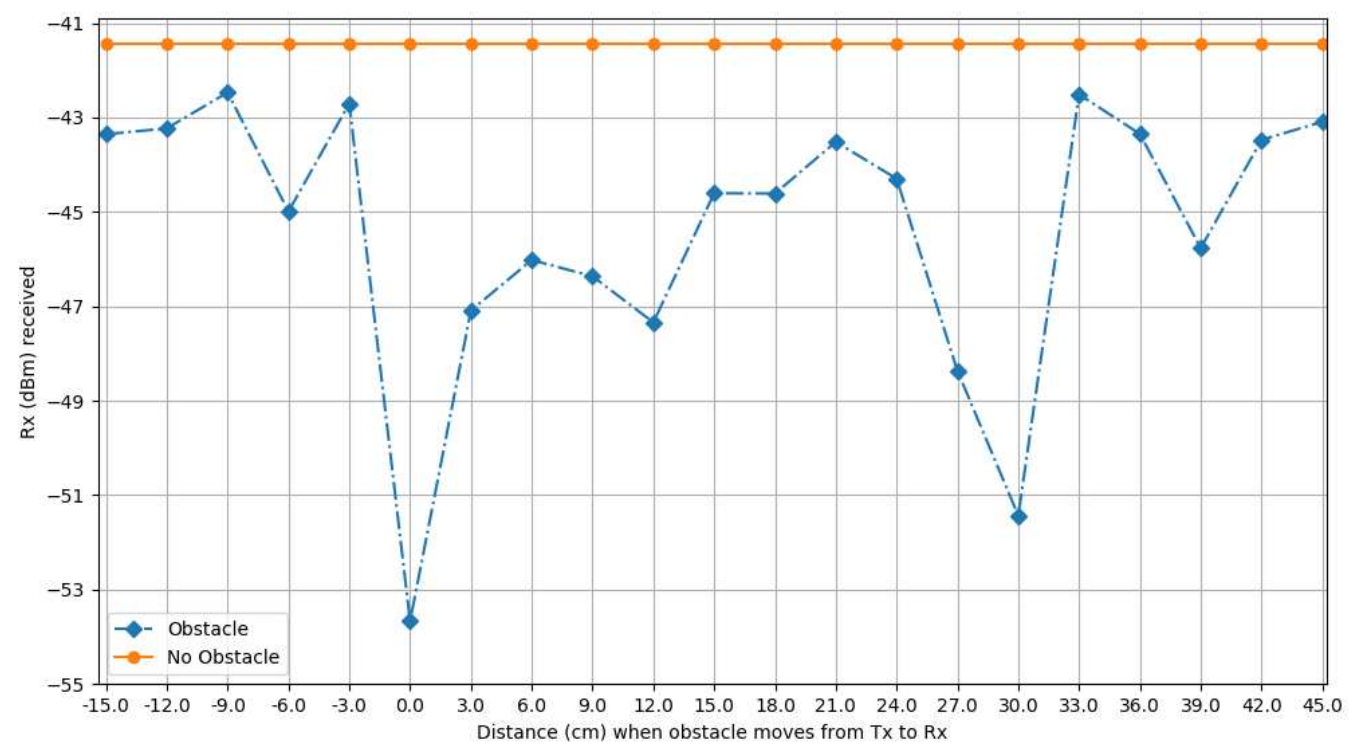

Figure 4.2: Power Analysis Experimentation 1

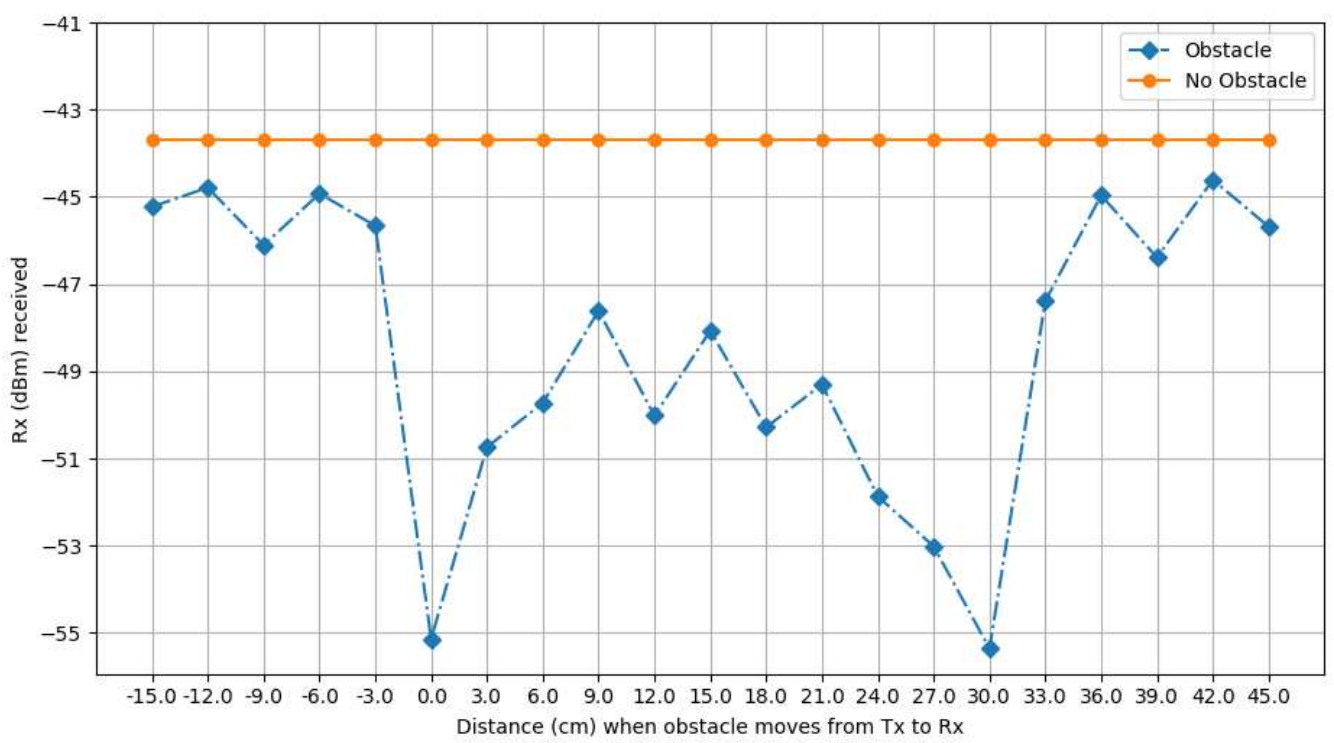

Figure 4.3: Power Analysis Experimentation 2 


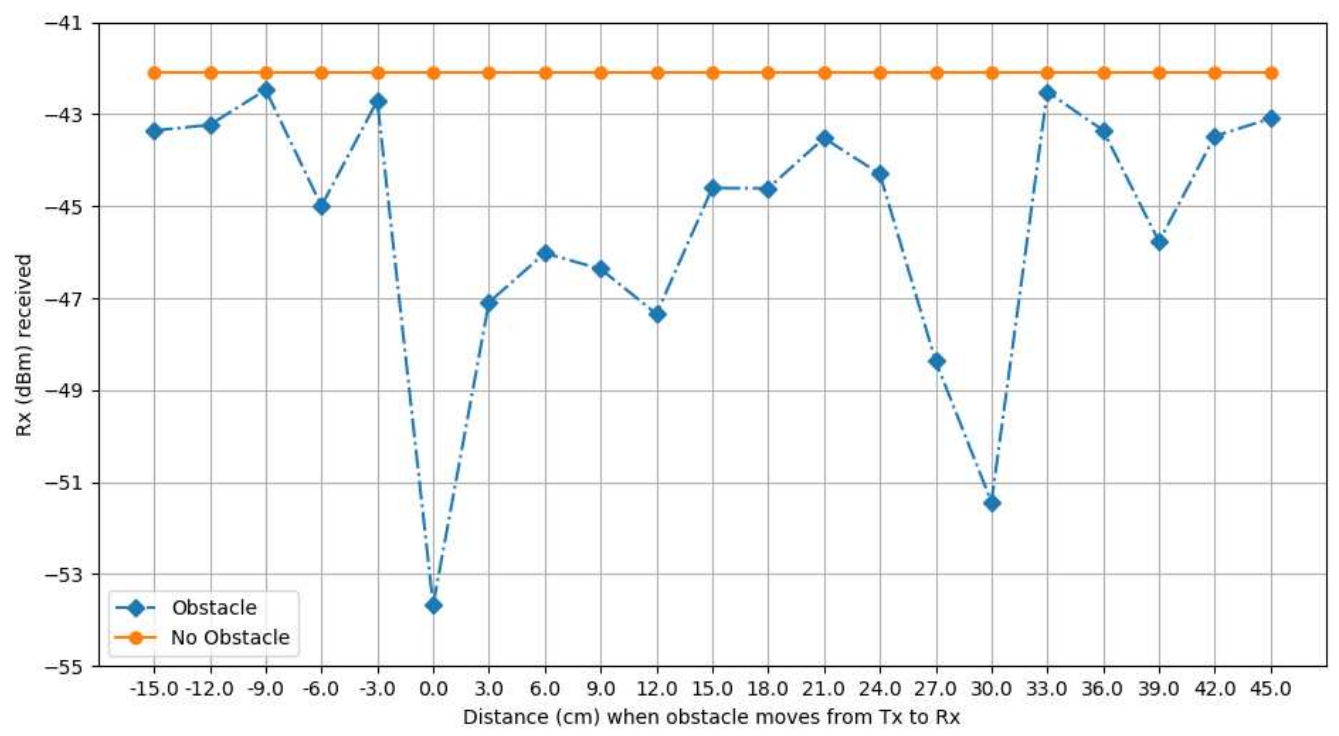

Figure 4.4: Power Analysis Experimentation 3

Power Analysis: Graph Analysis: Graphs represented through figure 4.2, figure 4.3 and figure 4.4 is a graphical signal representation of experimentation scenario in figure 4.1. These three graphs (figures 4.2, 4.3 and 4.4 ) are generated using the same methodology for the scenario in figure 4.1, but the experimentation is performed at different time. Purpose of this to lay and analogy for using power analysis based experimentation for localization, which is discussed in the following sections.

For analysis we divide the scenario into four parts such as left of $T x(-15 \mathrm{~cm}$ to $0 \mathrm{~cm})$, right of $T x(0 \mathrm{~cm}$ to $15 \mathrm{~cm})$, left of $R x(15 \mathrm{~cm}$ to $30 \mathrm{~cm})$ and right of $T x(30 \mathrm{~cm}$ to $45 \mathrm{~cm})$.

- Right of $T x(0 \mathbf{~ c m}$ to $15 \mathbf{~ c m})$ : $R x d B m$ increases till the mid value of right of $T x(0 \mathrm{~cm}$ to 15 $\mathrm{cm})$, about $7.5 \mathrm{~cm}$ then the graph starts decreases from that midpoint to endpoint of right of $\operatorname{Tx}(0$ $\mathrm{cm}$ to $15 \mathrm{~cm}$ ), i.e mid point of $T x-R x$ distance.

Here the effect of small sidelobes are reduced till the quarter distance of $30 \mathrm{~cm}$ due to which the graph increases and in the next quarter often sidelobes effect is not nullified and the signal reflection from obstacle can cause a decrease of signal level due to which graph falls down.

- Left of $R x(15 \mathrm{~cm}$ to $30 \mathrm{~cm})$ : from mid point of $T x-R x$, graph starts increasing till the mid point of left of $R x(15 \mathrm{~cm}$ to $30 \mathrm{~cm})$, about at $21 \mathrm{~cm}$ and then the graphs start decreasing until the end point of left of $R x(15 \mathrm{~cm}$ to $30 \mathrm{~cm})$, i.e at $30 \mathrm{~cm}$ near the receiver. The value of received $d B m$ is low at $30 \mathrm{~cm}$ point because of blockage of the signal from the transmitter $(T x)$ to the receiver $(R x)$ by the obstacle.

- Left of $T x(\mathbf{- 1 5} \mathbf{~ c m}$ to $\mathbf{0 ~} \mathbf{~ c m})$ : Similar effect, Transmitted power level is less when obstacle is at $T x(0 \mathrm{~cm})$ and it increases as the obstacle moves from farther left of $T x$ and starts decreasing from 
the quarter point $(0 \mathrm{~cm}$ to $-15 \mathrm{~cm})$ i.e the effect of side lobe is observed and it starts increasing from point the mid point when the sidelobe effect is reduced. And then the reflections from the main lobe due to the obstacle can cause the signal to interference and the signal attenuation is observed at the receiver.

- Right of $R x(30 \mathrm{~cm}$ to $45 \mathrm{~cm})$ : Similar effect of sidelobe and main lobe reflection by the obstacle when it moves from right to farther right of $R x$ can cause increase and decrease of signal power.

Ratio of Average received signal in $d B m$ when there is no presence of obstacle to the received signal $d B m$ when obstacle is introduced at instantaneous distance from $T x$ is approximately equal for all the experiments shown in graphs represented through figure 4.2, figure 4.3 and figure 4.4

$$
\text { Power AnalysisExperiment }\left(\frac{\text { Average } R x d B m \text { received when no obstacle }}{R x d B m \text { received instantaneously when obstacle is present }}\right)
$$

(Equation 4.1)

From the graphical analysis represented through figure 4.2 , figure 4.3 and figure 4.4 , an inference can be laid that the instantaneous value of the equation 4.1 is approximately equal for all the three experiments (as represented through figure 4.2, figure 4.3 and figure 4.4 ). 


\subsection{Wireless Access Point Localization}

In general, the proposed system consist of Raspberry Pi (as Access Point) and a Software Defined Radio which is used for object localization. Here as Raspberry pi is used as an Access Point. This can be used as single Access Point localization along with the object localization with the Two receivers and transmitters of SDR's which can increase the accuracy of detection. The following section performs the experimentation in finding the distance of object from the Raspberry Pi Access point.

\subsubsection{Experimentation for distance determination}

After the client is associate with the Access Point. RSSI values of the client at the Access Point is obtained and the distance calculation is done. This experimentation tells that how accurate that the single Wi-Fi access point with single antenna is.

- The readings of the RSSI received at the AP is read by AP, by placing the Mobile client (connected to AP) at varies distances ranging from one meter to five meters with an interval of one meter. This experimentation is performed twice. Average RSSI values are calculated and the distance estimation is done in the later section $\mathrm{s}$ of the documents. The experimentation scenario is observed in the figure 4.5

- Again Now the AP is placed at the center and the Mobile client is made to move from left of AP and also to Right of AP as shown in figure 4.6

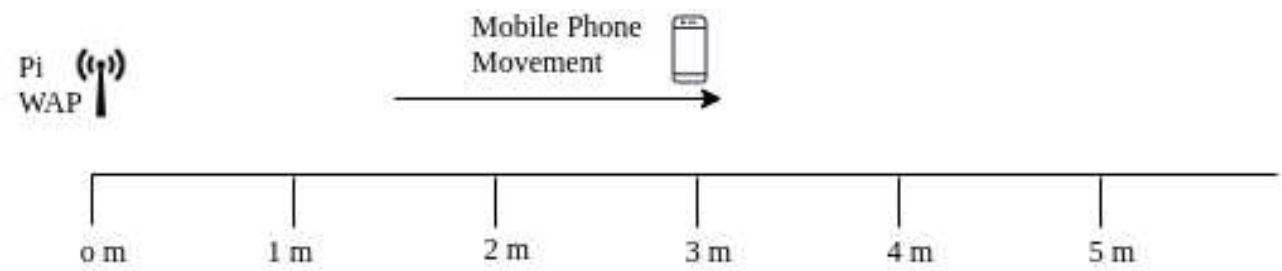

Figure 4.5: Experiment, when Mobile client is moving from Access Point

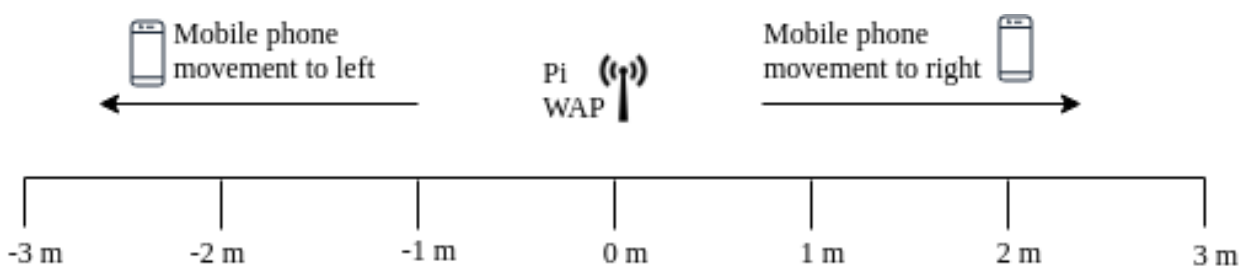

Figure 4.6: Experiment, When mobile client is moving from left and right of AP 


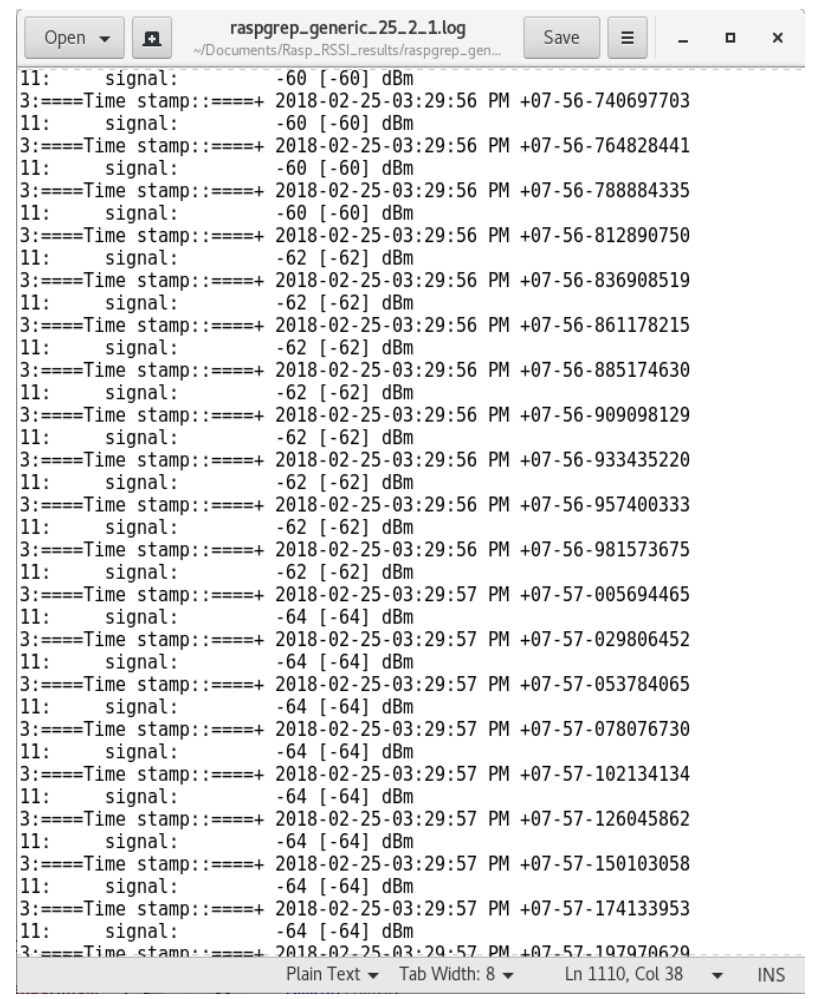

Figure 4.7: RSSI reception by using bash shell scripting

We know that RSSI to the distance conversion is as shown in the Equation 4.2. Graphs in Figures 4.8, 4.9, 4.10 and 4.11 are plotted by showing how an observed(calculated) distance value is relative to the ground truth.

$$
R S S I(d B m)=-10 n \log _{10}\left(d / d_{0}\right)
$$

where $d_{0}(m)$ is known distance of object and with $R S S I$ known at unknown distance of $d$ meters. Equation 4.2 can be written as Equation Equation 4.3 where, $A$ is the $R S S I(d B m)$ known at a distance of object at $1 \mathrm{~m}$ from the system.

$$
R S S I(d B m)=-10 n \log _{10}(d)+A
$$

Distance calculation is done using Equation 4.3 at varies values of path loss component $(n)$ as as described in scenario figure 4.5 and figure 4.6 . 
Following graphs represented through figure 4.8, figure 4.9, figure 4.10 and figure 4.11 are the graphs for the varies values of path loss component $n$.

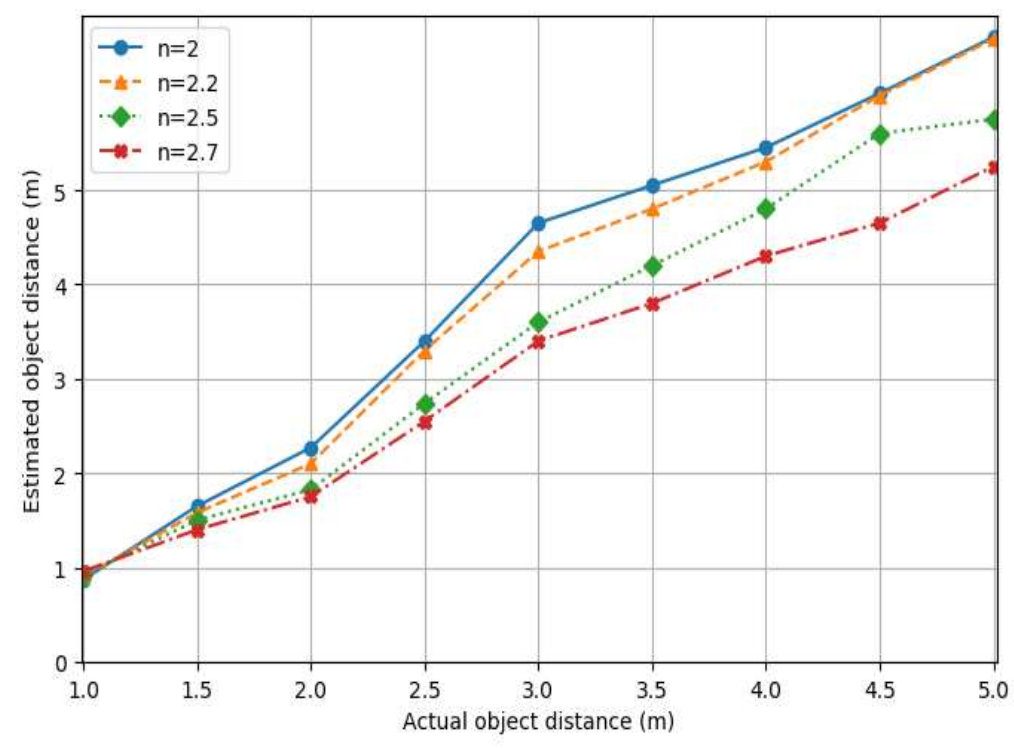

Figure 4.8: Actual distance vs. estimated distance for every one-meter range from experiment 1

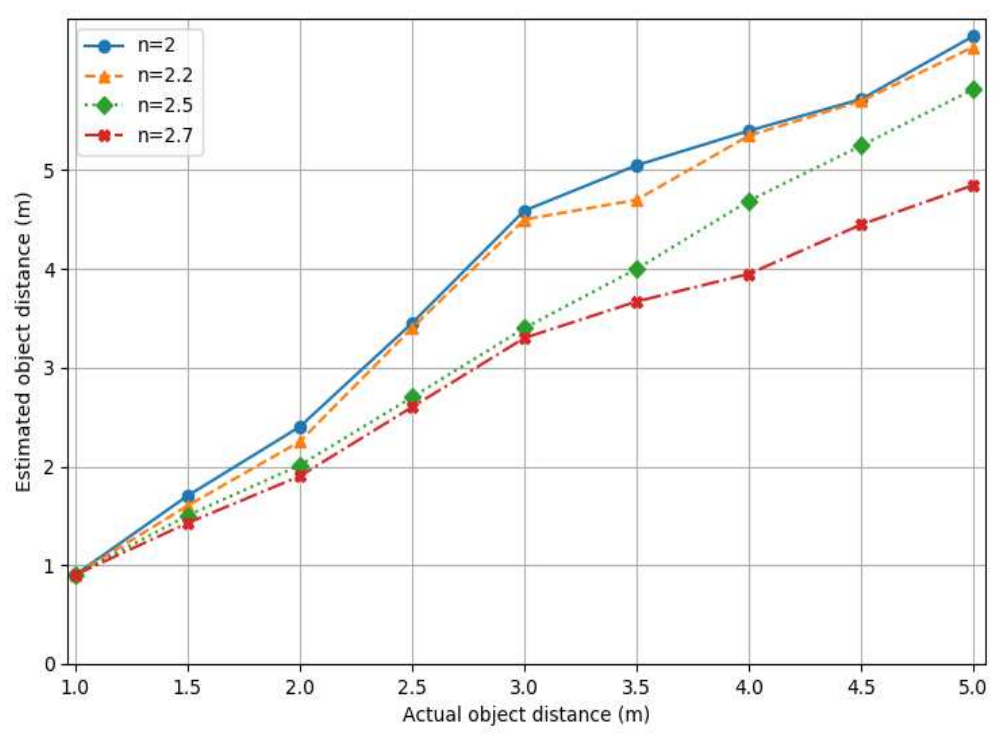

Figure 4.9: Actual distance vs. estimated distance for every one-meter range from experiment 2

The following Figures 4.10 and 4.11 represents how the estimated and the actual ground truth distances 
are varied from the mobile client to the Access Point from different directions.

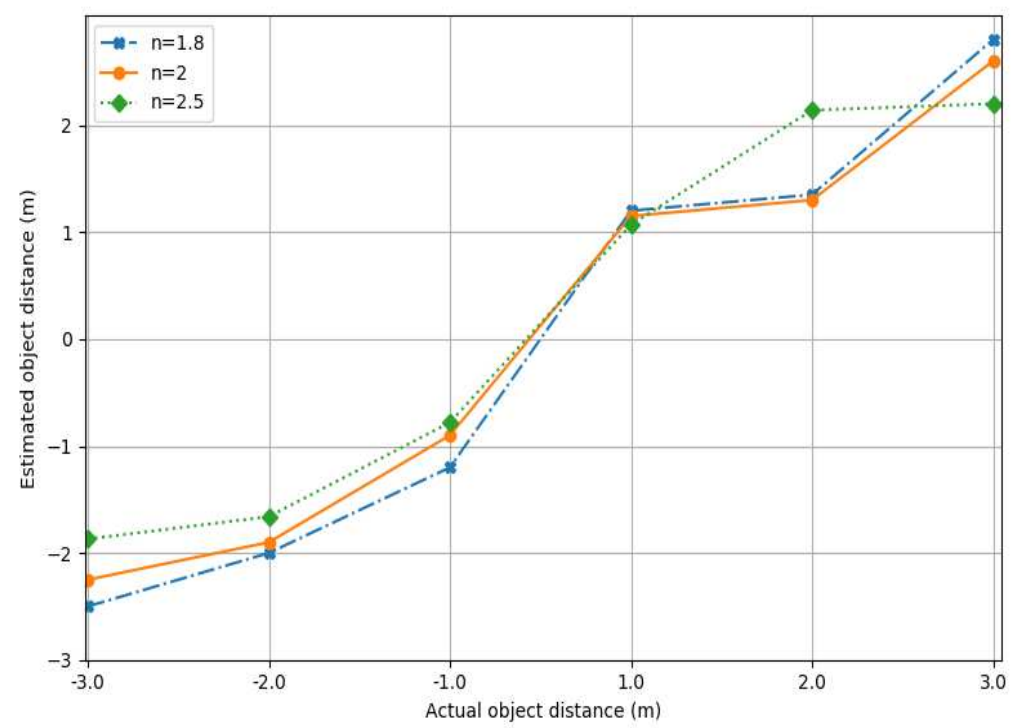

Figure 4.10: Actual distance vs. estimated distance for every one-meter range to the left and right of the AP from experiment 1

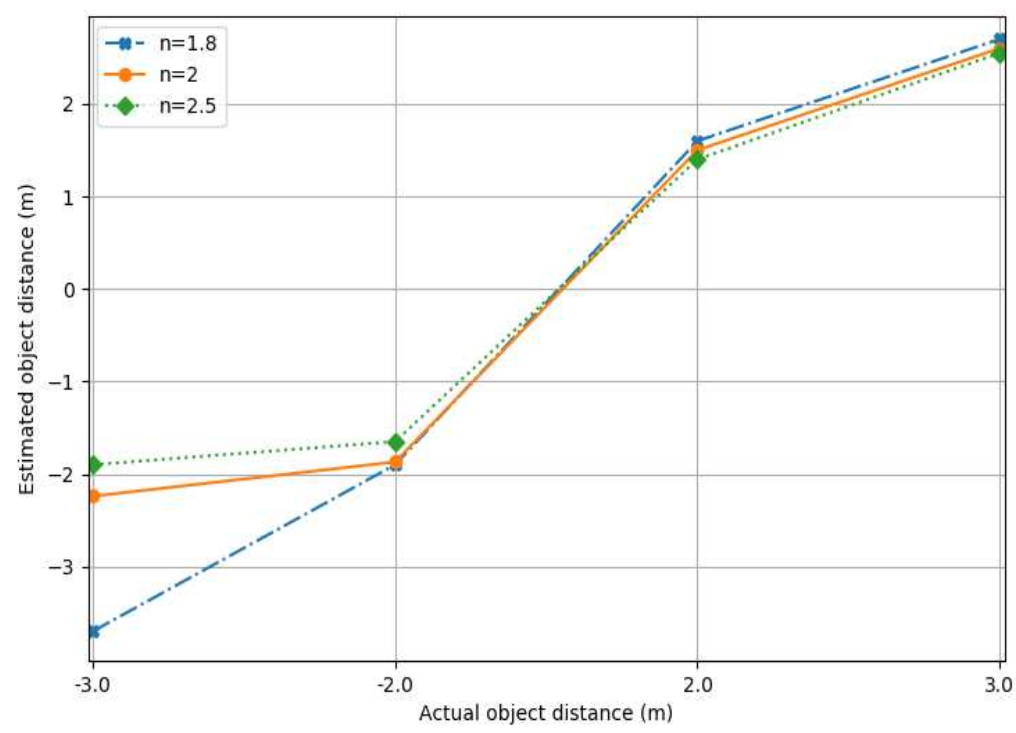

Figure 4.11: Actual distance vs. estimated distance for every one-meter range to the left and right of the AP from experiment 2 

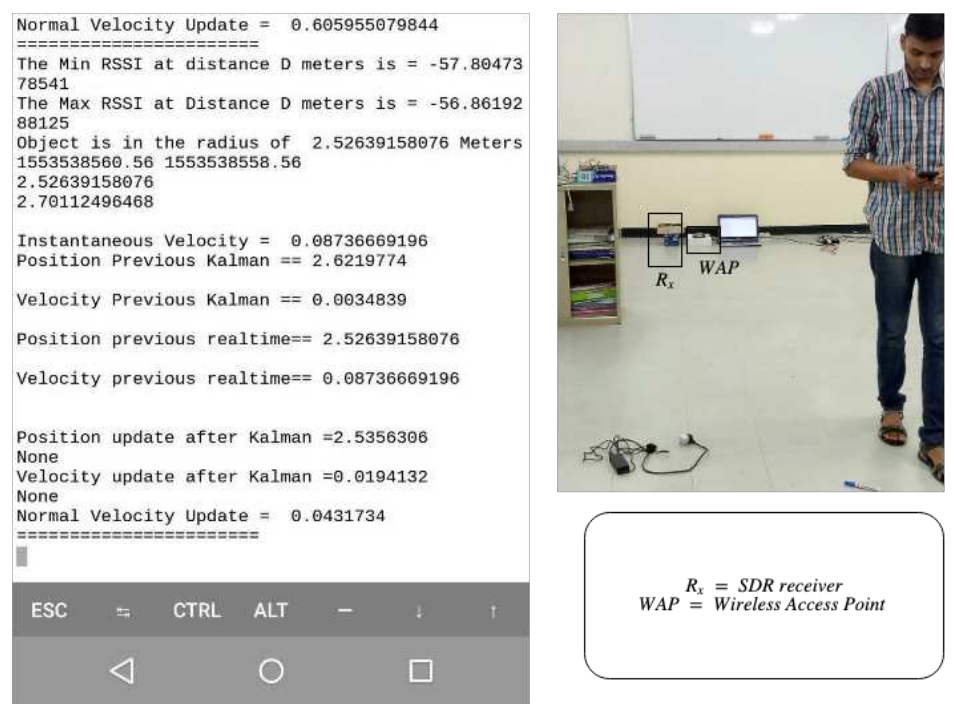

Figure 4.12: Real-time values of WAP based localization on dashboard

Graphs shown in figure 4.13 and 4.14 represents the real time dynamic object localization and its Kalman filtered output for both distance(meters) and velocity(meters/sec). Figure 4.7 and figure 4.12 shows the real-time values of WAP based localization on dashboard, where the scripts are written in for the purpose, in Python and Linux Bash Shell for system automation.

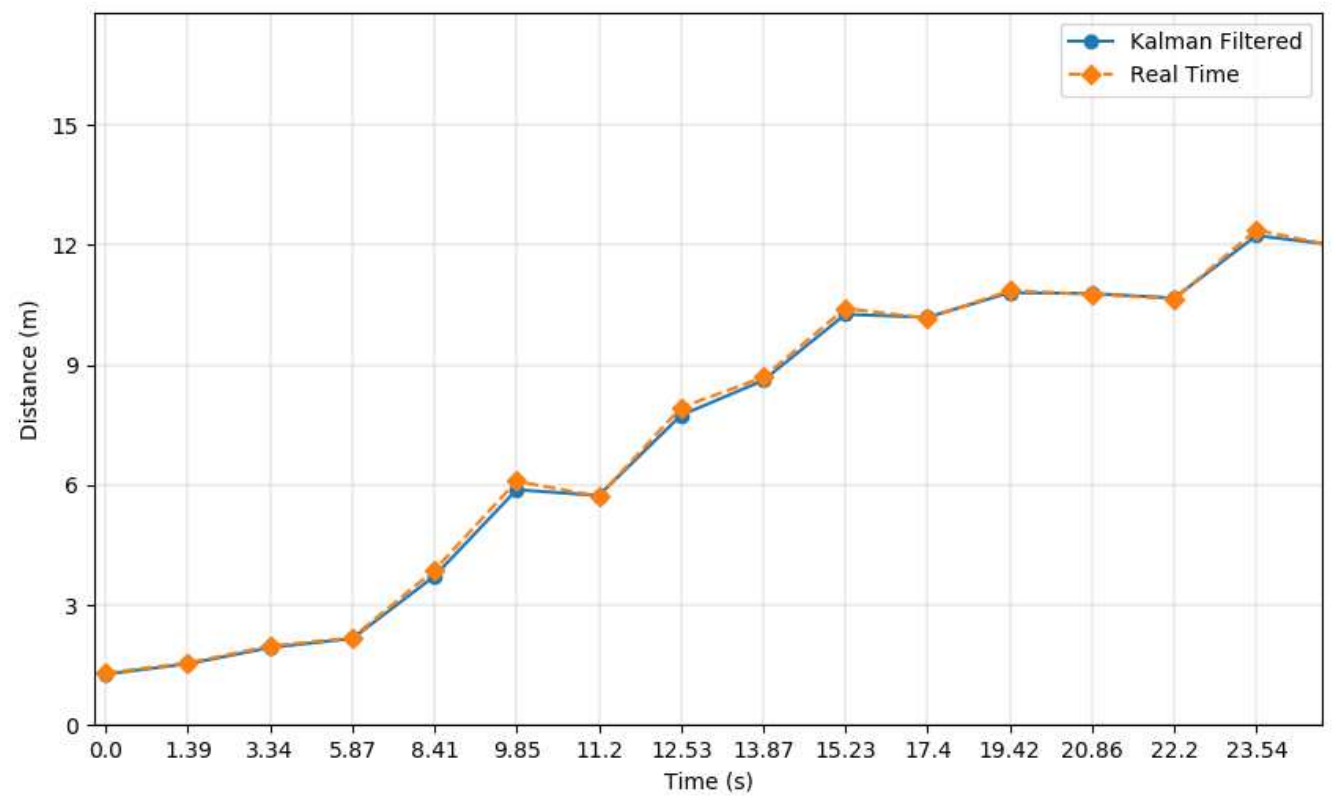

Figure 4.13: Kalman filtered distance and real-time distance 


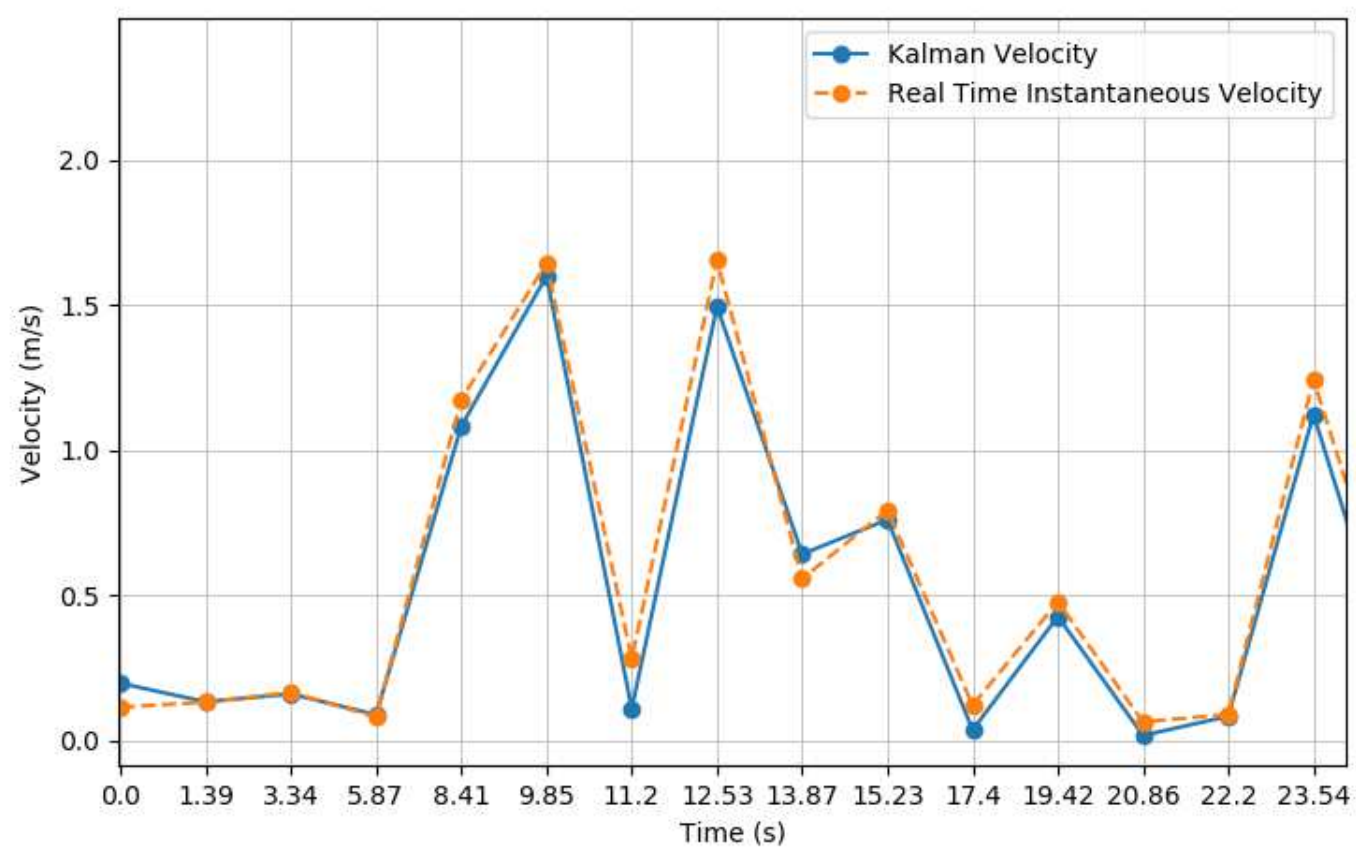

Figure 4.14: Kalman filtered velocity and real-time velocity

The above results shows that distance estimation by using RSSI received at single Access Point. But the accuracy can be increased by using SDR for localizing IEEE $802.11 \mathrm{a} / \mathrm{g} / \mathrm{p}$ based Transceiving devices.

As the proposed system contains Access Point and SDR can accurately detect the object type and can locate them accurately. 


\subsection{RSSI Based on IEEE 802.11 a/g/p implementation}

RSSI based transceiving is implemented using IEEE $802.11 \mathrm{a} / \mathrm{g} / \mathrm{p}$ implementation on Adlam Pluto SDR as demonstrated in the section 3.4 of chapter 3. In this, Instantaneous RSSI through frame receiving is done by the Software Defined Radio receiver and Frame Error Rate (FER) estimation is done. Then analysis of Frame Error Rate (FER) is done and conclusions are drawn for accurate estimation of RSSI for localization with MAC address of object.

\subsubsection{Observed FER and its Analysis}

For the purpose of FER analysis, a large number of RSSI samples and their associated FER values are recorded. This is done by using IEEE $802.11 \mathrm{a} / \mathrm{g} / \mathrm{p}$ based receivers and transmitters at various indoor environments with the similar setups as showing the figure 4.23. This experimentation is performed at different time intervals to approximate the standardized FER for all possible indoor positioning environments.

Following graph shown in figure 4.15 is a density analysis of normalized count of RSSI samples (of about two thousand in number) for various Frame Error Rate (FER). Density analysis shows that most of the samples have FER range in between 0.5 and 0.9999 .

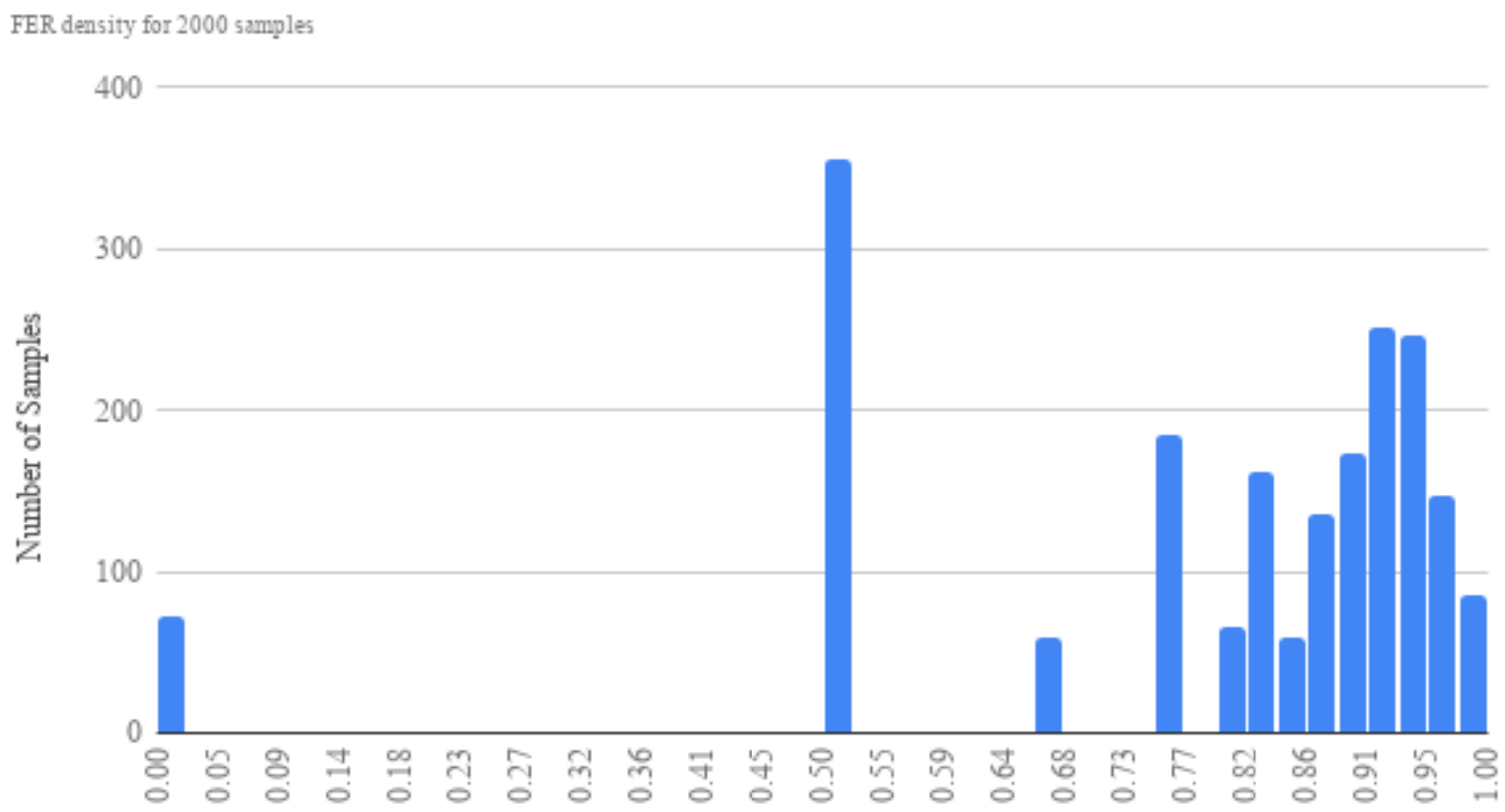

Frame Error Rate

Figure 4.15: Frame Error Rate Analysis 


\subsubsection{Analysis based on FER Probability}

Table 4.1: Probability of FER Occurrence

\begin{tabular}{|l|c|}
\hline FER Probability $(p)$ & Value \\
\hline$p_{(0<=F E R<0.02)}$ & $=0.0360$ \\
\hline$p_{(0.02<=F E R<0.50)}$ & $=0.0000$ \\
\hline$p_{(0.50<=F E R<0.52)}$ & $=0.1780$ \\
\hline$p_{(0.52<=F E R<0.66)}$ & $=0.0000$ \\
\hline$p_{(0.66<=F E R<0.68)}$ & $=0.0295$ \\
\hline$p_{(0.68<=F E R<0.75)}$ & $=0.0000$ \\
\hline$p_{(0.75<=F E R<0.89)}$ & $=0.2745$ \\
\hline$p_{(0.89<=F E R<0.98)}$ & $=0.4820$ \\
\hline
\end{tabular}

From the table 4.1, as shown, an inference can be laid down as shown in figure 4.2.

Table 4.2: Probability of FER Occurrence

$$
\begin{aligned}
& p_{(F E R=0.00)}=0.0360 \\
& p_{(F E R<0.50)}=0.0360 \\
& p_{(F E R>=0.75)}=0.7565
\end{aligned}
$$

Figure 4.2 shows that probability of occurrence of FER being greater than 0.75 is 0.7565 . In the subsequent steps of this section more analysis is done to lay conclusion on FER associated with RSSI. 


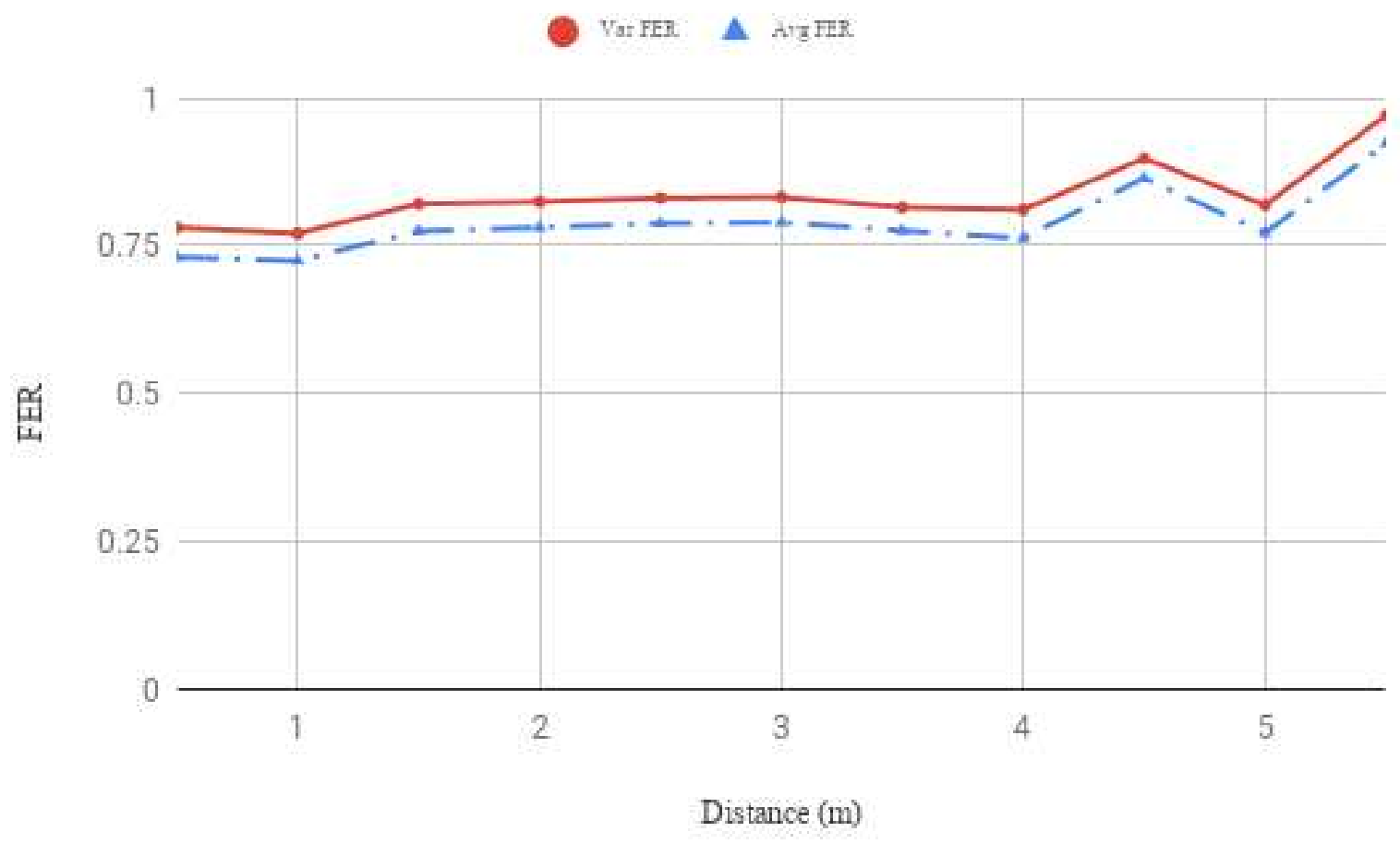

Figure 4.16: FER and Distance 


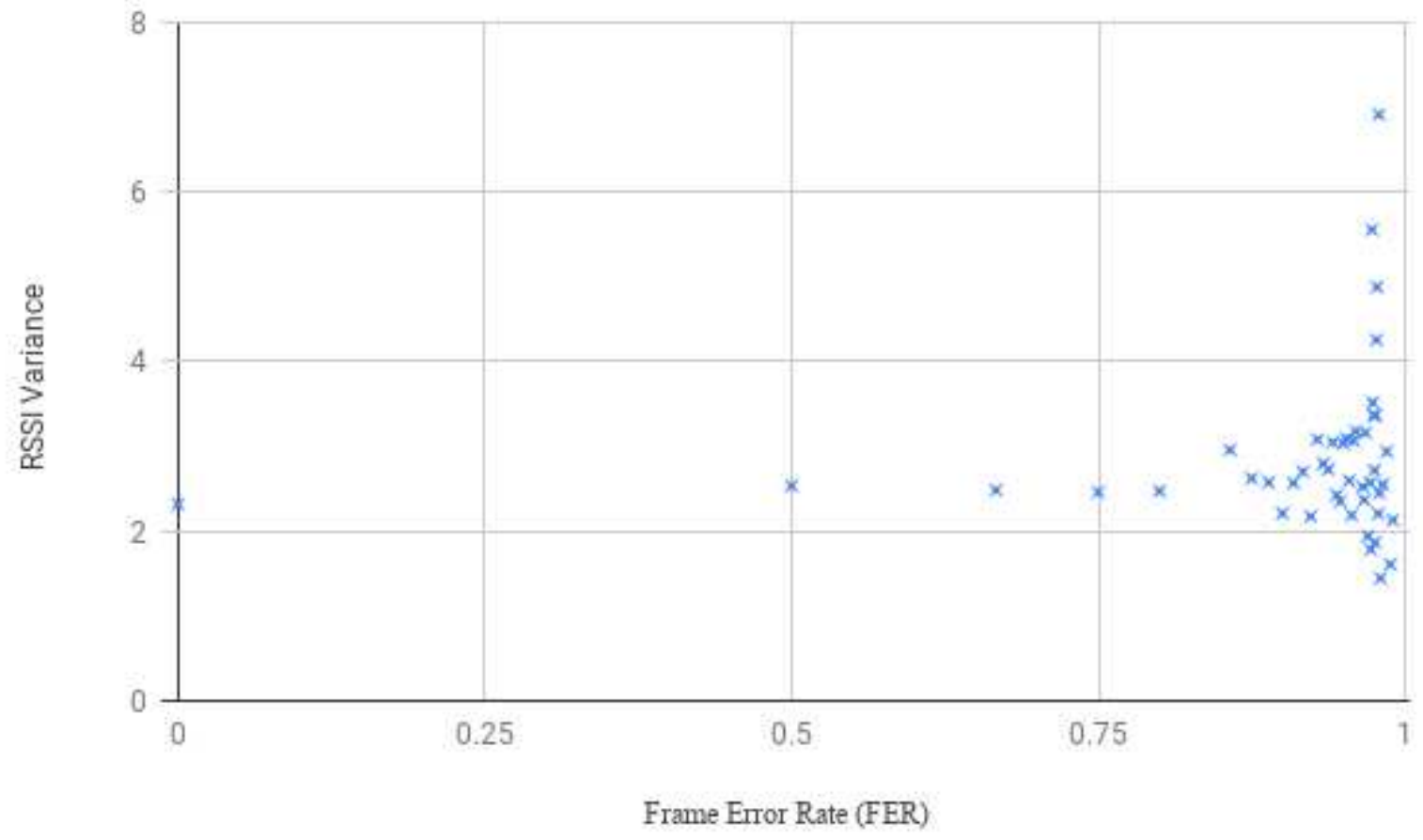

Figure 4.17: FER vs. RSSI Variance

Graph shown in figure 4.16 represents varying Variance of FER with Average FER and graph shown in figure 4.17 represents varying Variance of RSSI with respective to FER. As observed there is a huge density of RSSI variance for the FER in range 0.75 - 0.985 


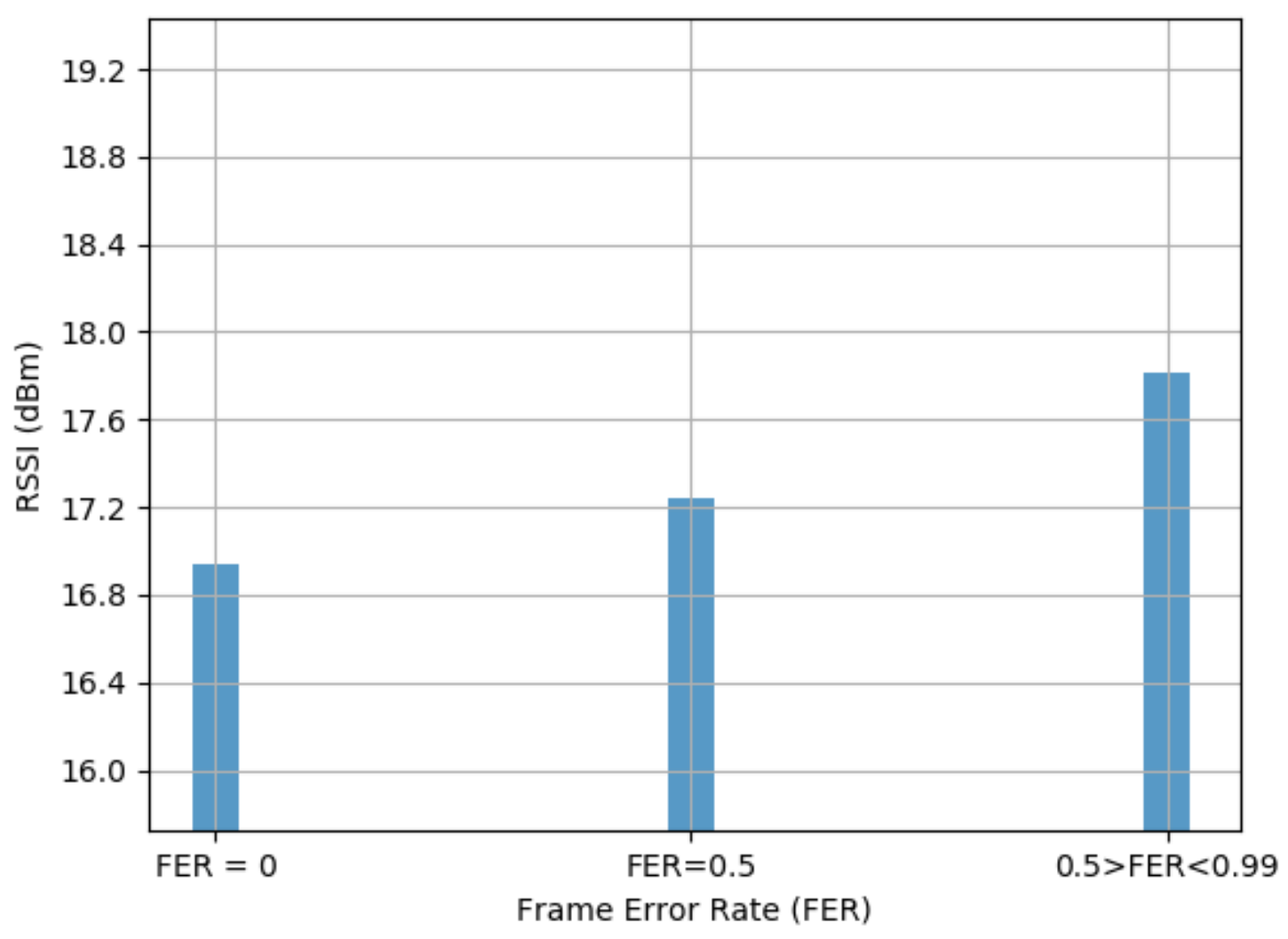

Figure 4.18: FER vs. RSSI

Table 4.3: $\Delta$ RSSI Calibration Values

\begin{tabular}{|l|l|l|l|l|l|}
\hline \multicolumn{7}{|c|}{$\Delta$ RSSIChangeCalculation } \\
\hline 0 & 0.5 & $>0.5$ & $\begin{array}{l}\Delta \text { RSSI } \\
(0,0.5)\end{array}$ & $\begin{array}{l}\Delta \text { RSSI } \\
(0,>0.5)\end{array}$ & $\begin{array}{l}\Delta \text { RSSI } \\
\end{array}$ \\
& & & $0.5,>0.5)$ \\
\hline 16.94134078 & 17.23733984 & 17.81143611 & 0.29599906 & 0.87009533 & 0.57409627 \\
\hline
\end{tabular}




\subsubsection{Distance Estimation based with and without FER based Correction}

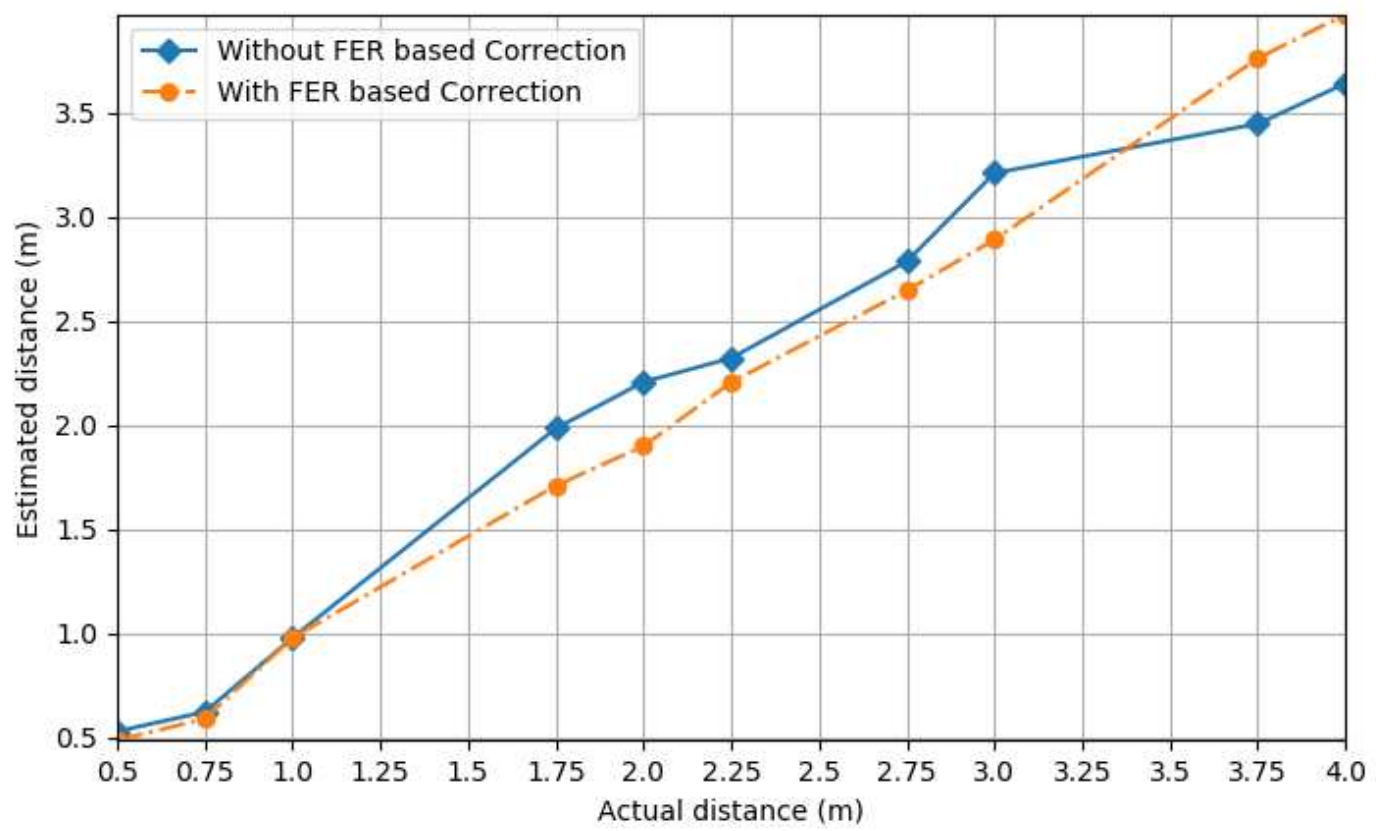

Figure 4.19: Actual distance vs. estimated distance with and without $F E R$ based correction

As described in algorithm in the section 3.4.1, graphs shown in figure 4.18 represents change of RSSI (consolidated representation of values at reference distance) with at various FER. From this an inference as shown in table 4.3 is drawn to calculate $\triangle R S S I$ for $R S S I$ calibration with $R S S I$ change of 0.870 $d B m \approx 1 \mathrm{dBm}$. Graph as shown in figure 4.19 represents evaluated distance $v / s$ actual distance with $F E R$ based correction and without FER based correction. 


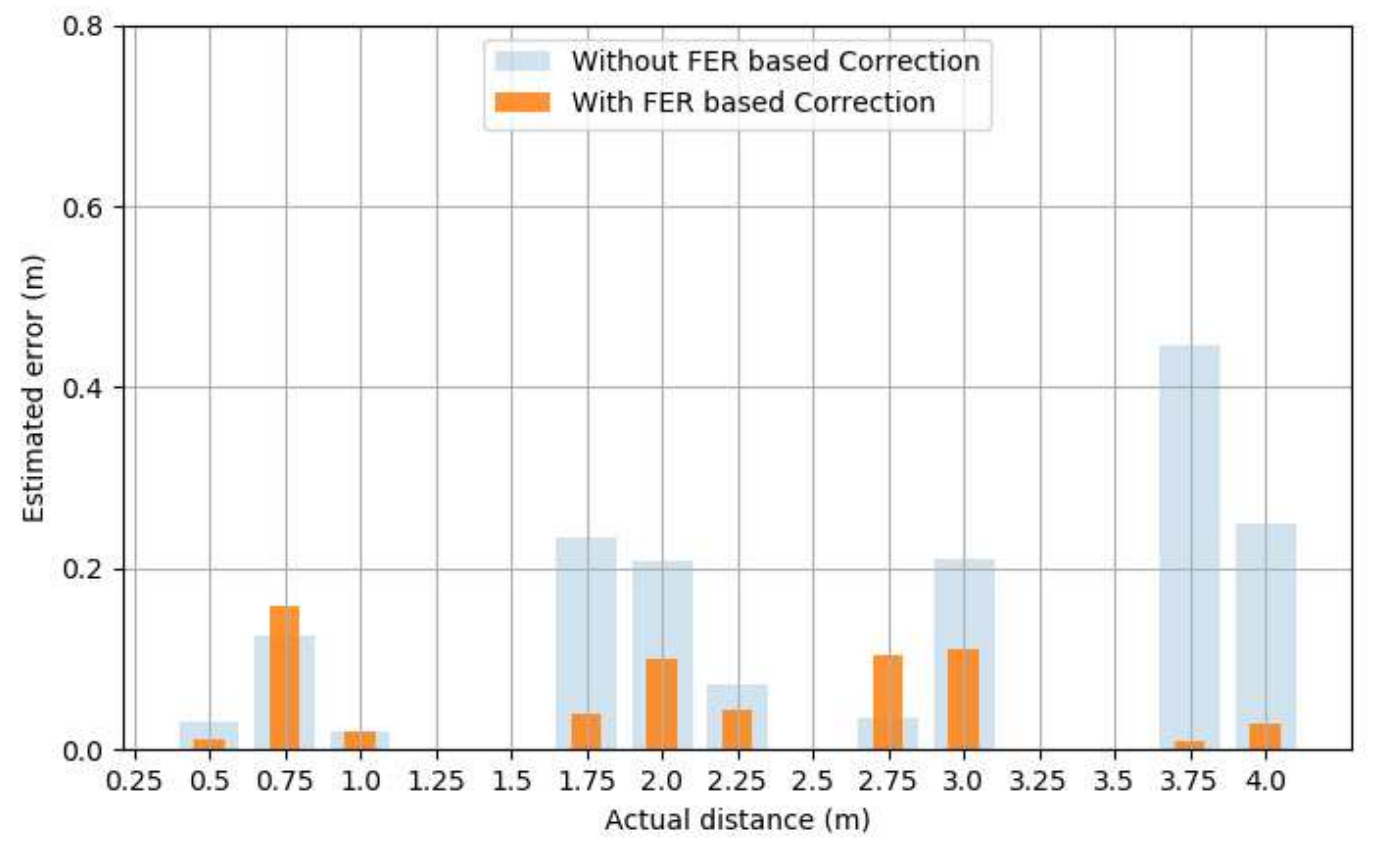

Figure 4.20: Actual distance and estimated error with and without $F E R$ based correction

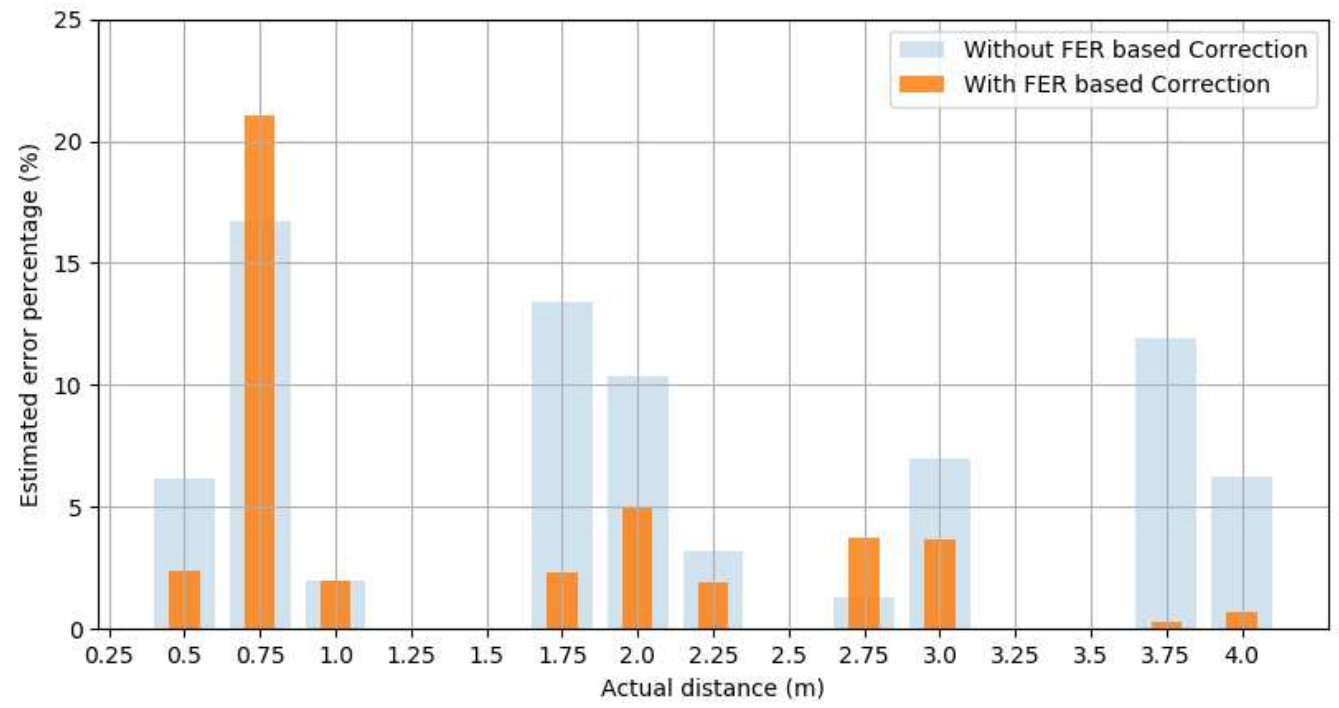

Figure 4.21: Actual distance and estimated error percentage with and without $F E R$ based correction

Graphs as shown in figure 4.20 and figure 4.21 represents the estimated error and estimated error percentage for with and without $F E R$ based correction respectively. 
Table 4.4: Error percentage in distance estimation with and without FER

\begin{tabular}{|l|l|l|l|}
\hline & Error Minimum (\%) & Error Maximum (\%) & Error Average (\%) \\
\hline With FER & 0.2825 & 21.0487 & 4.3013 \\
\hline Without FER & 2.0000 & 16.7395 & 7.8318 \\
\hline
\end{tabular}

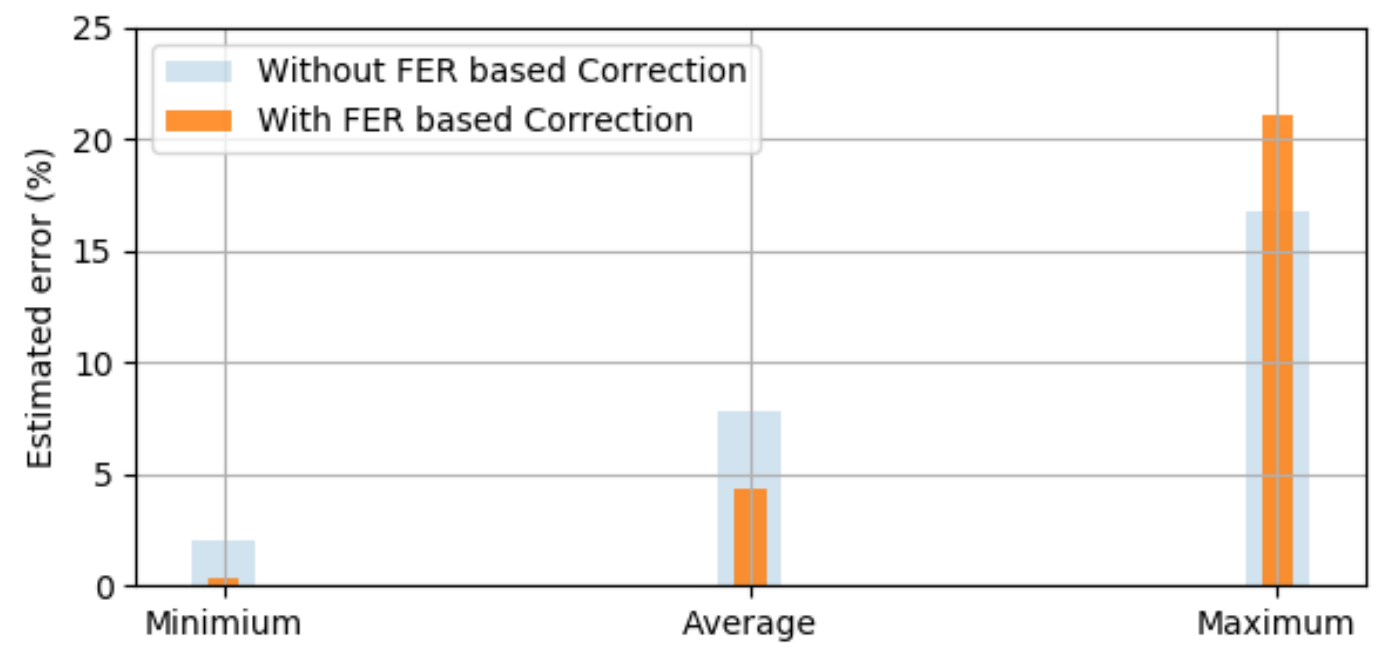

Figure 4.22: Error percentage in distance estimation with and without $F E R$

\subsubsection{Comparison with existing model}

Estimated error values for actual distance vs. estimated distance while considering with $F E R$ and without $F E R$ based correction of $R S S I$ for the graph scenario shown in figure 4.19 is represented in table 4.4 and in graph figure 4.22. In comparison with the existing model such as NLR (Zan Li et al., 2009) with WC-CWLS, average error of NLR model is $1.7 \mathrm{~m}$ and the average error of our model without FER based correction is $16.319 \mathrm{~cm}$ and with FER based correction is $6.242 \mathrm{~cm}$. Our model outperforms the existing models thereby concluding the consideration of FER for object localization implemented using IEEE $802.11 / \mathrm{a} / \mathrm{g} / \mathrm{p}$ based devices is needed.

Figure 4.23 shows various implementation stages during the experiment. Images shown in this figure represents the experimentation setup (1), high clutter region $(2,6,7)$, with obstacle (4), Line of Sight(LOS) (2) and Non-LOS diagonal (5) . Various implementation stages are discussed in the section 4.4.5. 

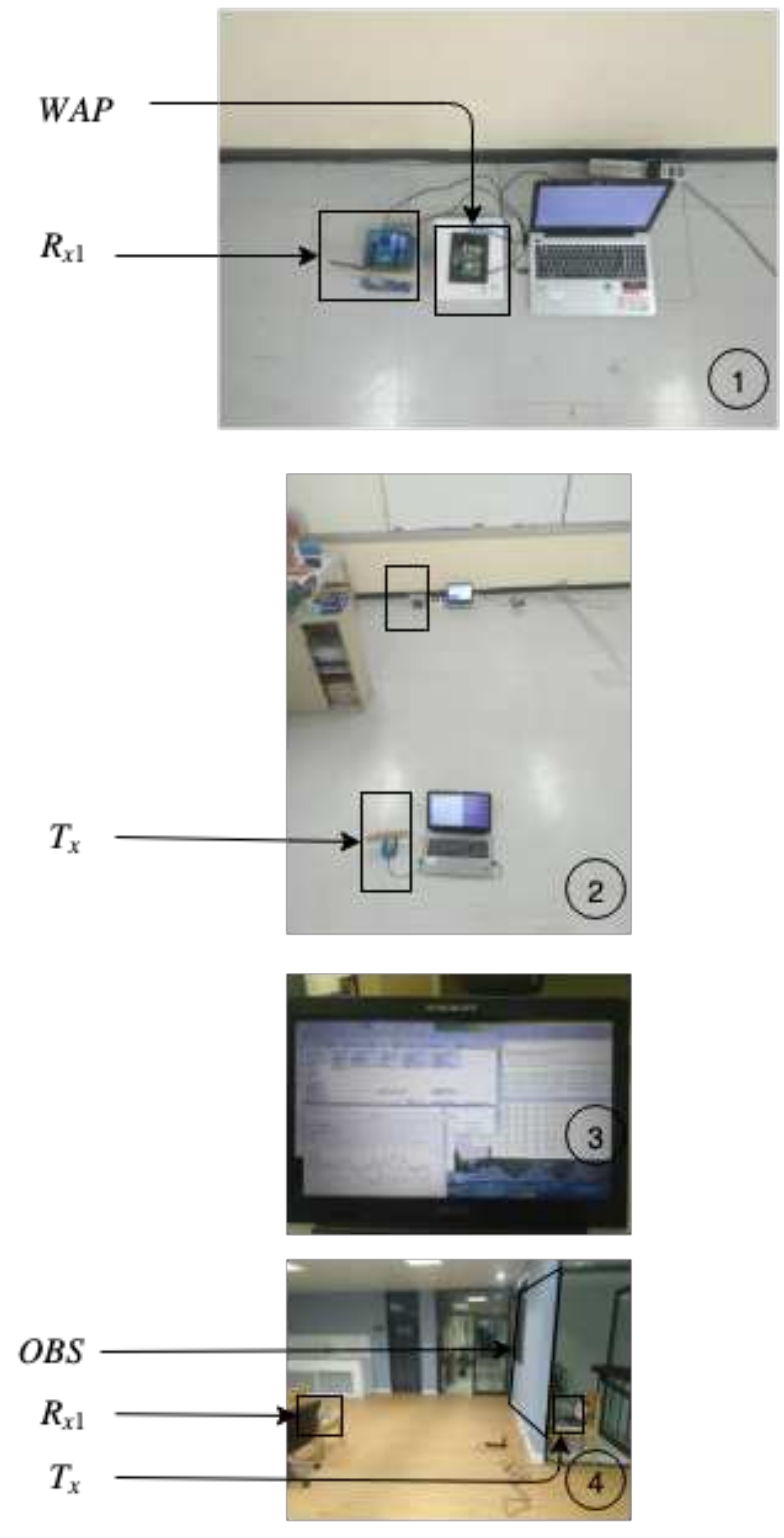

$R_{x 1}, R_{x 2}=$ Receiver $(s)$ $T_{x}=$ Transmitter WAP $=$ Wireless Access Point $O B S=$ Obstacle
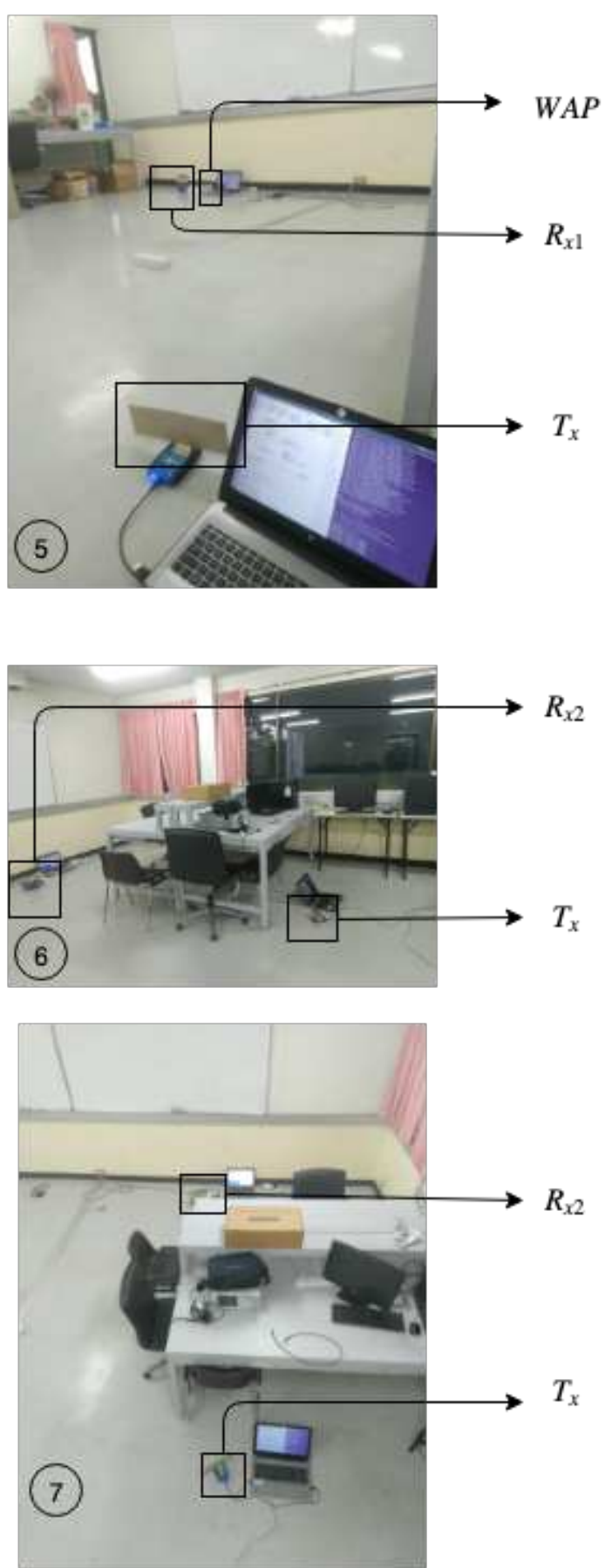

Figure 4.23: Experimentation setup at various stages 


\subsubsection{Implementation}

Different Implementation scenarios are discussed in figure 4.24, figure 4.27 and 4.29 with their high clutter environment in the Indoor. Their graphs with distance estimation is represented with calibrated RSSI based on respective FER. Graphs shown in figure 4.25 and figure 4.26 are for the implementation scenario represented in figure 4.24. Graphs shown in figure 4.28 is for the implementation scenario represented in figure 4.27. Graph shown in figure 4.30 and figure 4.31 are for the implementation scenario represented in figure 4.29

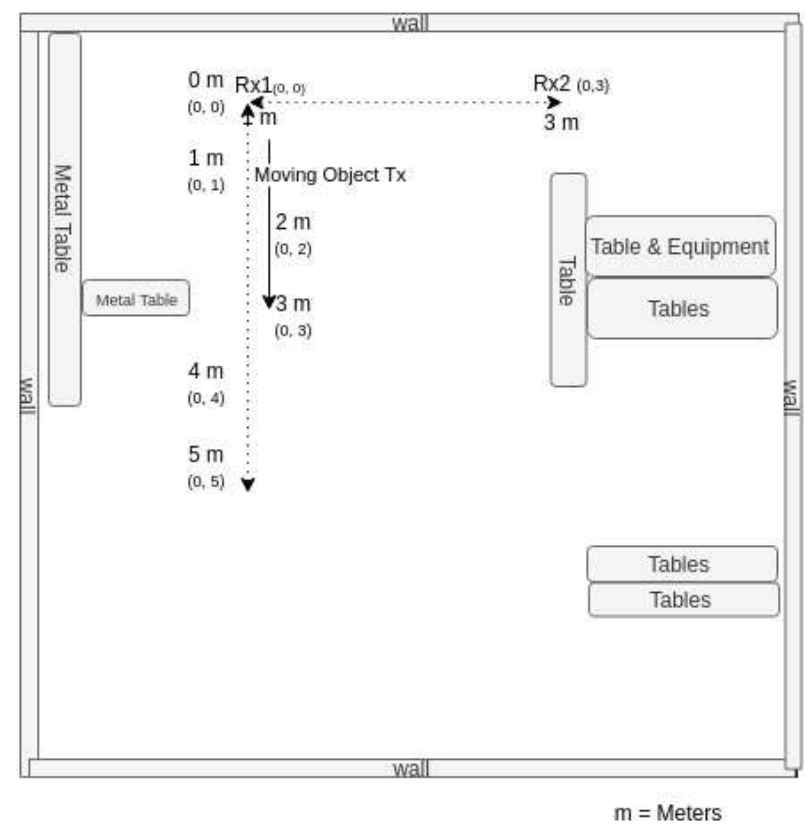

Figure 4.24: Tx moving away from $\mathrm{Rx} 1$ and $\mathrm{Rx} 2$ 


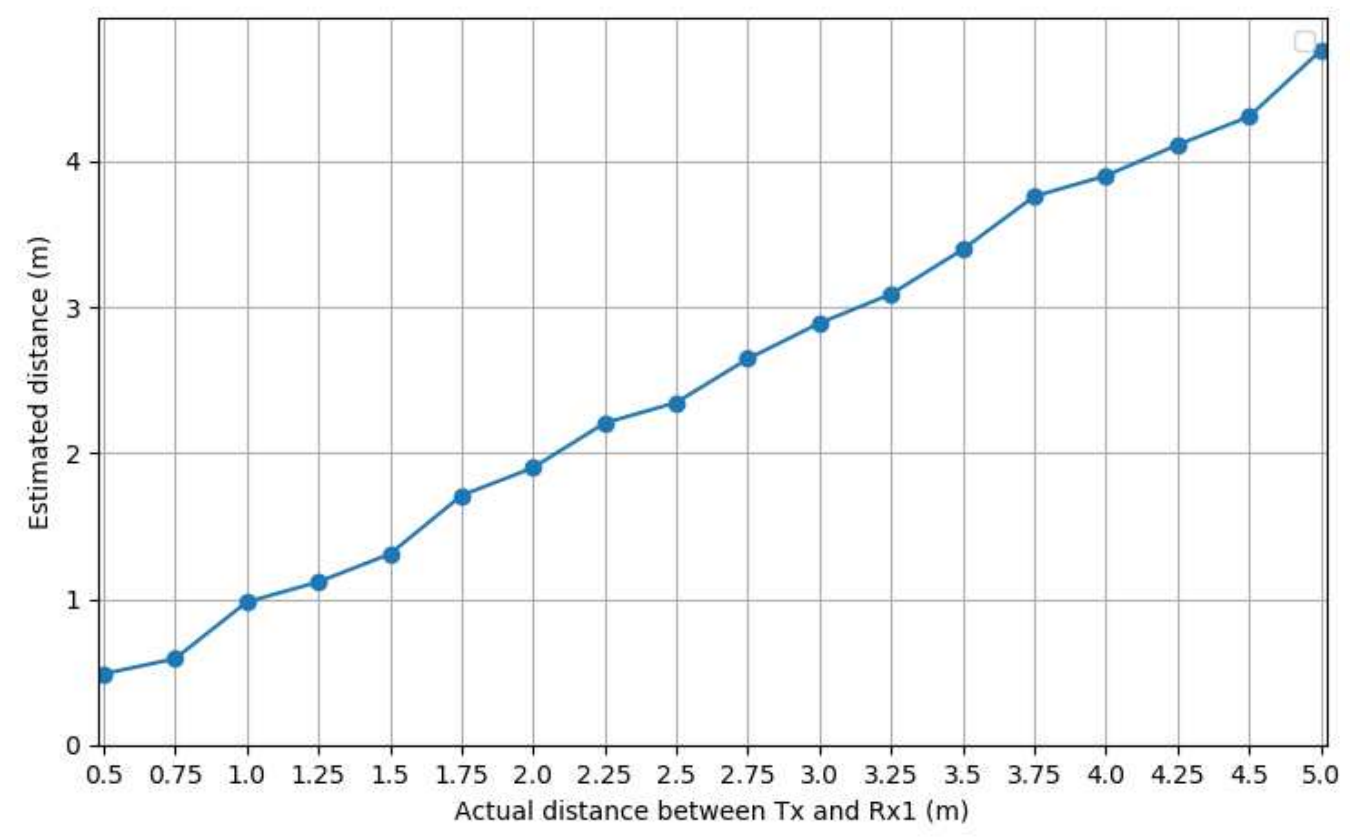

Figure 4.25: Distance estimated by Rx1 for Tx as shown in figure 4.24

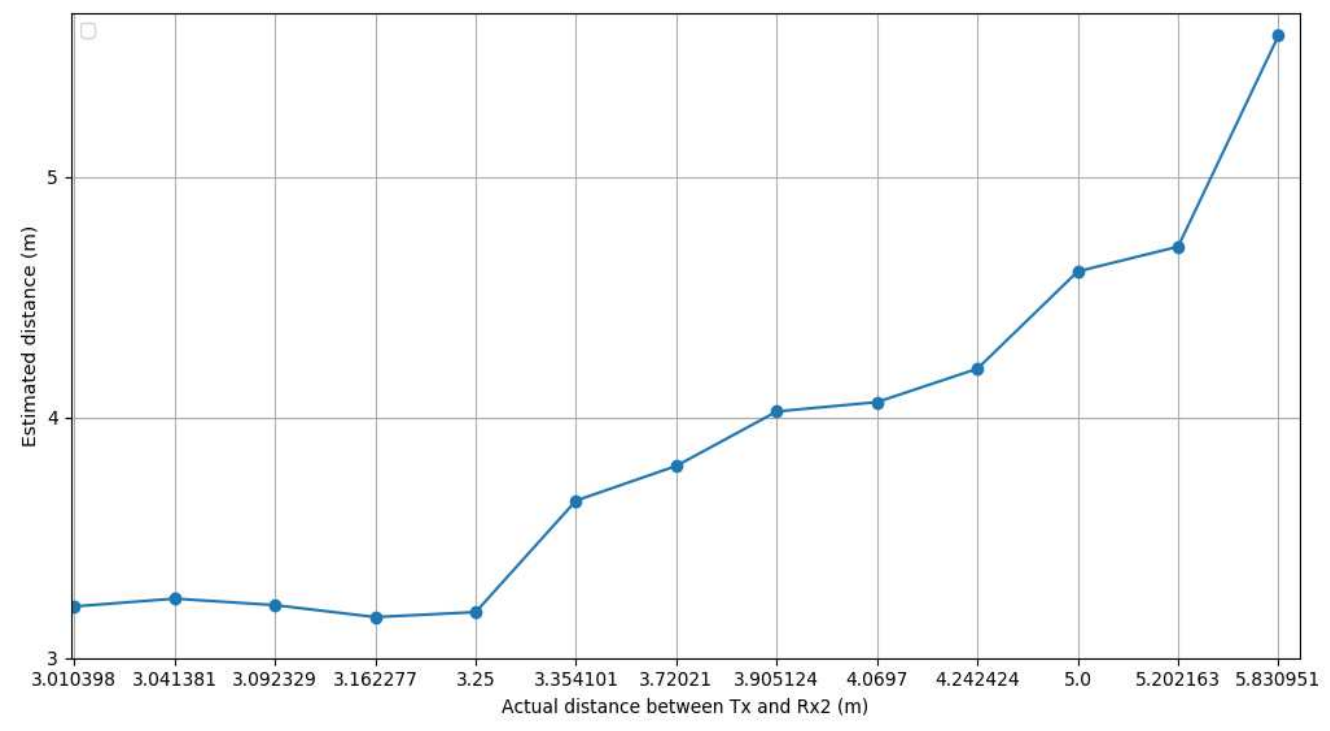

Figure 4.26: Distance estimated by $\mathrm{Rx} 2$ for $\mathrm{Tx}$ as shown in figure 4.24 


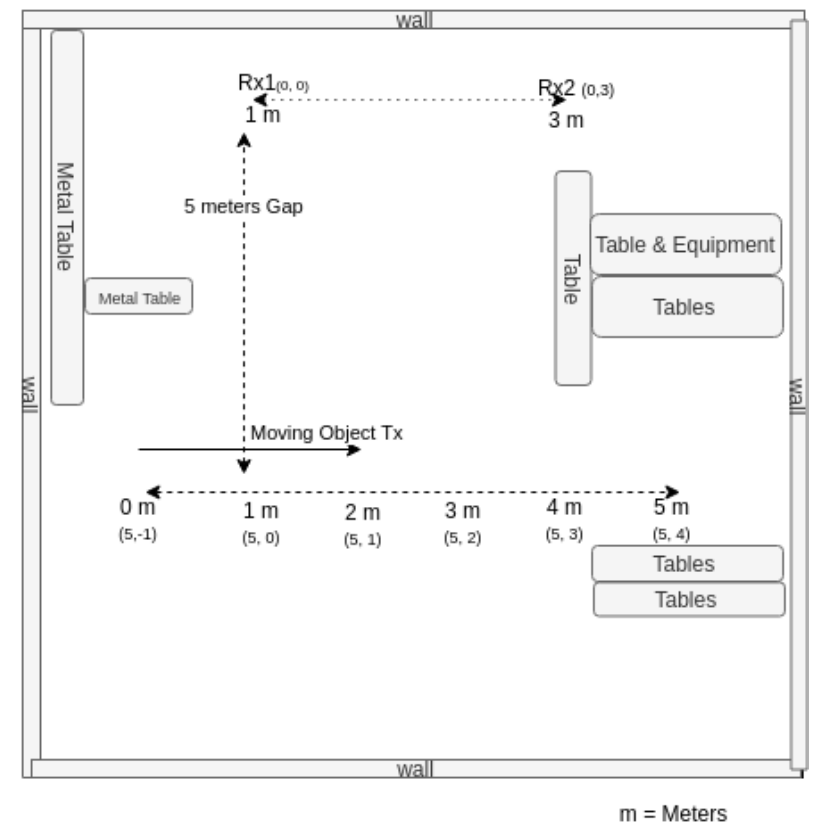

Figure 4.27: Tx is moved from Left to Right

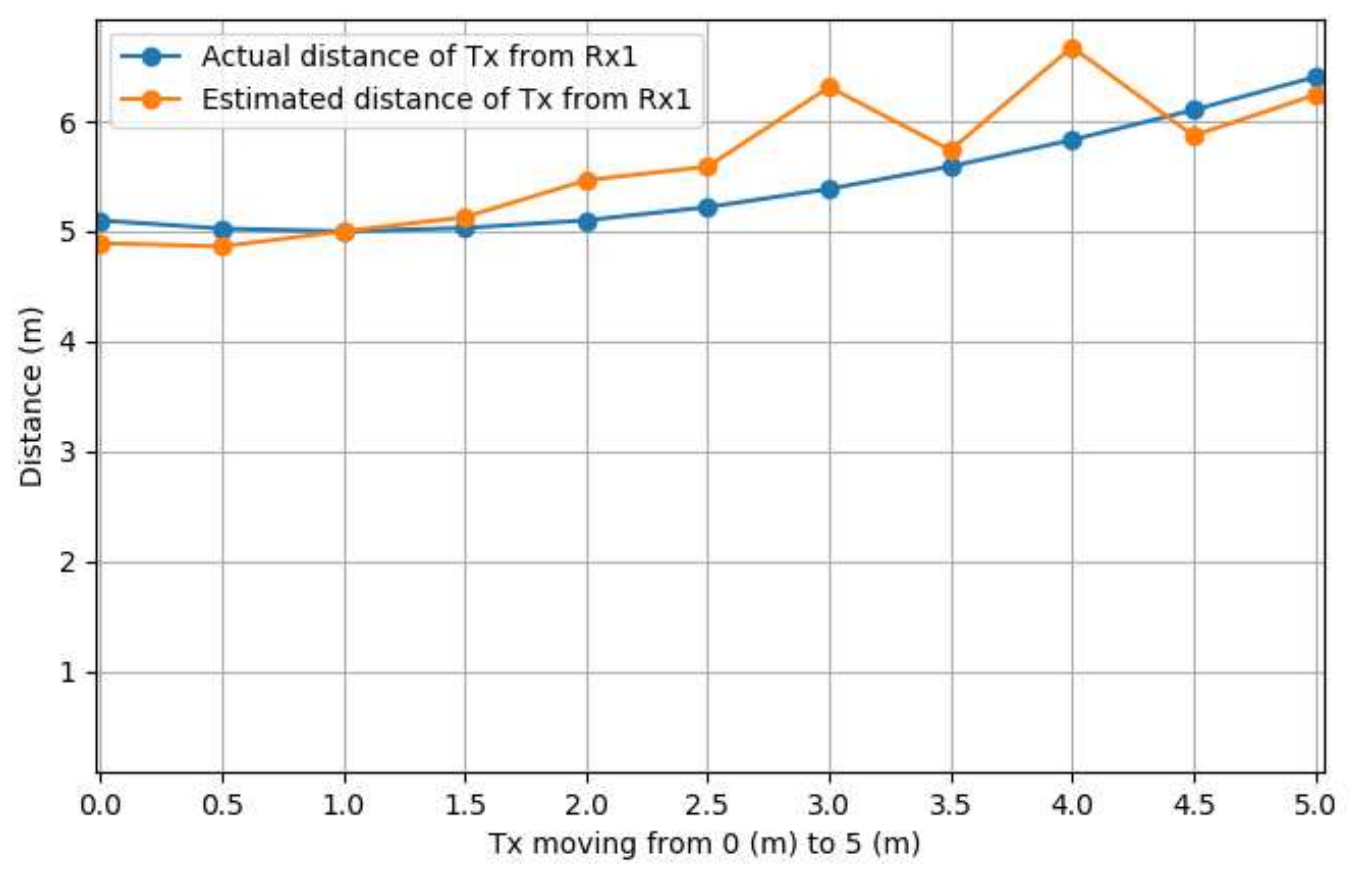

Figure 4.28: Distance estimated by $\mathrm{Rx} 1$ when Tx is moved as shown in figure 4.27 


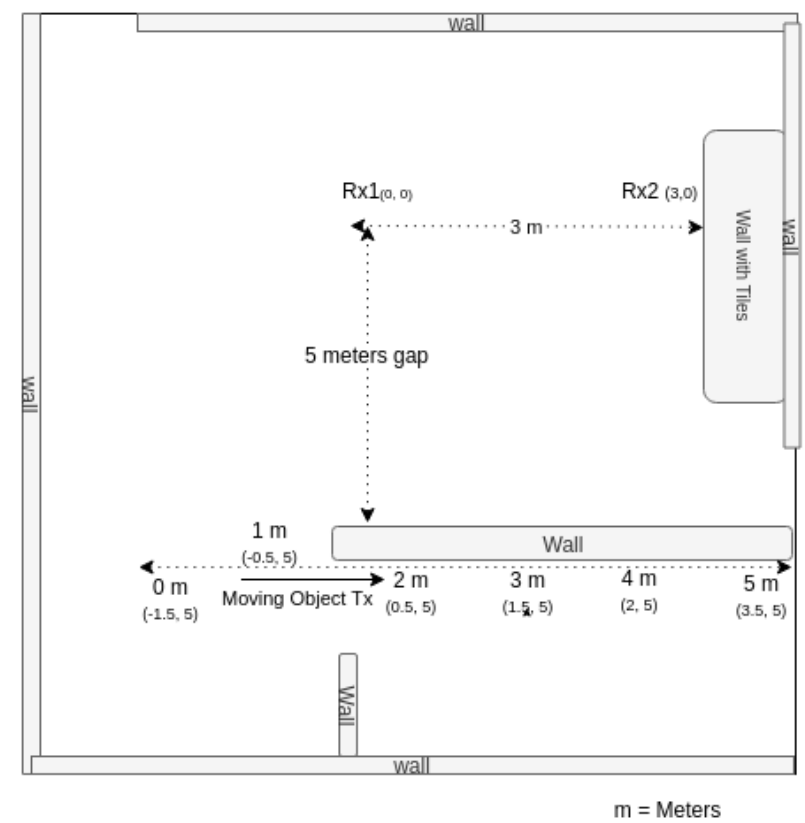

Figure 4.29: Obstacle between $\mathrm{Tx}$ and $\mathrm{Rx}$

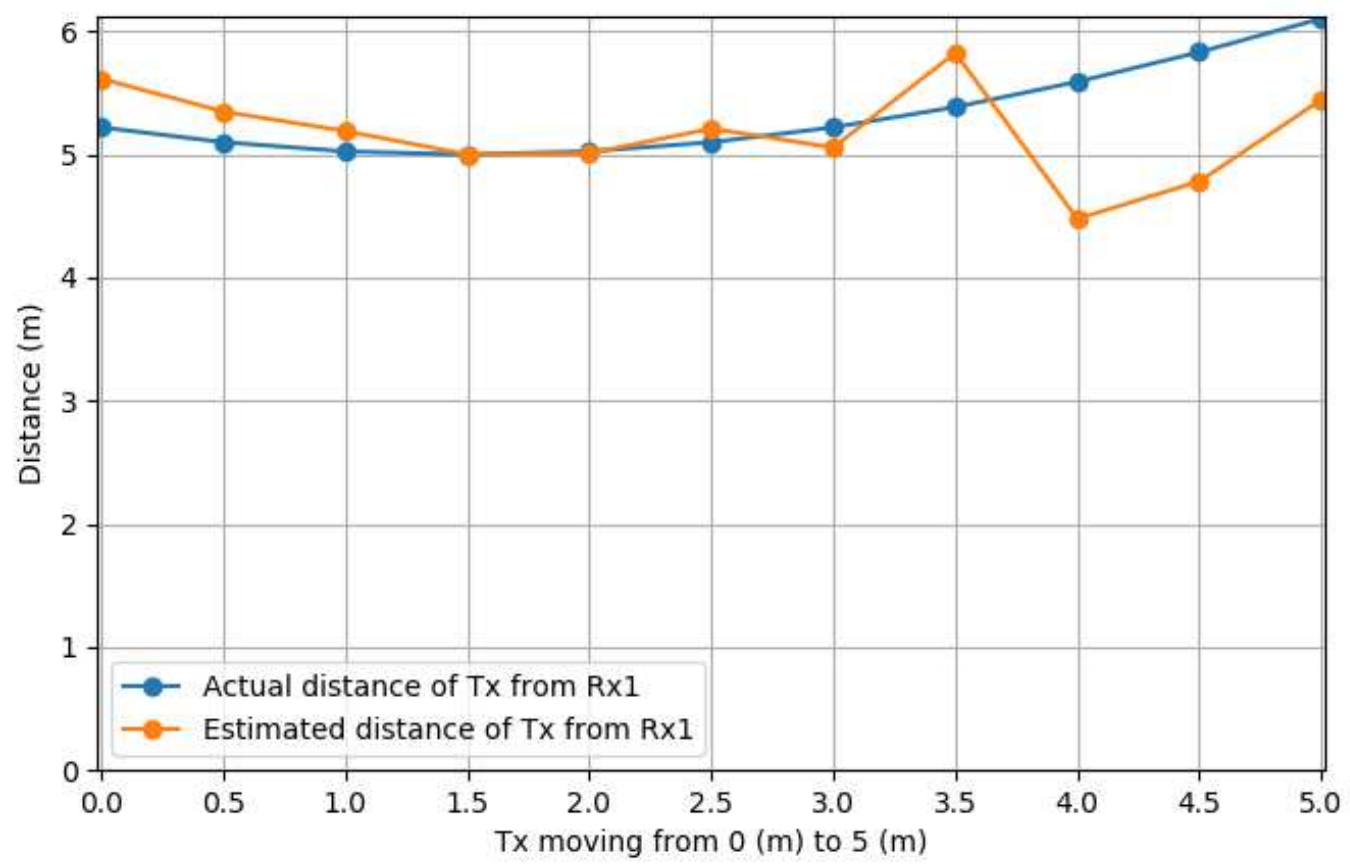

Figure 4.30: Distance estimated by Rx1 for Tx moving as shown in figure 4.29 


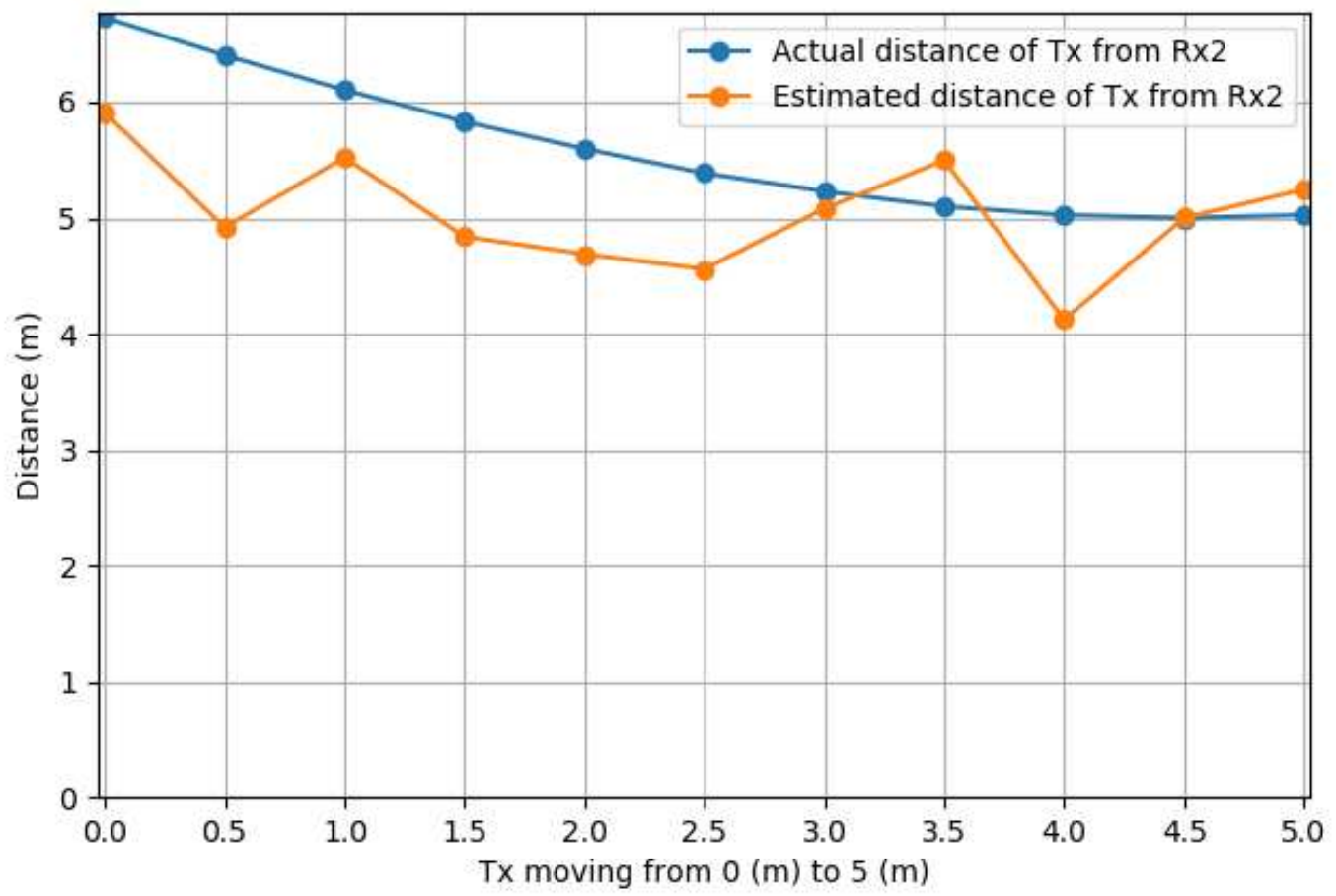

Figure 4.31: Distance estimated by $\mathrm{Rx} 2$ for Tx moving as shown in figure 4.29

On observation for the implementations as described in section 4.4.5, the mean error is less than $25 \mathrm{~cm}$, i.e for $5 \mathrm{~m}$, average observed distance is $4.75 \mathrm{~m}$. Mean error is $5 \%$ and mean Accuracy is $95 \%$. The maximum error is almost $90 \mathrm{~cm}$ for $5 \mathrm{~m}$ distance, i.e at $5 \mathrm{~cm}$, observed distance is $4.1 \mathrm{~m}$. Maximum error is $18 \%$ and minimum Accuracy is $82 \%$. 


\section{CHAPTER 5}

\section{CONCLUSION}

Conducted experiments showed that the use of the FER for correcting the RSSI for distance estimation provides an improvement in localization of objects. In this experiment, the methodological process for approximating the accurate value of RSSI based on FER led to achieving a minimum accuracy of $82 \%$ with a mean accuracy of $95 \%$ for object localization. During the process, an effort has been put on for analysing the samples of RSSI with respective to FER at various environments. The object of this work is to provide a novel framework for RSSI estimation considering FER with implementation on a low-cost system which can be used for object localization.

\section{Future Scope of the Research:}

In future, Single Board Computers (SBCs) can be used for object localization with inbuilt processing of the estimated algorithm. Further, this existing work can be used for improving the localization system by applying deep learning based techniques at the physical layer in wireless communications. Compressive Sensing (CS) can be used for intelligent estimation of spatial and temporal correlation of the Channel State Information (CSI) .

These systems should have capability to sense the compressed transmitted signal. These compressed signals are less prone to frame errors when compared to normal transmitted signal, besides this data driven deep learning algorithms employed at receiver can use predicted CSI based on Bayesian Estimation models for accurate object sensing and localization. 


\section{REFERENCE}

Adib, Mao, Kabelac, Katabi and Miller. (2015) Smart Homes that Monitor Breathing and Heart Rate. ACM.

Adib and Katabi. (2013). See Through Walls with Wi-Fi!. ACM.

Adib, Kabelac, Katabi and Miller. (2014) 3D Tracking via Body Radio Reflections. MIT.

Adib, Kabelac, Katabi and Miller. (2014) Multi-Person Localization via RF Body Reflections. MIT.

Ahmed, Talukder and Monjur. (2008). WiFi Radar: Design and Implementation of an Infrastructure-less Location Tracking System for Passive Environment. IEEE.

Andersen, Chee, M. Jacob, Pedersen, and Kurner. 2012. Reverberation and absorption in an aircraft cabin with the impact of passengers. IEEE Transactions.

Bamba, Joseph, B. Andersen, Tanghe, Vermeeren, Plets, Nielsen, and Martens. 2012. Experimental assessment of specific absorption rate using room electro magnetics. IEEE Transactions.

Chetty, Smith, Guo and Woodbridge. (2009). Target Detection in High Clutter using Passive Bistatic WiFi Radar. IEEE.

Chetty, Smith and Woodbridge. (2011). Through-the-wall Sensing of Personnel Using Passive Bistatic WiFi Radar at Standoff Distances. IEEE.

Falcone, Colone, Macera and Lombardo. (2012). Localisation and tracking of moving targets with WiFibased passive radar. IEEE.

Falcone, Colone, Bongioanni and Lombardo. (2010). Experimental Results for OFDM WiFi-Based Passive Bistatic Radar. IEEE.

Findlay and Dimbylow. 2008. Calculated SAR distributions in a human voxel phantom due to the reflection of electromagnetic fields from a ground plane between $65 \mathrm{MHz}$ and $2 \mathrm{GHz}$. Physics in Medicine and Biology.

Guo, Woodbridge, and C. J. Baker. (2008). Evaluation of Wifi Becon transmission for wireless based passive radar. IEEE.

GNU Radio with Raspberry pi for SDR applications,

https://www.rs-online.com/designspark/taking-the-raspberry-pi-2-

for-a-test-drive-with-gnu-radio-2

GNU radio for Raspberry pi,

https://github.com/scateu/GNURadioForRaspberryPiOnArchlinux

GNURadio, from

https://www.gnuradio.org/

Harima. 2012. Estimation of power absorbed by human body using reverberation cham- ber. IEEE. 
Hirata, Nagaya, Osamu, Nagaoka and Watanabe. 2007. Correlation between absorption cross section and body surface area of human for far-field exposure at GHz bands. IEEE. International Symposium on Electromagnetic Compatibility.

Hostpd for Raspberry pi,

https://wireless.wiki.kernel.org/en/users/documentation/hostapd

Hurst and Ellingson. 2008. Path loss from a transmitter inside an aircraft cabin to an exterior fuselagemounted antenna.. IEEE Transactions.

Kientega, Conil, Hadjem, Richalot, Gati, Wong, Picon, and Wiart. 2011. A surrogate model to assess the whole body sar induced by multiple plane waves at $2.4 \mathrm{GHz}$. annals of telecommunications - annales des tlcommunications.

Melia, G. (2013). Electromagnetic Absorption by the Human Body from 1 to $15 \mathrm{GHz}$. (Doctoral thesis, The University of York).

Mishra and Potnis. (2017). Wireless Transmission Detection and Monitoring System using GNU Radio and Multiple RTL-SDr Receivers. International Journal of Engineering Research and Application.

Nezirovic A., Liu M., Yarovoy A.G. 2007. Modelling of Reflection of UWB Pulses from Trapped Human Beings. In: Baum C.E., Stone A.P., Tyo J.S. (eds) Ultra-Wideband Short-Pulse Electromagnetics 8. Springer, New York, NY.

Pluto sdr,

https://wiki.analog.com/university/tools/pluto

Robinson, Clegg, and Marvin. Radio frequency electromagnetic fields in large conducting enclosures: effects of apertures and human bodies on propagation and field- statistics. IEEE Transactions on Electromagnetic Compatibility.

Rzewuski and Kulpa. (2011). System Concept of WIFI Based Passive radar. INTL JOURNAL OF ELECTRONICS AND TELECOMMUNICATIONS.

Raspberry pi 3,

https://www.raspberrypi.org/products/raspberry-pi-3-model-b/ 


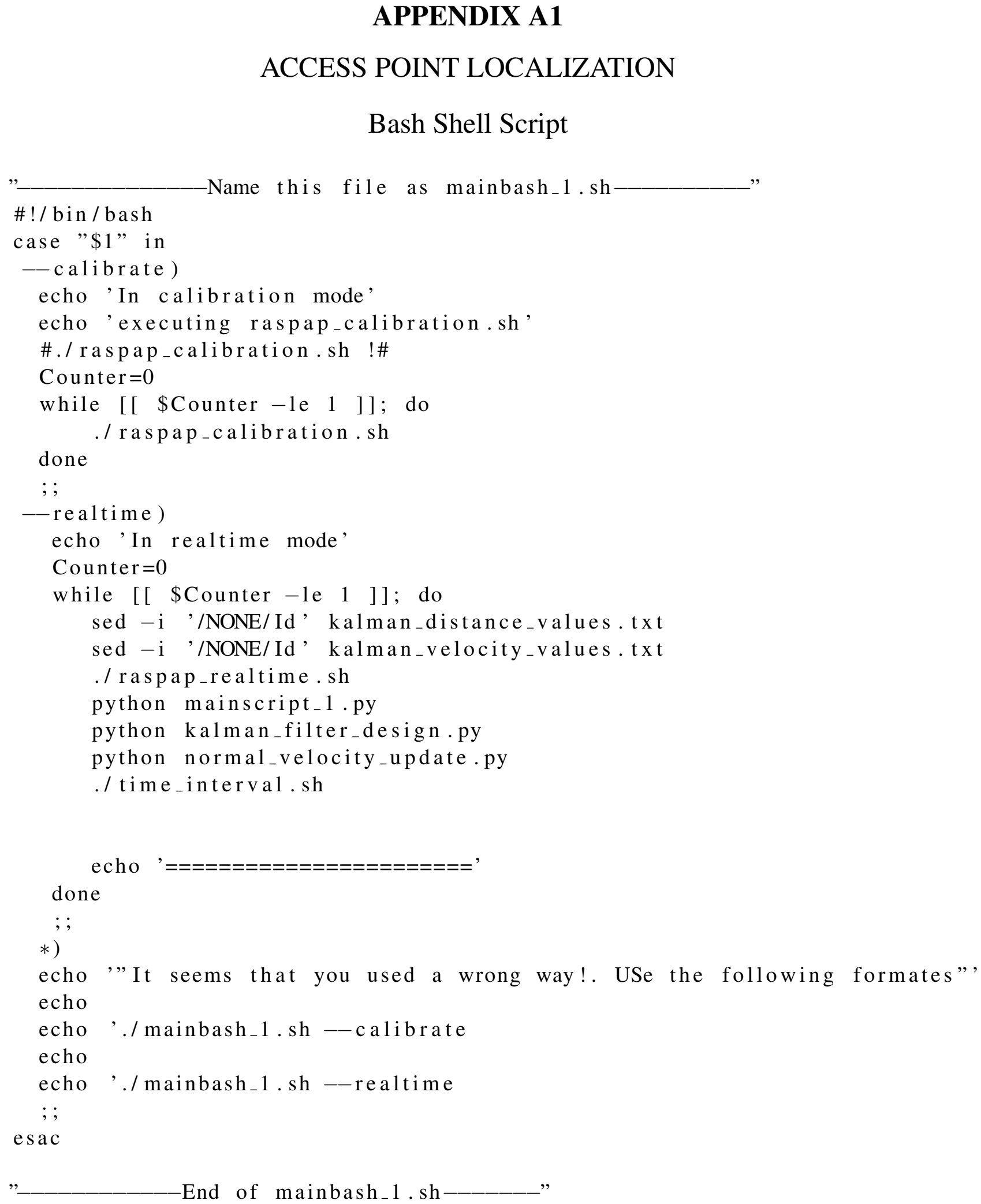




\section{APPENDIX A2}

\section{ACCESS POINT LOCALIZATION}

\section{Bash Shell Script}

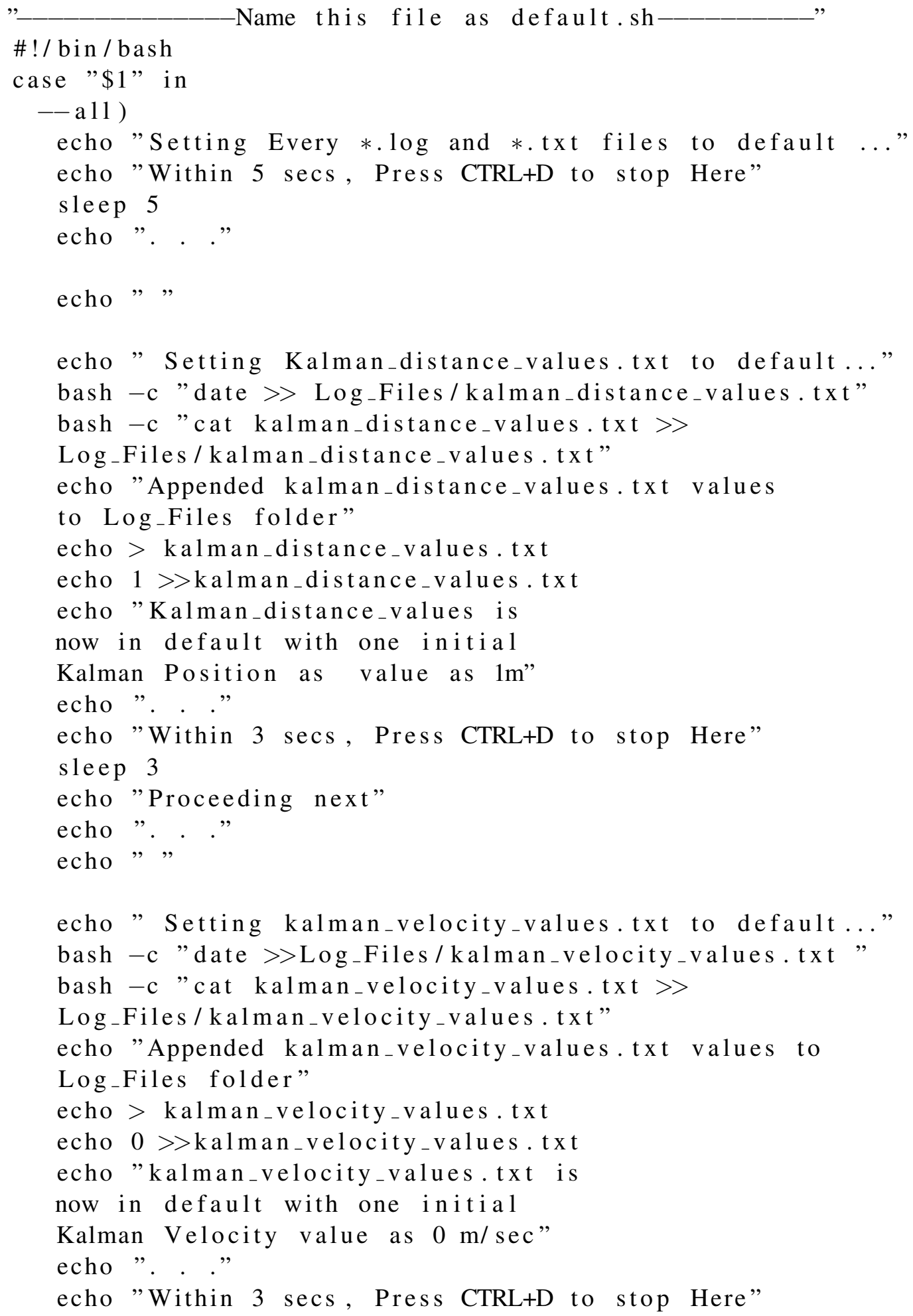




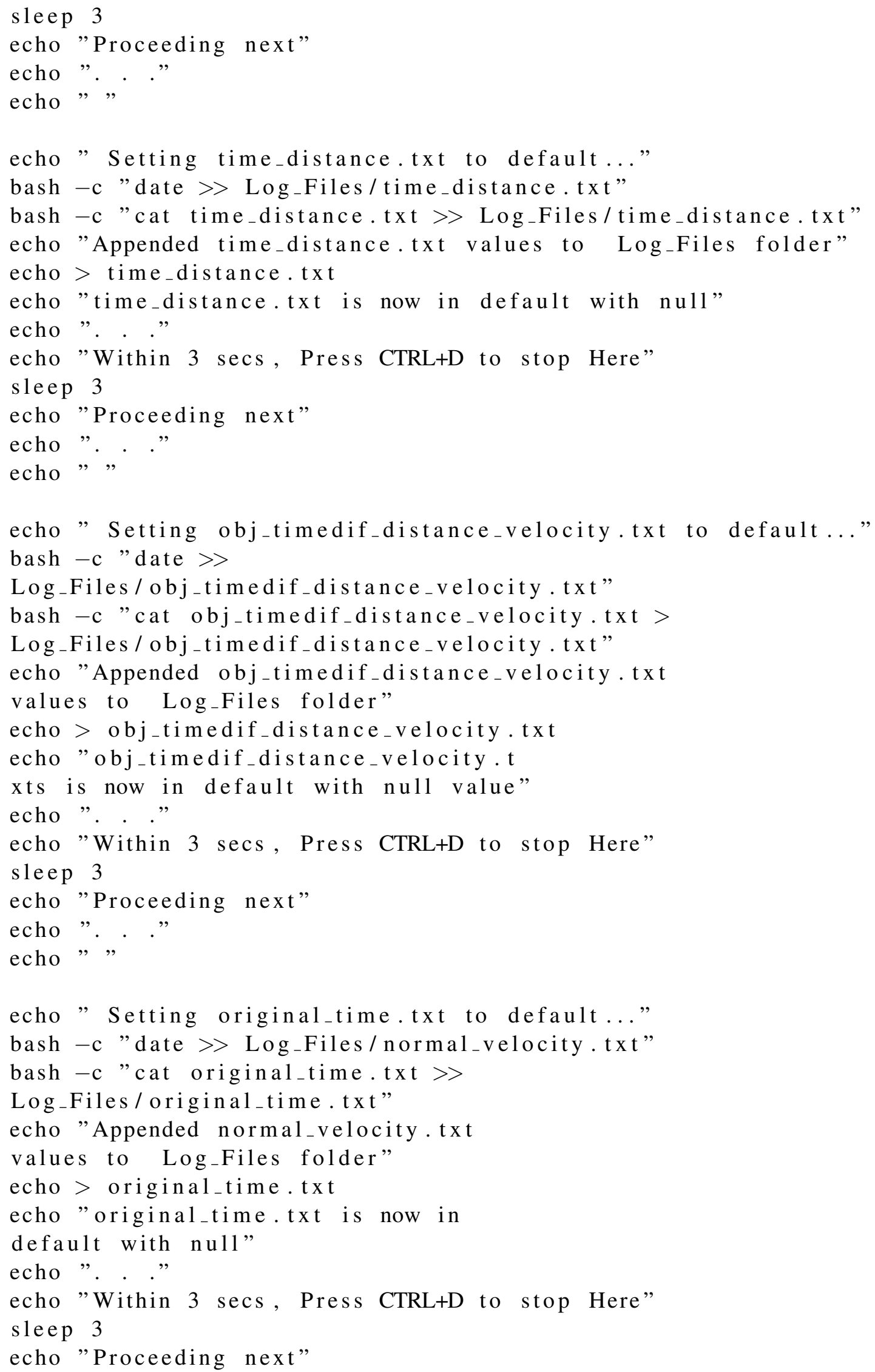




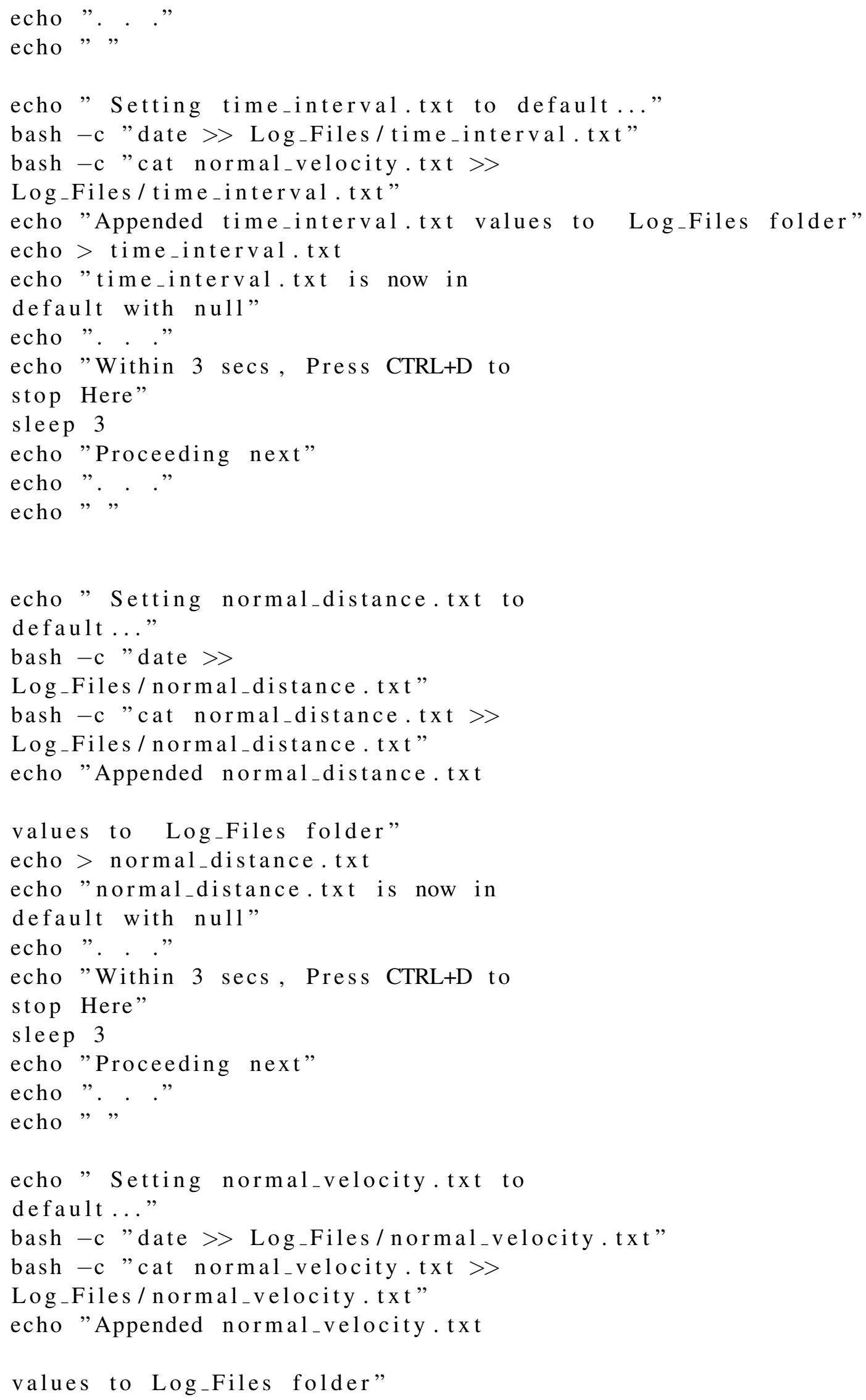




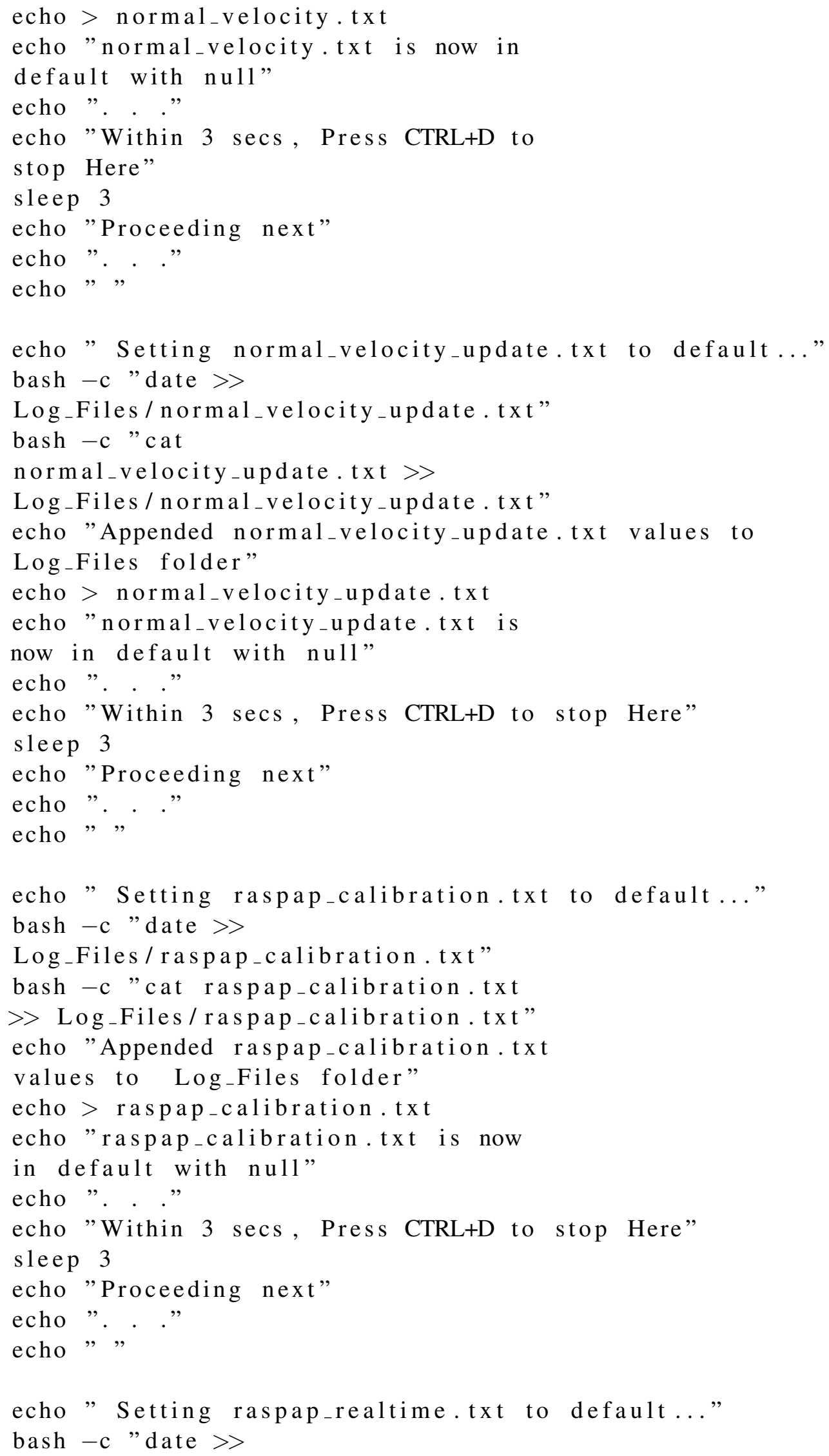


Log_Files/raspap_realtime.txt"

bash $-c$ "cat raspap_realtime.txt $>$

Log_Files / raspap_realtime.txt"

echo "Appended raspap_realtime.txt

values to Log_Files folder"

echo $>$ raspap_realtime.txt

echo "raspap_realtime.txt is now in

default with null"

echo "... ."

echo "Within 3 secs, Press CTRL+D to

stop Here"

sleep 3

echo "Proceeding next"

echo ". . ."

echo" "

echo ". . . . . . . . . . . . . . . . . . ."

echo "Restored these to defaults:

kalman_distance_values.txt, kalman_velocity.txt,

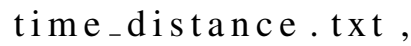

$o_{\text {obj }}$ timedif_distance_velocity.txt,

normal_distance.txt, normal_velocity.txt,

raspap_calibration.txt and raspap_realtime.txt"

; ;

- help)

echo "run 'default.sh' for making

kalman_distance_values.txt,

kalman_velocity.txt,

time_distance.txt,

obj_timedif_distance_velocity.txt,

normal_distance.txt,

normal_velocity.txt, to defalt

initial state leaving

'raspap_calibration.txt' \&

'raspap_realtime.txt, in the current state"

echo "run' default.sh - all' for making every *.log and *.txt

files to its default initail state..."

echo "run 'default.sh - -help' for any

help"

$; ;$

$*)$

echo "Setting *. log and *.txt files to default state except

'raspap_calibration.txt' \&

'raspap_realtime.txt, ..."

echo "Within 5 secs, Press CTRL+D to stop Here"

sleep 5

echo ". . ." 


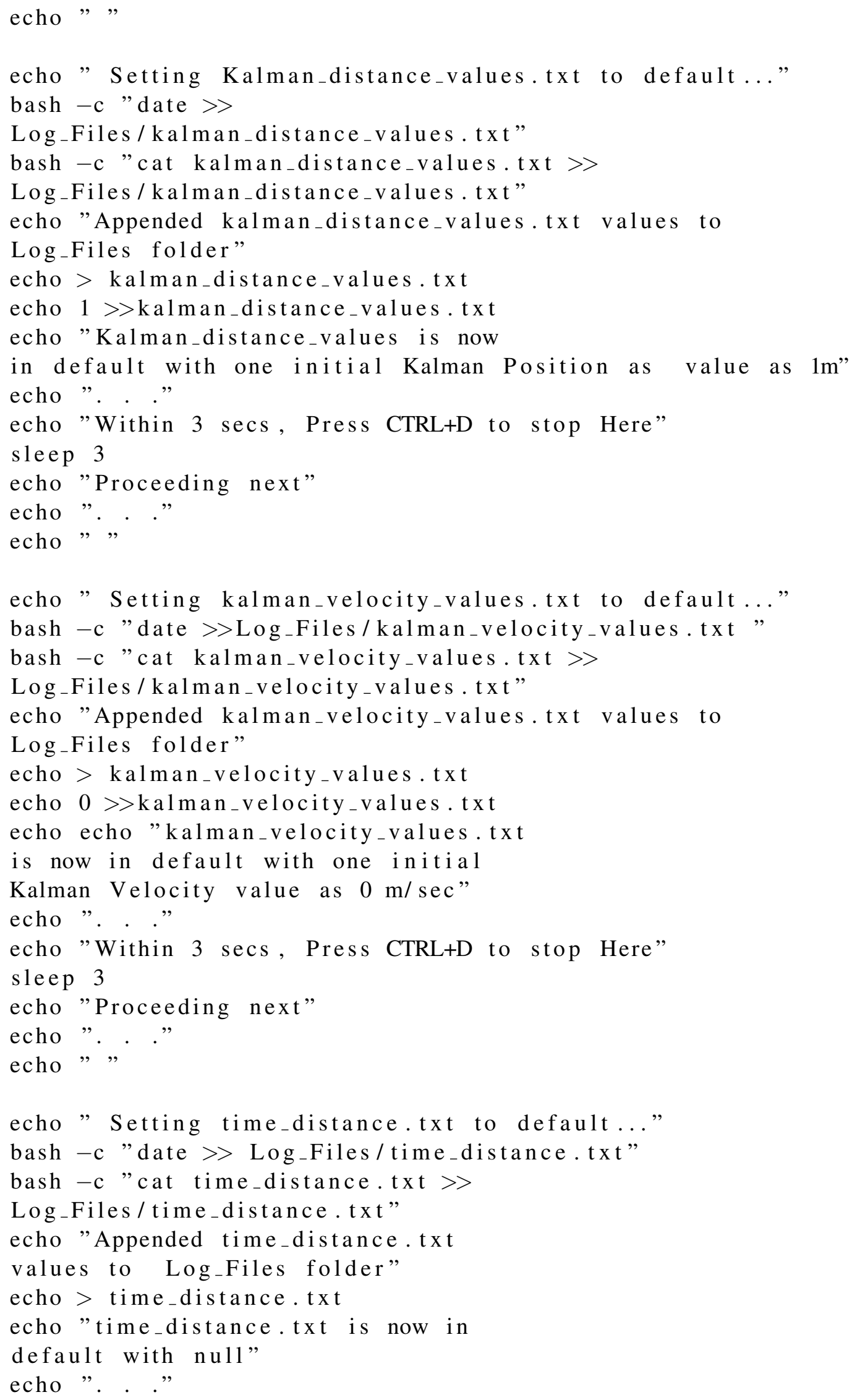




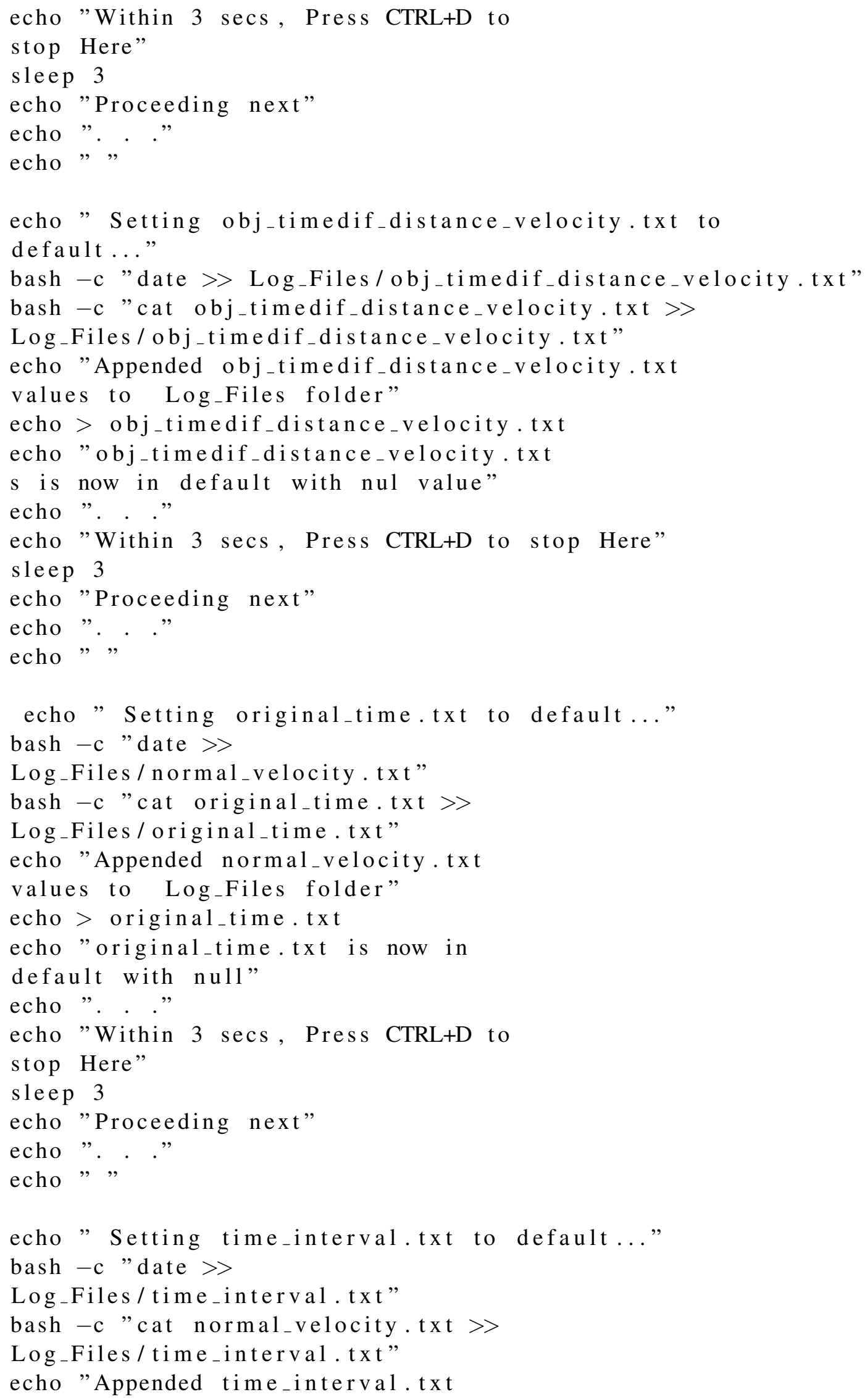




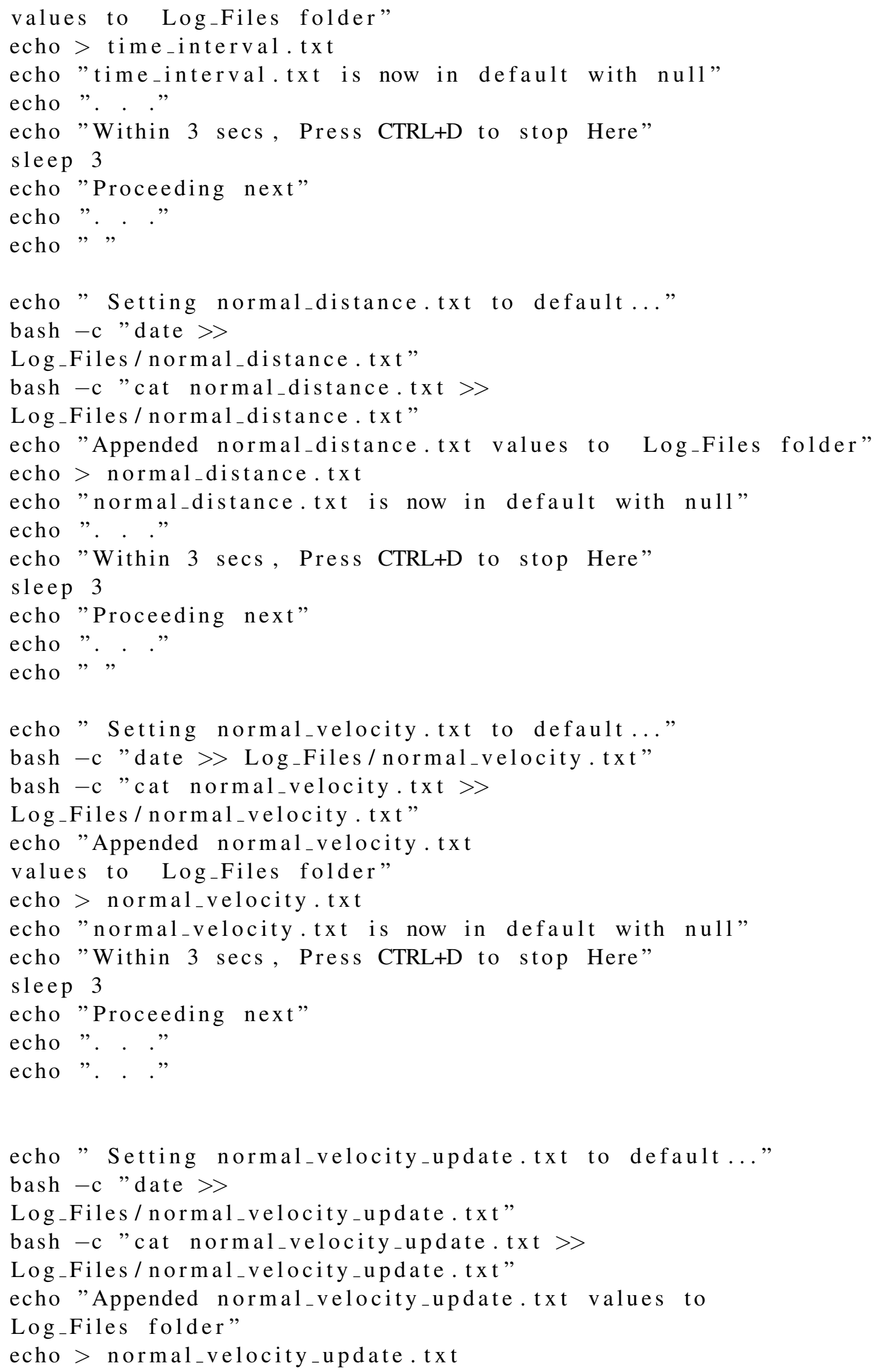




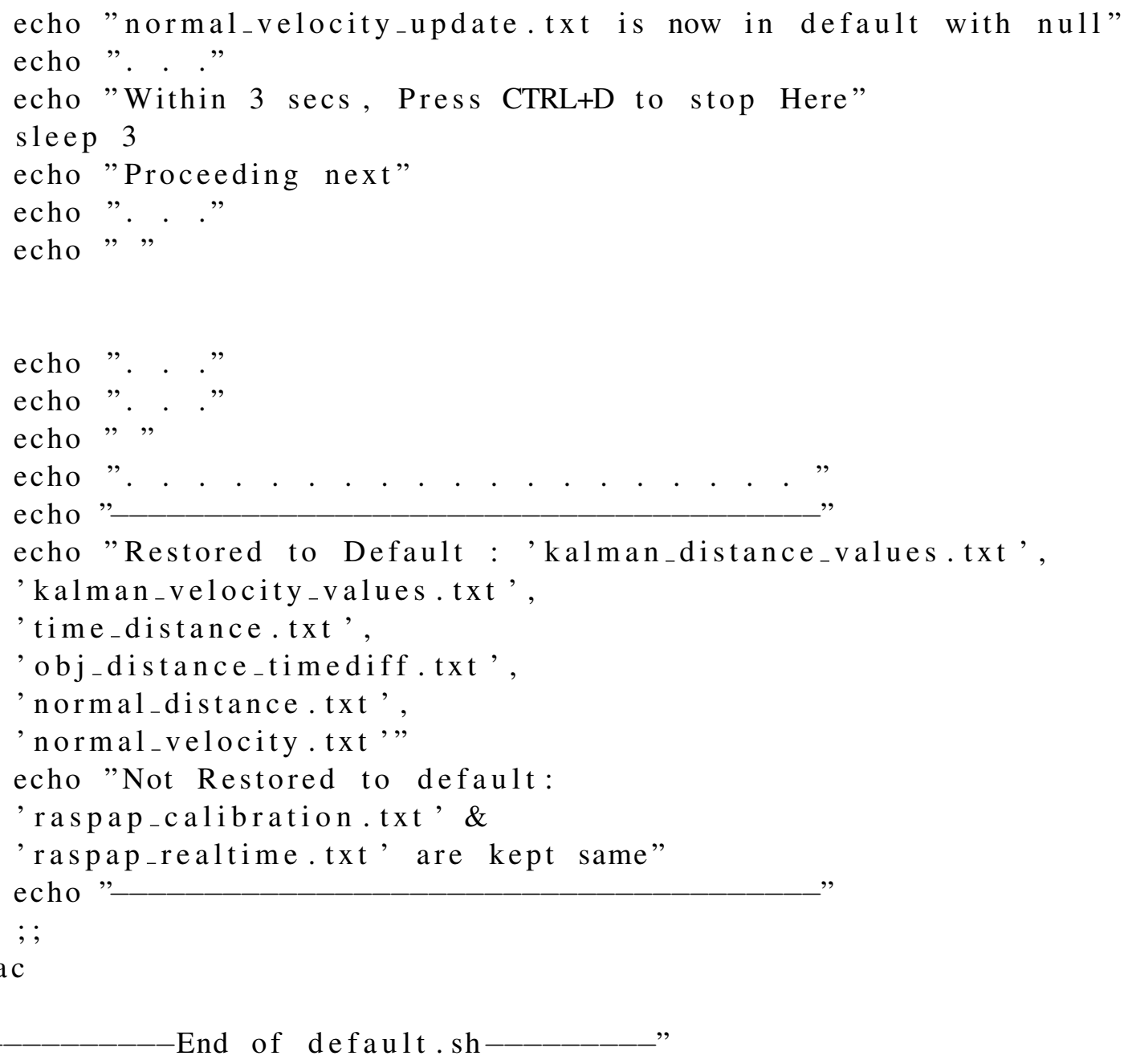




\section{APPENDIX A3 \\ ACCESS POINT LOCALIZATION}

\section{Bash Shell Script}

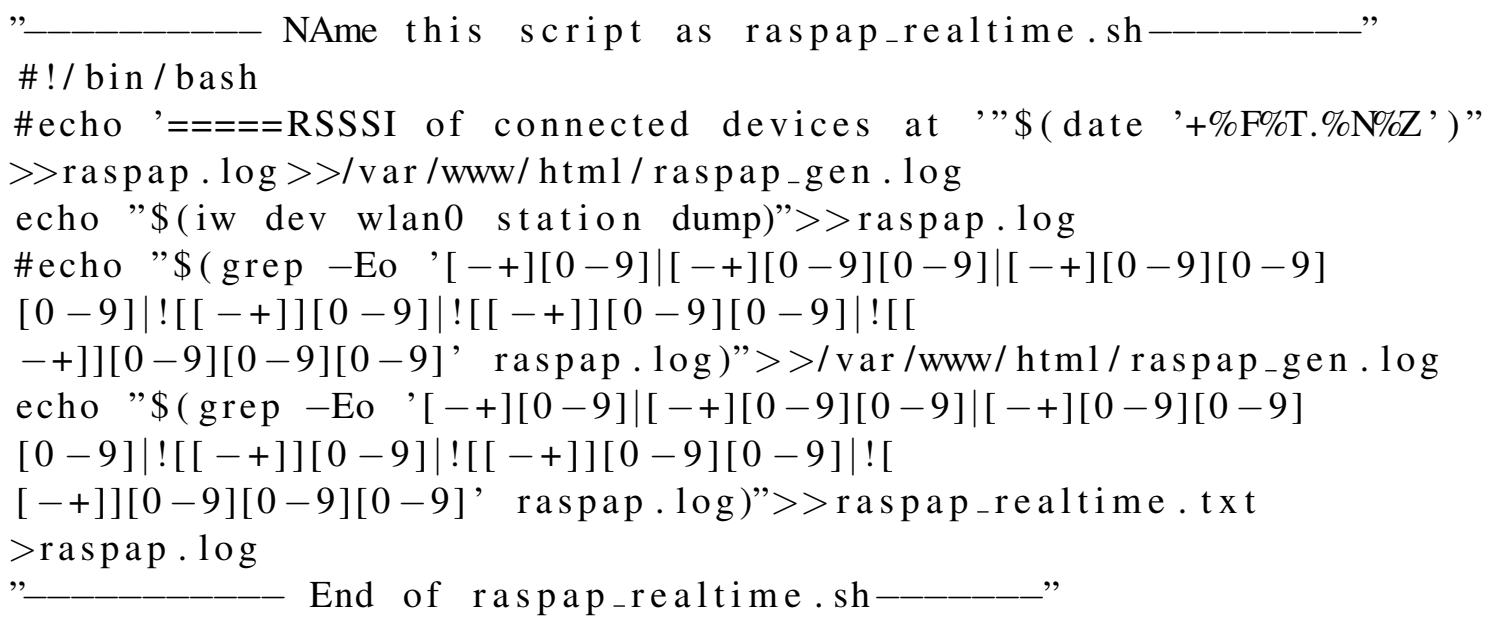

\section{APPENDIX A4}

ACCESS POINT LOCALIZATION

\section{Bash Shell Script}

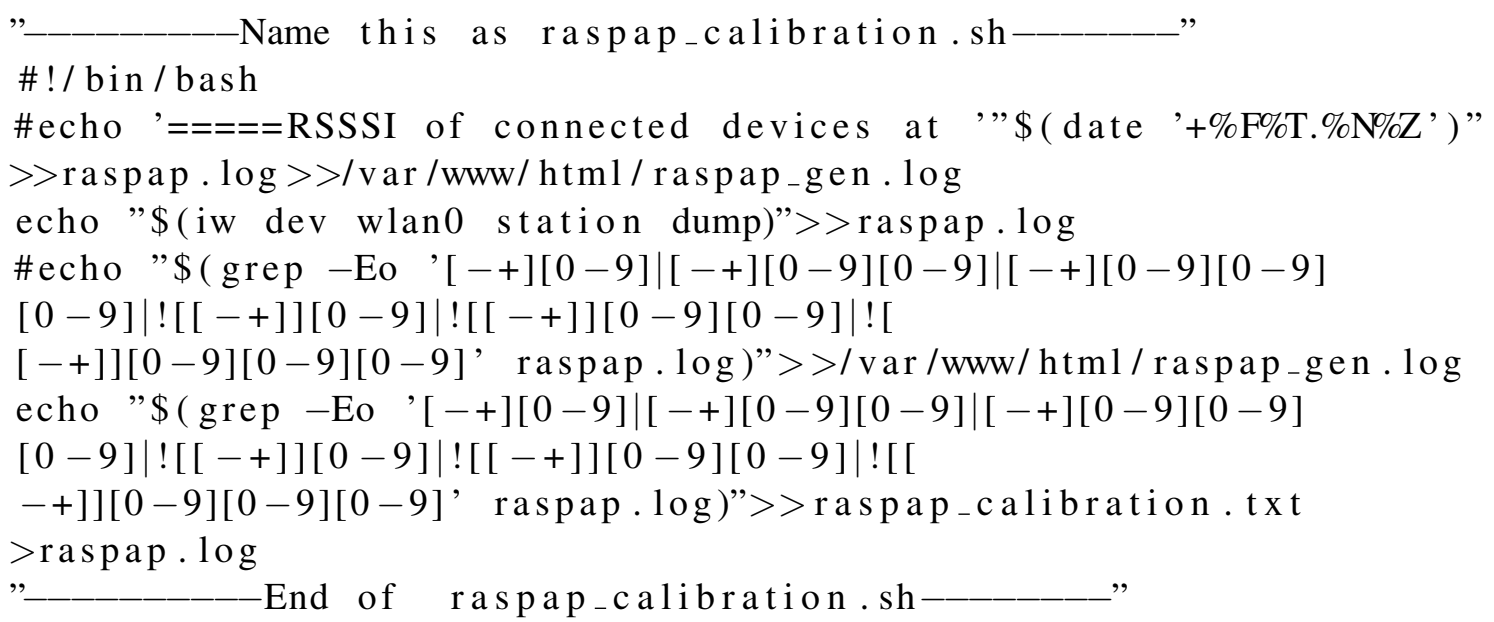




\section{APPENDIX A5 \\ ACCESS POINT LOCALIZATION \\ Python Code}

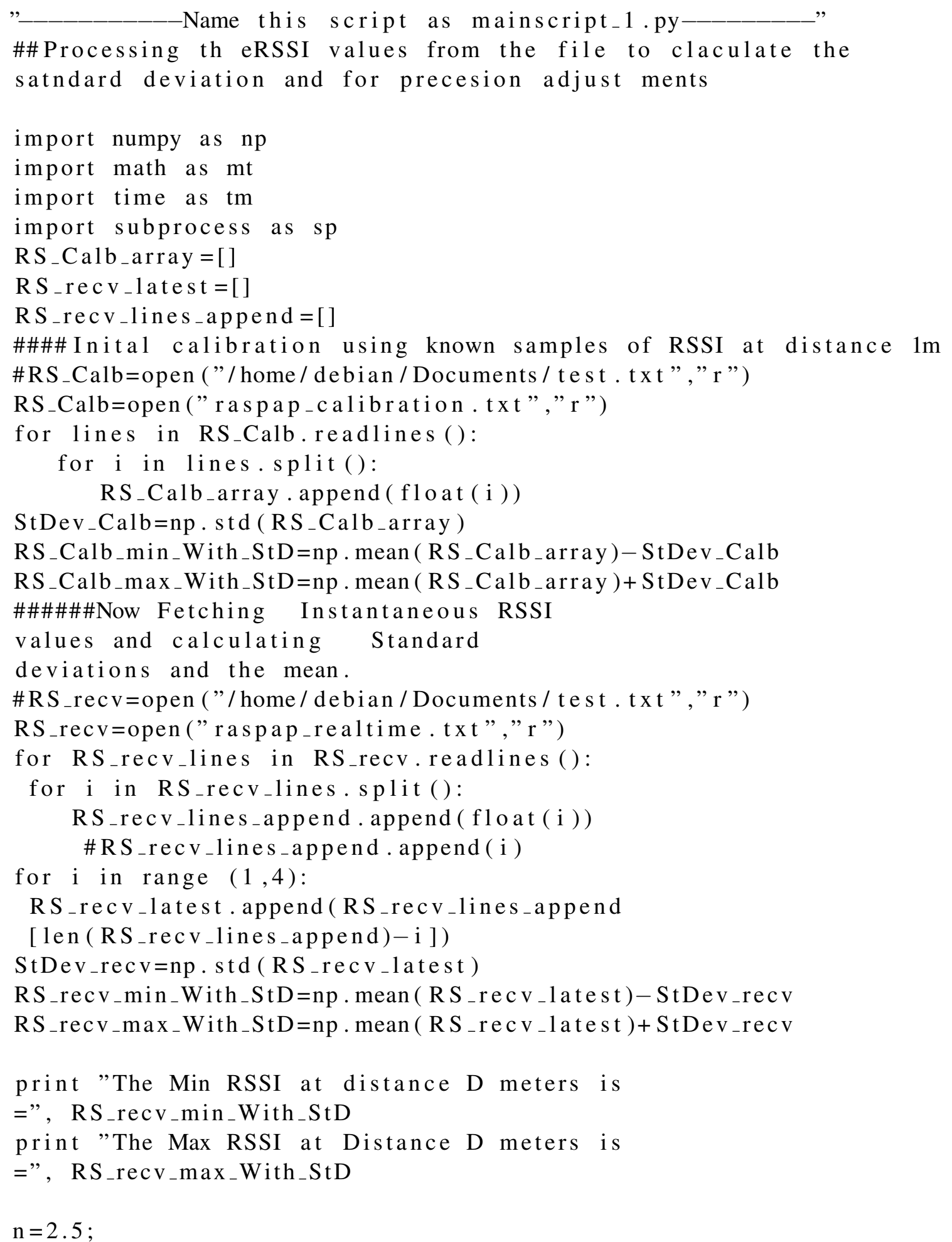




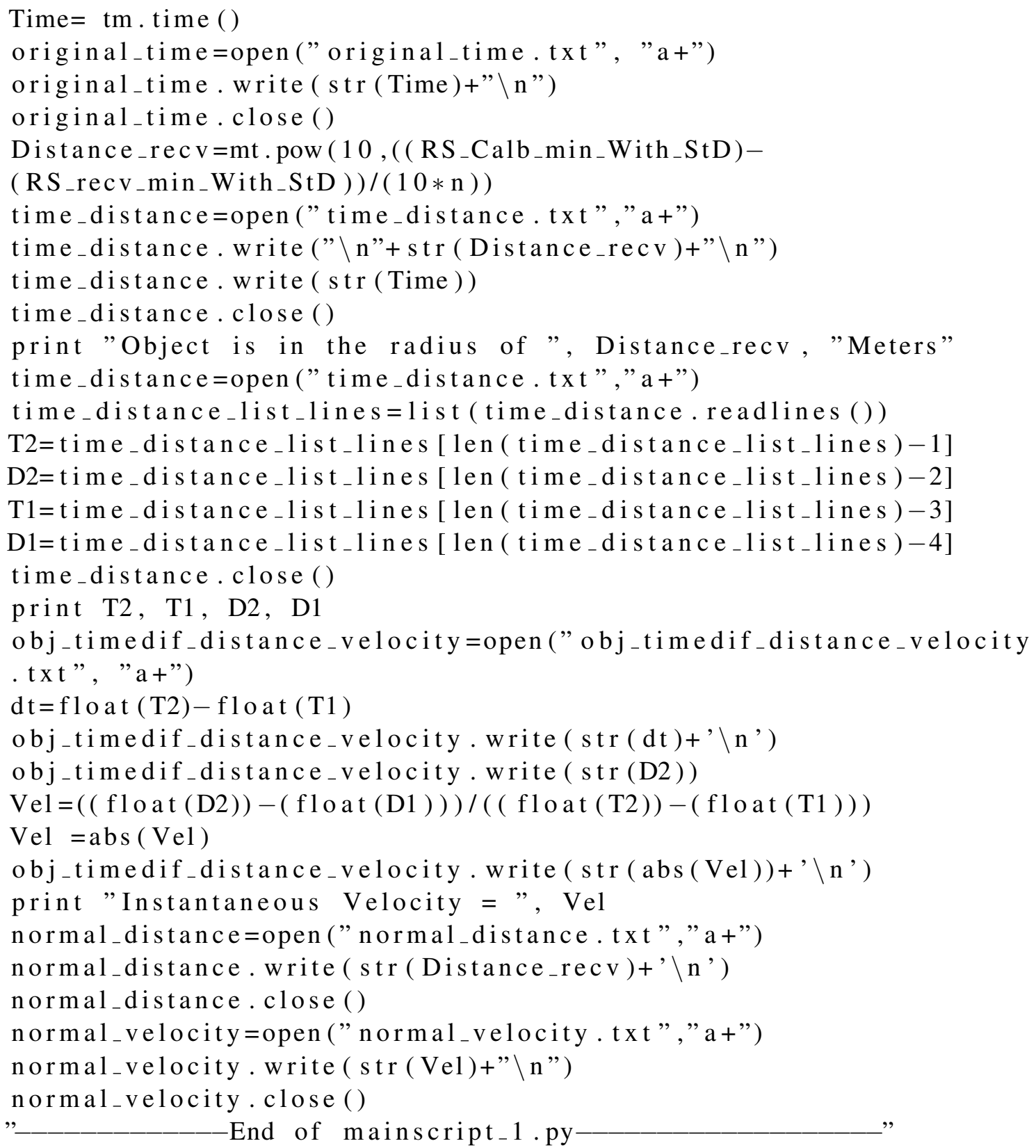




\section{APPENDIX A6 \\ ACCESS POINT LOCALIZATION \\ Kalman Filter Python Code}

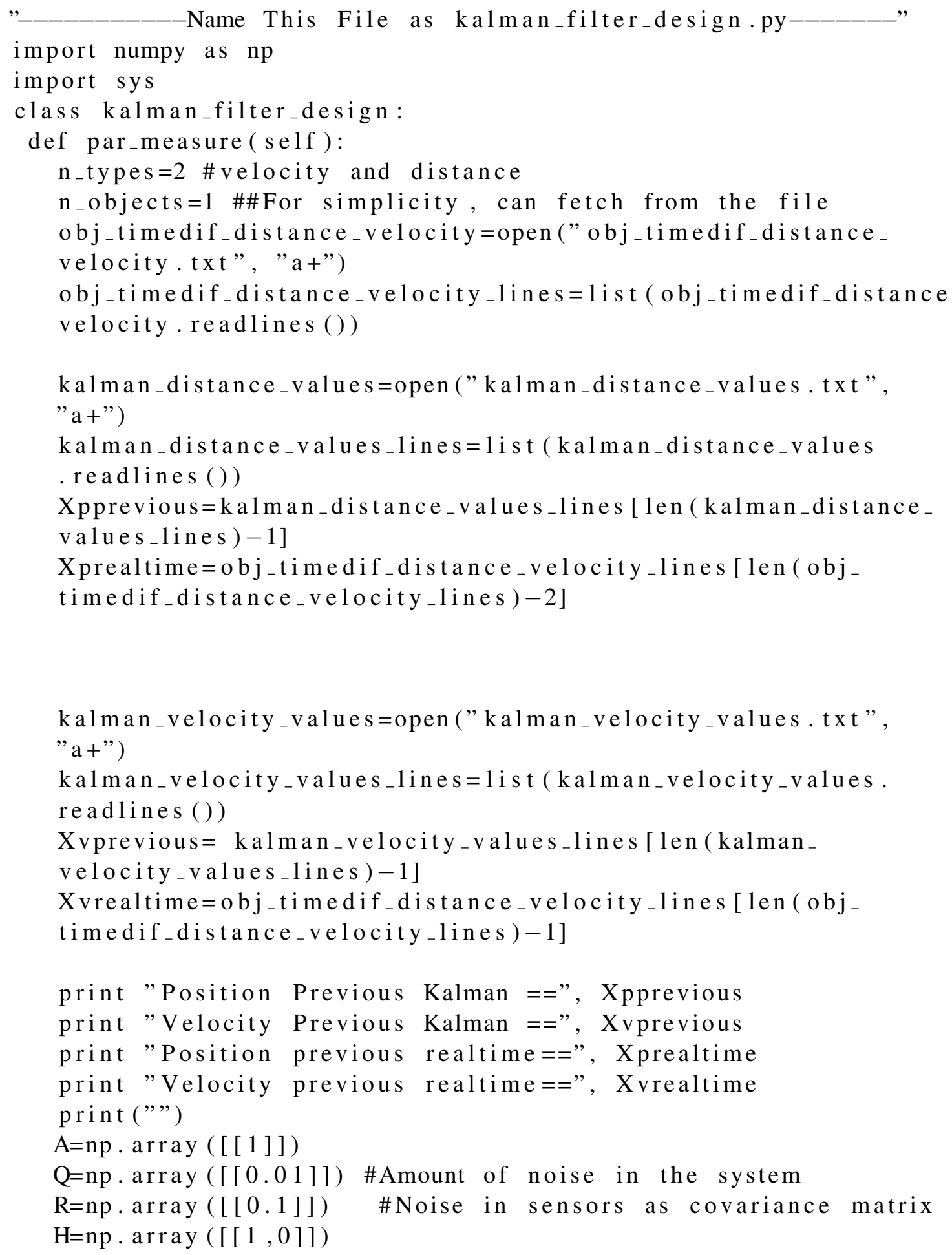


$d_{t}=o_{b_{-}}$timedif_distance _ velocity_lines [len (obj_timedif

distance_velocity_lines ) - 3 ]

Xcrealtime $=n p$. vstack $(($ float $($ Xprealtime $)$, float $($ Xvrealtime $)))$

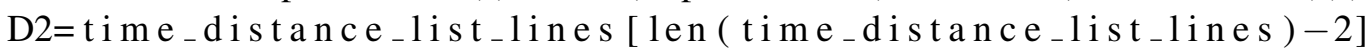

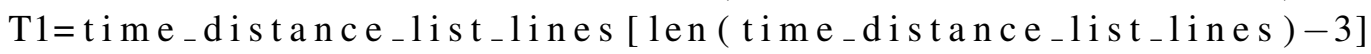

D1 = time_distance_list_lines [ len ( time_distance_list_lines )-4]

Xcprevious $=n p$. vstack $(($ float $($ Xpprevious $)$, float $($ Xvprevious $)))$

Xcpredict $=n p$. dot (np. array ([ [ float (A), float (dt)], [ float (0),

float (1)]]), np. array (Xcprevious))

Pprevious $=n p$. array $([[1,0],[0,1.96]])$

Ppredict=np. multiply (A, np. multiply (Pprevious, np.transpose (A)))

$+\mathrm{Q}$

Yinv $=\mathrm{Xcrealtime}-\mathrm{np} \cdot \operatorname{dot}(\mathrm{H}, \mathrm{Xcpredict})$

Yinv_co=np.dot $(H, n p \cdot d o t($ Ppredict , np.transpose $(H)))+R$

kf $f_{-}$ain $=n p \cdot \operatorname{dot}($ Ppredict,$n p \cdot d o t(n p \cdot t r a n s p o s e(H), n p \cdot l i n a l g$.

inv ( Yinv_co)) )

Xupdate $=$ Xcpredict + np. multiply $\left(\mathrm{kf}_{-}\right.$gain, Yinv $)$

Pupdate $=n p . d o t((n p . i d e n t i t y($ Ppredict . shape [0] $)-n p \cdot d o t(k f$ gain,$H))$

, Ppredict)

Xpupdate, Xvupdate=np.split (Xupdate, n_types)

print "Position update after Kalman =", np. savetxt(sys.stdout,

Xpupdate, $\mathrm{fmt}=\%$. $7 \mathrm{f} ")$

np.savetxt(kalman_distance_values, Xpupdate, $f m t=" \% .7 \mathrm{f}$ ")

kalman_distance_values. close ()

print "Velocity update after Kalman =", np. savetxt(sys.stdout, abs (Xvupdate), $\mathrm{fmt}=$ \%.7f $"$ )

np. savetxt (kalman_velocity_values, abs (Xvupdate), $f m t=" \% .7 f$ ")

kalman_velocity_values.close()

return

kalman_filter_design=kalman_filter_design()

kalman_filter_design.par_measure ()

sys.exit

End of kalman_filter_design.py 


\section{APPENDIX A7 \\ ACCESS POINT LOCALIZATION \\ Normal Velocity Update Python Code}

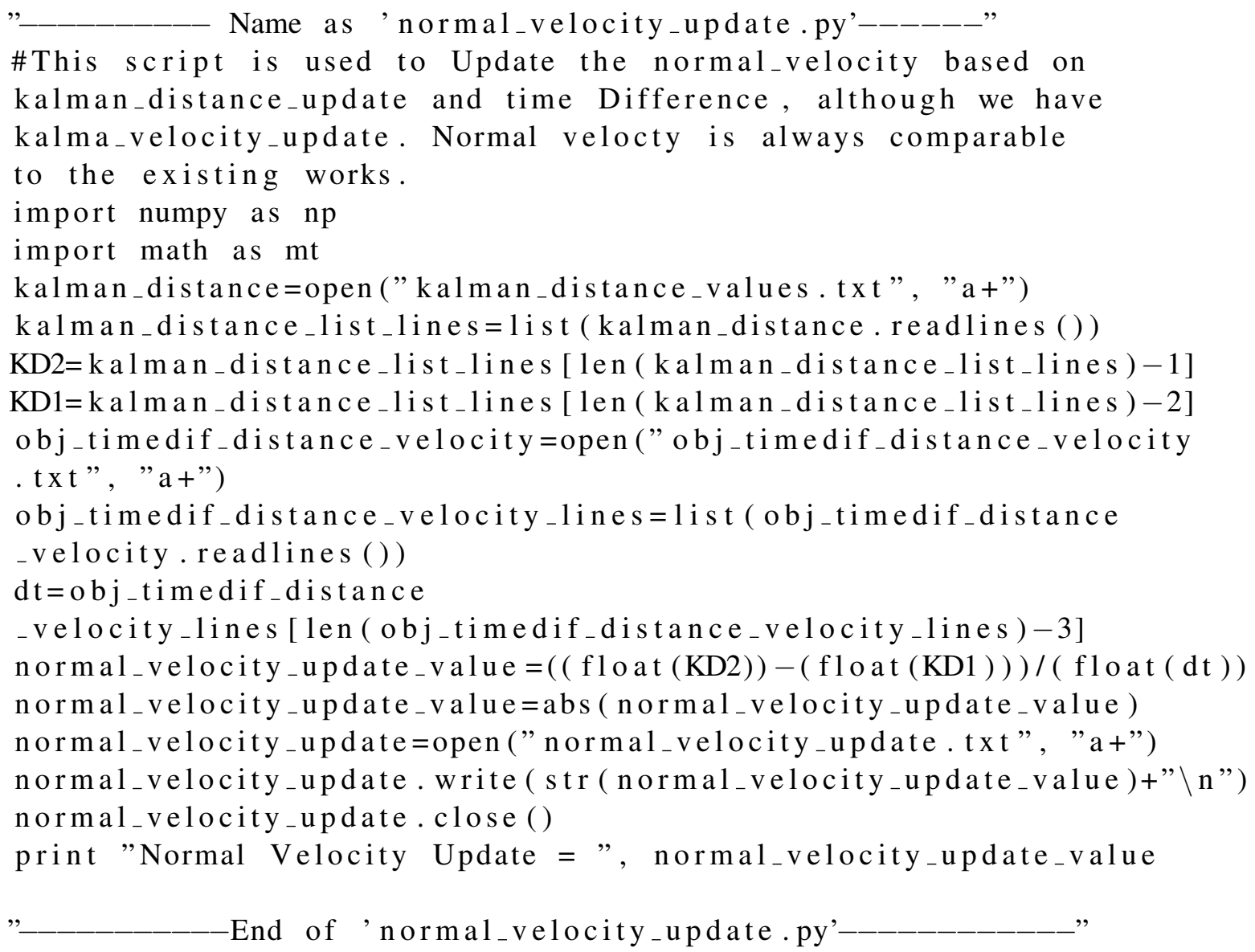

Note: All the scripts in Appendix A1 to Appendix A7 are customized exclusively for this Thesis and are mostly interdependent on each other. So, it is advisable to have all the scripts when executing the other. 


\section{APPENDIX B1}

\section{INSTALLATION}

\section{Bash Shell Script}

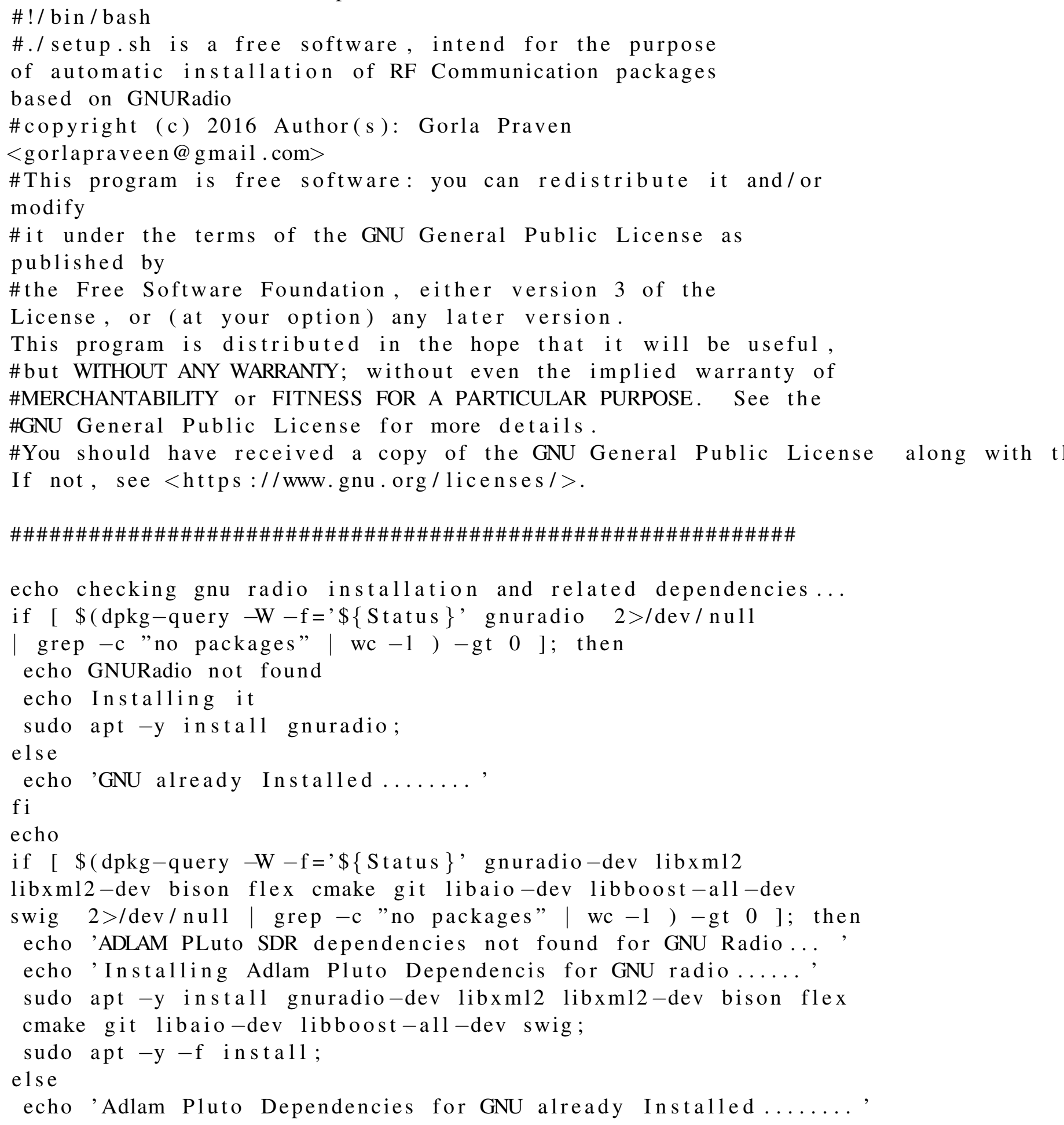


fi

echo

if $[\$($ dpkg-query $-\mathrm{W}-\mathrm{f}=$ ' $\$\{$ Status $\}$ ' libaio-dev libusb-1.0-0-dev

libserialport-dev libxml2-dev libavahi-client-dev autoconf

$2>/$ dev/nul

1 | grep -c "no packages" | wc -1$)-$ gt 0 ]; then

echo SerialCommunication packages...

echo Installing them....

sudo apt $-y$ install libaio-dev libusb-1.0-0-dev

libserialport-dev libxml2-dev libavahi-client-dev autoconf;

sudo apt $-\mathrm{y}-\mathrm{f}$ install;

else

echo 'SerialCommunication packages Already Installed'

fi

echo \#\#\#\#\#\#\#\#\#\#\#\#\#\#\#

if [ $\$(\mathrm{dpkg} \text {-query }-\mathrm{W}-\mathrm{f}=' \$\{\mathrm{Status}\}]^{\prime}$ sigrok $*$ libserialport0

libserialport-dev 2>/dev/null | grep -c "no packages"

( wc -1 ) $-\mathrm{gt} 0$ ]; then

echo Other SerialCommunication packages...

echo Installing them....

sudo apt $-y$ install $\operatorname{sigrok} *$ libserialport0 libserialport-dev;

sudo apt $-\mathrm{y}-\mathrm{f}$ install;

else

echo 'Required SerialCommunication packages Already Installed' $\mathrm{fi}$

sudo apt $-y$ install gr-iio* libad9361* libgnuradio-iio0 libiio*;

echo

mkdir Adlam_pluto_required

bash -c "cd Adlam_pluto_required \&\& git clone https://github.com/ analogdevicesinc/libiio.git gr-libiio"

bash -c "cd Adlam_pluto_required/gr-libiio/ \&\& cmake ."

bash -c "cd Adlam_pluto_required/gr-libiio/ \&\& make"

bash -c "cd Adlam_pluto_required/gr-libiio/ \&\& sudo make install"

bash -c "cd Adlam_pluto_required \&\& git clone https://github.com/ analogdevicesinc/libad9361-iio.git gr-libad9361-iio"

bash -c "cd Adlam_pluto_required/gr-libad9361-iio/ \&\& cmake ." bash -c "cd Adlam_pluto_required/gr-libad9361-iio/ \&\& make" bash -c "cd Adlam_pluto_required/gr-libad9361-iio/ \&\& sudo make install" 
bash -c "cd Adlam_pluto_required \&\& git clone https://github.com/ analogdevicesinc/gr-iio.git gr-iio"

bash -c "cd Adlam_pluto_required/gr-iio/ \&\& cmake

-DCMAKE_INSTALL_PREFIX =/ u s r ."

bash -c "cd Adlam_pluto_required/gr-iio/ \&\& make"

bash -c "cd Adlam_pluto_required/gr-iio/ $\& \&$ sudo make install"

bash -c "sudo ldconfig"

bash -c"cp-r / usr/local/lib/python2.7/dist-packages/gnuradio/

iio / usr/lib/python2.7/dist-packages/gnuradio/"

echo

echo

echo Installation of Adlam pltuo is completed

echo

echo

echo Checking Installtion of IEEEE802.11 GNU radio based libraries.

echo Installing required packages

sudo apt-get install liblog $4 \mathrm{cpp} 5-$ dev

bash $-c$ "cd Adlam_pluto_required \&\&

git clone https://github.com/bastibl/gr-foo.git gr-foo"

bash -c "cd Adlam_pluto_required/gr-foo \&\& git checkout master"

bash -c "mkdir Adlam_pluto_required/gr-foo/build"

echo 'Installing the $\mathrm{gr}-$ foo, a meta package required for

GNU Radio IEEE802.11 Implementation,

bash -c "cd Adlam_pluto_required/gr-foo/build \&\& cmake .."

bash -c "cd Adlam_pluto_required/gr-foo/build \&\& make"

bash -c "cd Adlam_pluto_required/gr-foo/build \&\& sudo make install"

bash $-c$ "sudo 1dconfig"

echo

bash -c "cd Adlam_pluto_required \&\& git clone https://github .

com/bastibl/gr-ieee802-11.git gr-ieee802-11"

bash -c "mkdir Adlam_pluto_required/gr-ieee802-11/build"

echo 'Installing the GNU Radio IEEE802.11 Implementation'

bash -c "cd Adlam_pluto_required/gr-ieee802-11/build \&\& cmake .."

bash -c "cd Adlam_pluto_required/gr-ieee802-11/build \&\& make"

bash -c "cd Adlam_pluto_required/gr-ieee802-11/build \&\& sudo make install"

bash -c "sudo ldconfig" 
echo

echo Adjusting the Maximum Shared Memory to 2147483648

sudo sysctl - w kernel $. \operatorname{shmmax}=2147483648$

bash -c "cd ../ \&\& python wifi_phy_hier.py"

sudo mkdir $\sim$.gnuradio

echo $[$ grc] $>>\sim$ /gnuradio/config. conf

echo global_blocks path = / opt/local/share/gnuradio/grc/blocks $>$ /.gnuradio/config. conf

echo local_blocks_path = /Users/basti/usr/share/gnuradio/grc

/blocks > $>$ /gnuradio/config. conf

echo

echo Installing gfortran, lapack, itpp packages

sudo apt $-y$ install gfortran

sudo apt -y install libatlas-base-dev libblas-dev liblapack-dev

bash - c "cd ../ required/itpp - 4.3.1/ \&\& mkdir build"

bash $-c$ "cd ../ required/itpp $-4.3 .1 /$ build \&\& cmake .."

bash -c "cd ../ required/itpp - 4.3.1/build \&\& make"

bash $-c$ "cd ../ required/itpp -4.3.1/build \&\& sudo make install."

sudo apt -y install libatlas-base-dev libblas-dev liblapack-dev

sudo apt $-\mathrm{f}$ install

echo 'Installing RF-TAP, gr-rftap libraries for gnu radio' bash -c "cd Adlam_pluto_required \&\& git clone https://

github.com/rftap/gr-rftap gr-rftap"

bash -c "mkdir Adlam_pluto_required/gr-rftap/build"

echo 'Installing the gr-rftap, a meta package required for

GNU Radio IEEE802.11 Implementation'

bash -c "cd Adlam_pluto_required/gr-rftap/build \&\& cmake .."

bash -c "cd Adlam_pluto_required/gr-rftap/build \&\& make"

bash $-c$ "cd Adlam_pluto_required/gr-rftap/build \&\& sudo

make install"

bash -c "cd Adlam_pluto_required/ \&\& sudo ldconfig"

echo Installing wireshark and tshark ......

sudo apt $-\mathrm{y}$ install wireshark*

sudo apt $-\mathrm{y}$ install tshark*

sudo apt $-\mathrm{y}-\mathrm{f}$ install

echo

echo

echo 


\section{APPENDIX B2}

\section{IEEE802.11 a/g/p}

\section{Bash Shell Script}

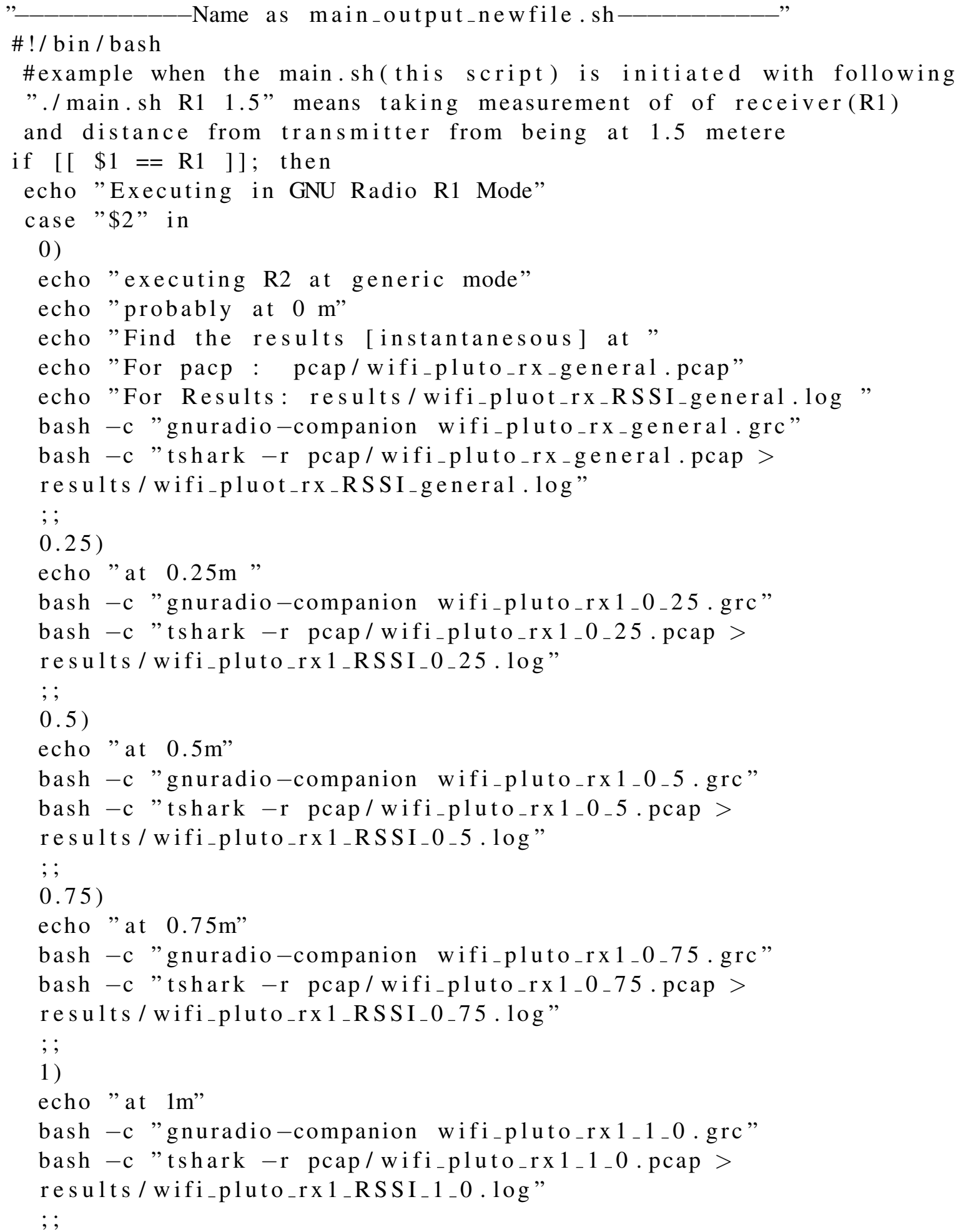




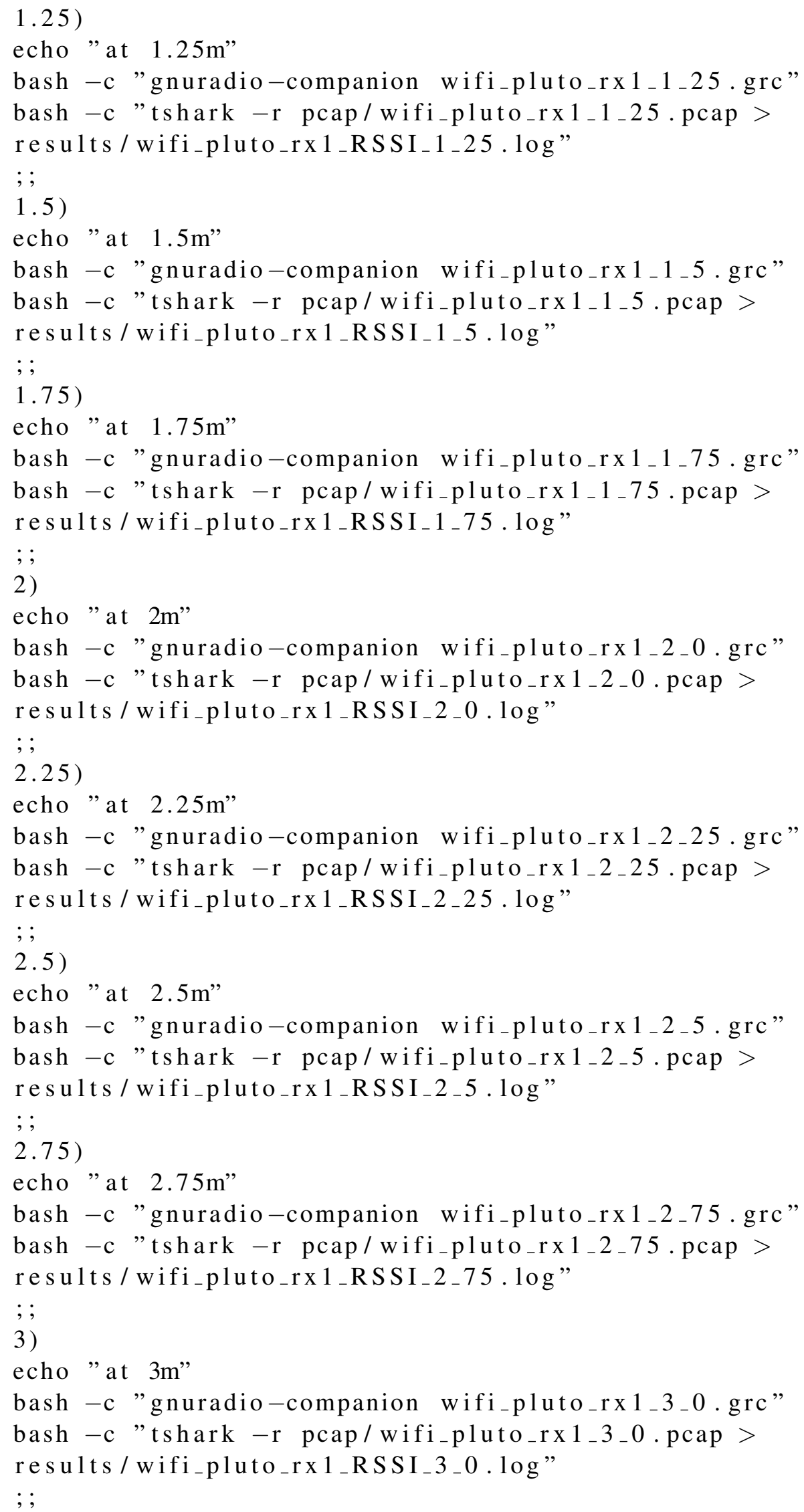




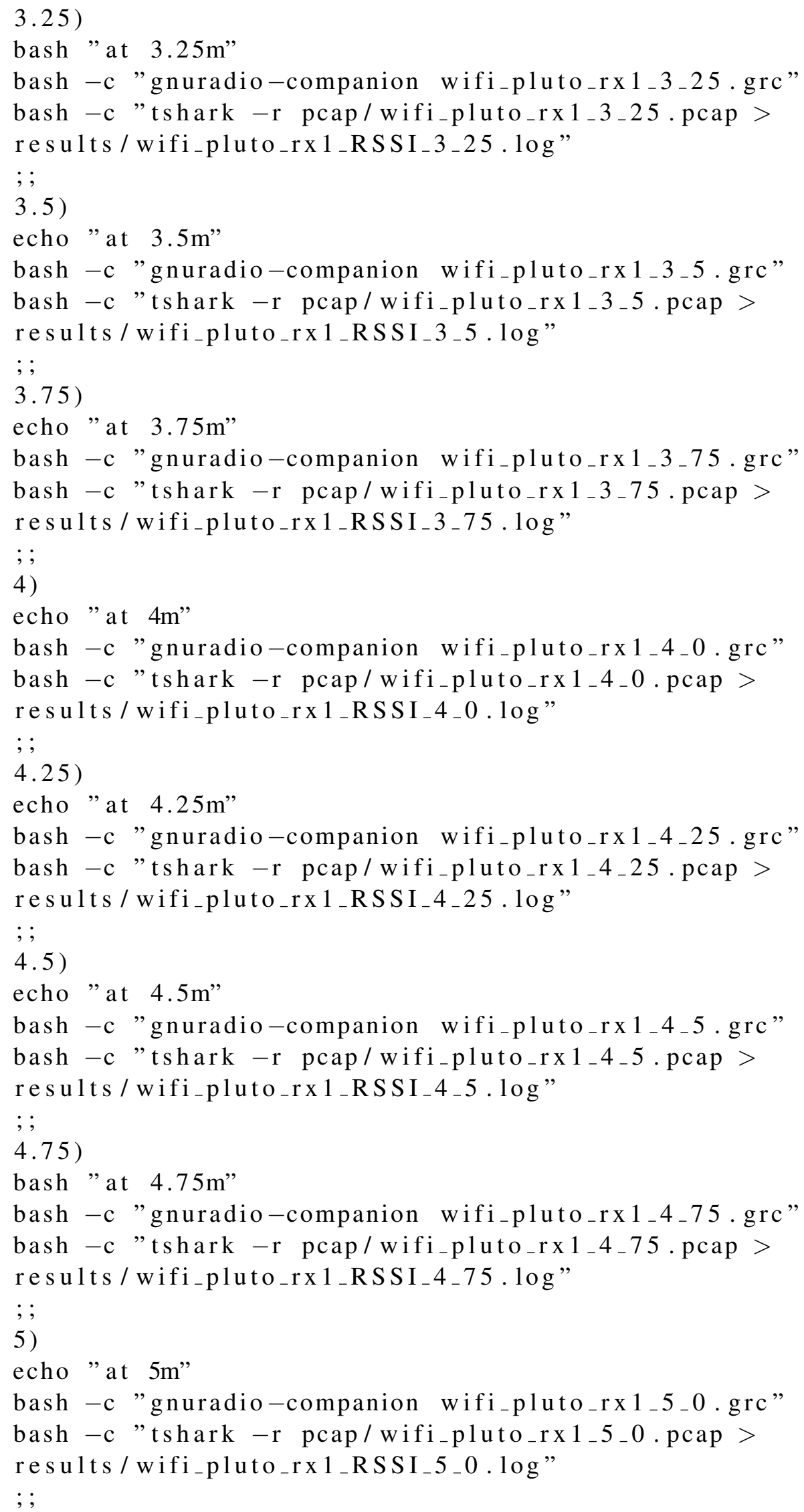




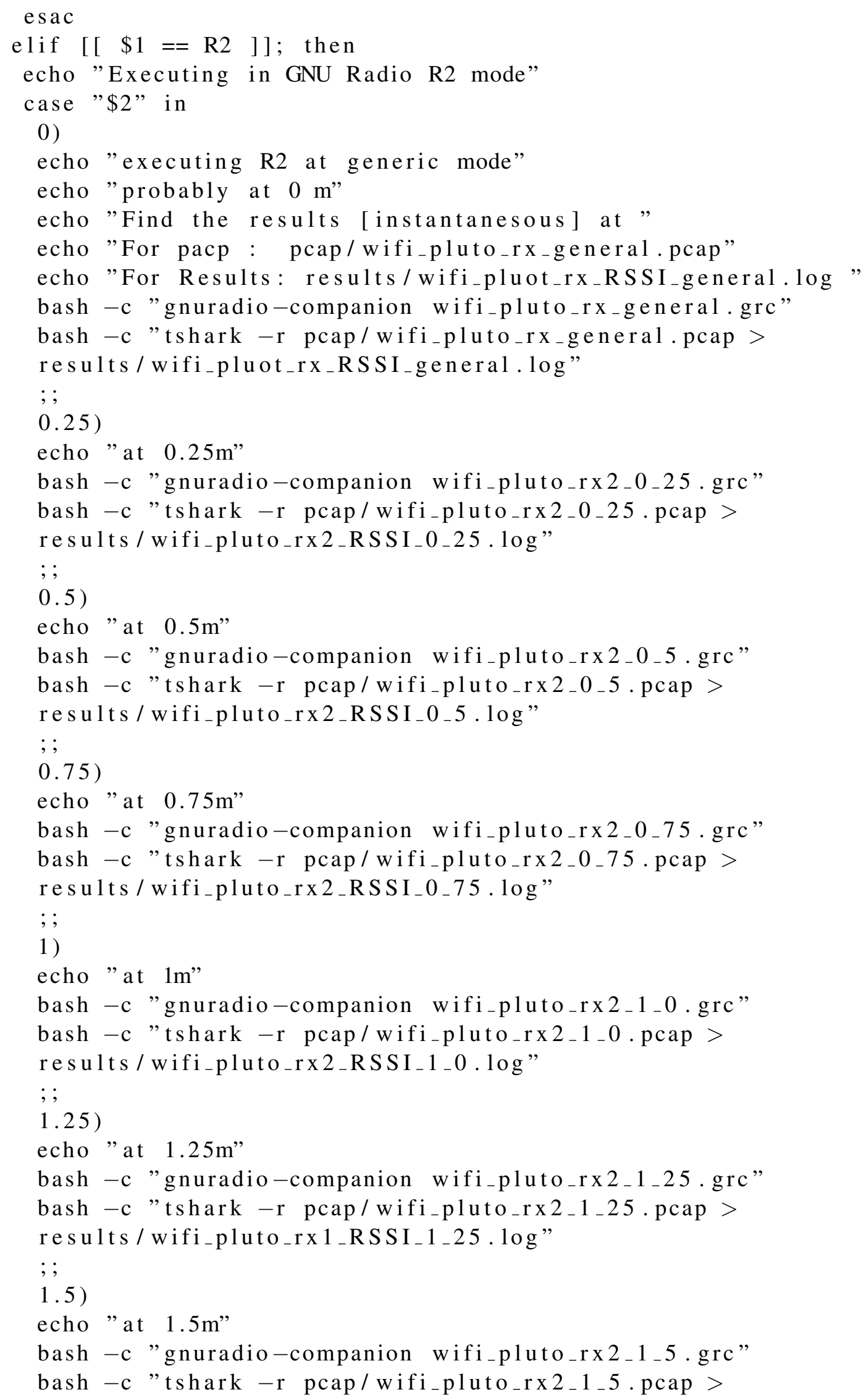




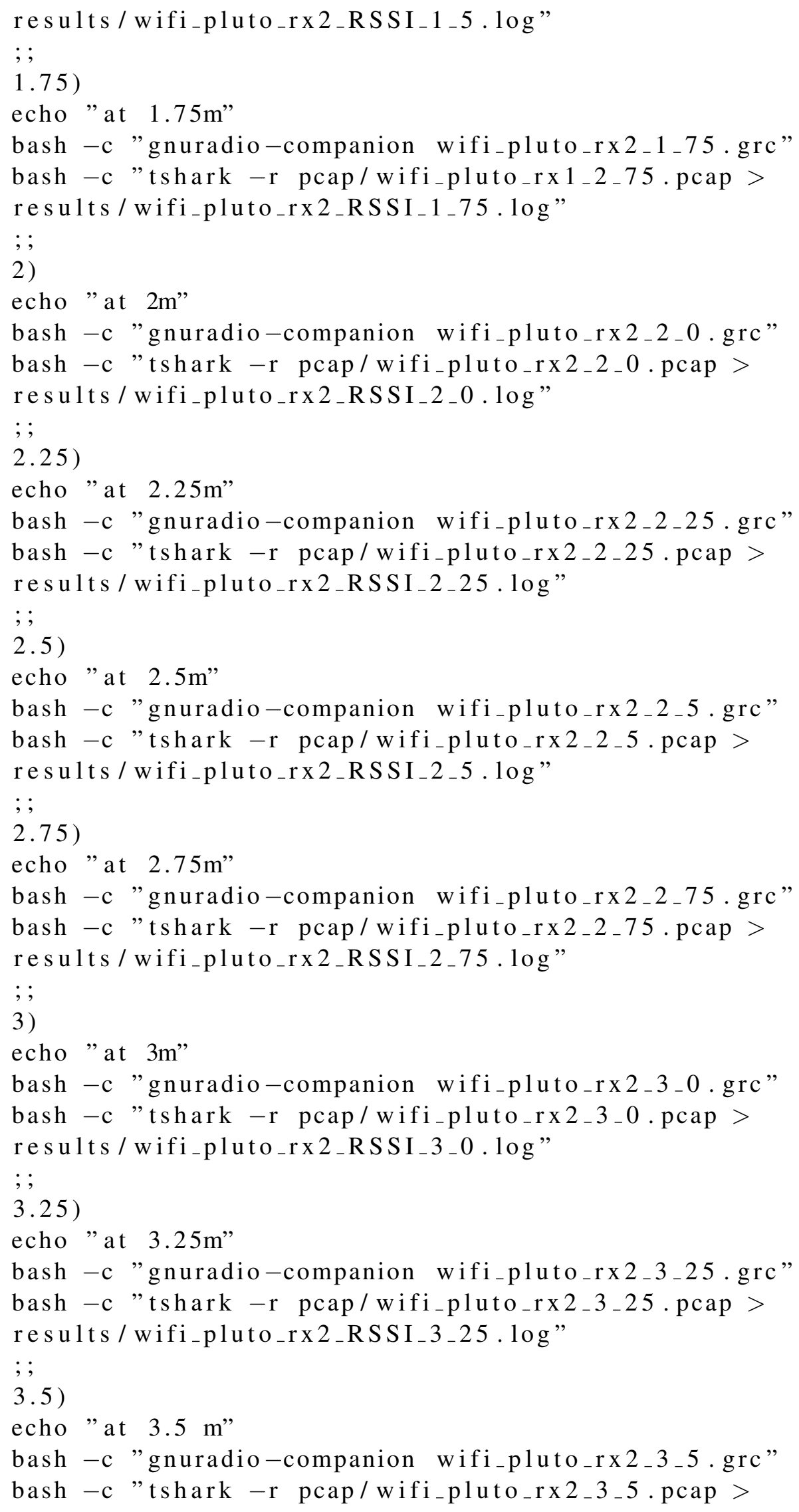




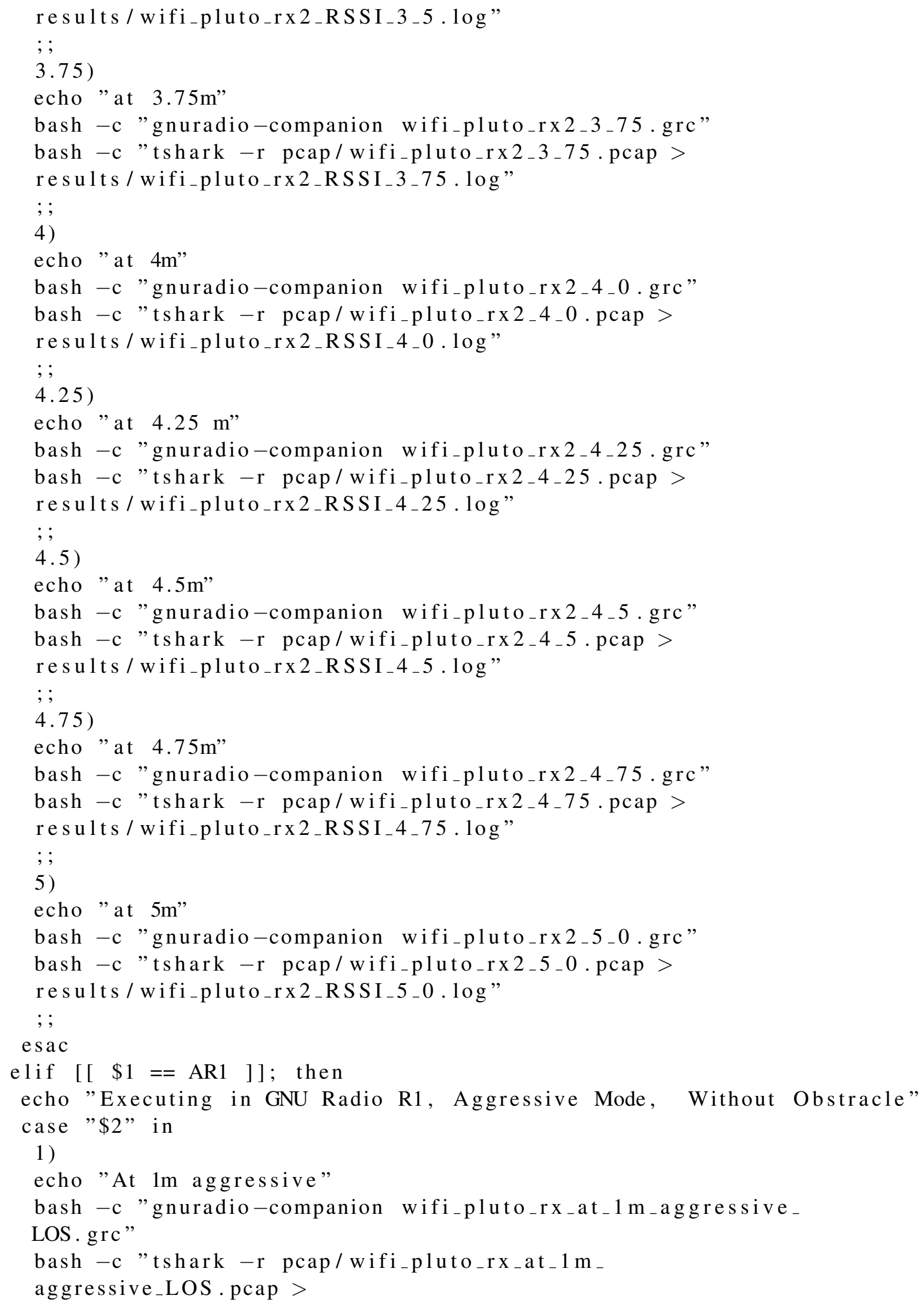




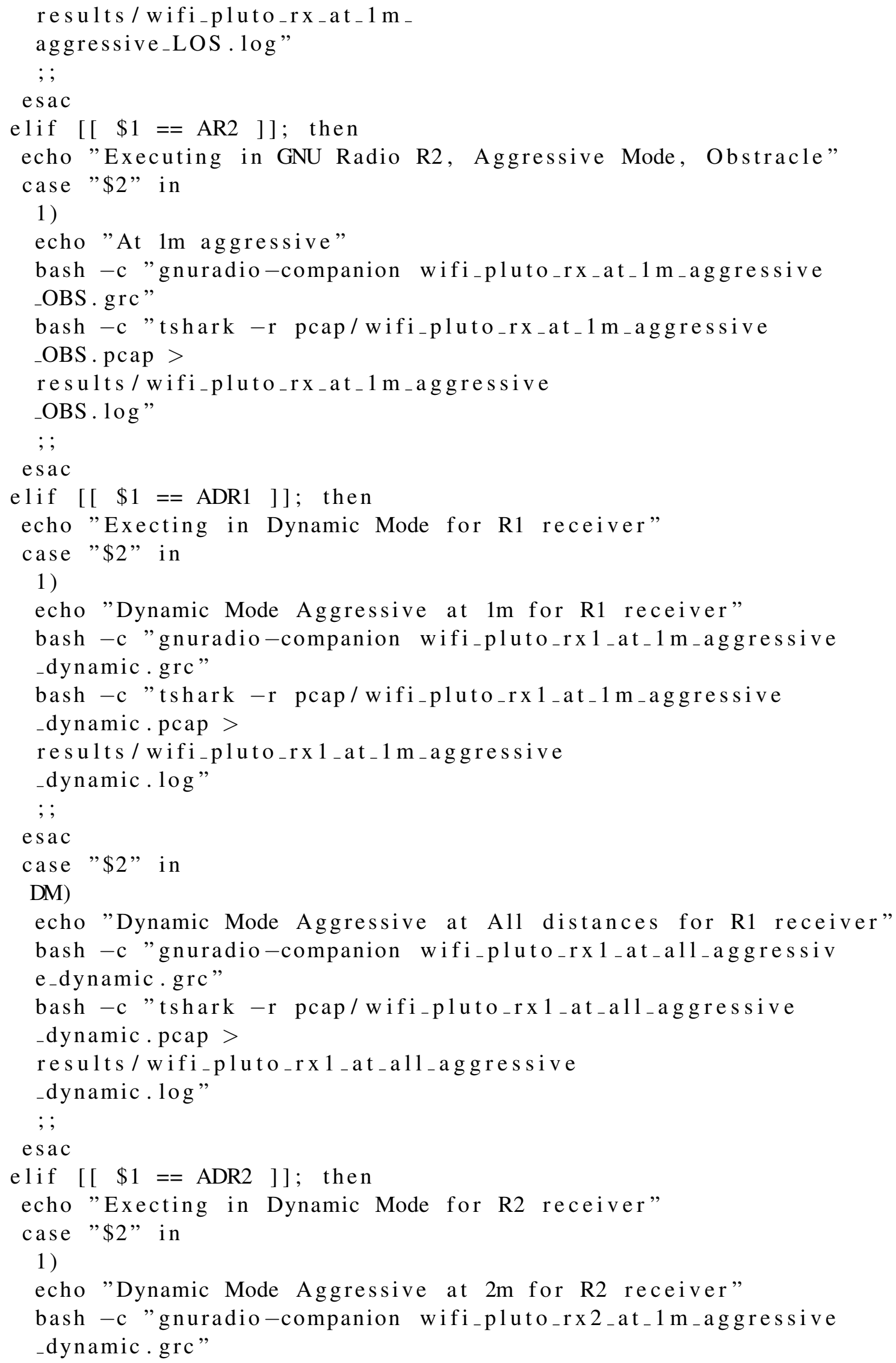




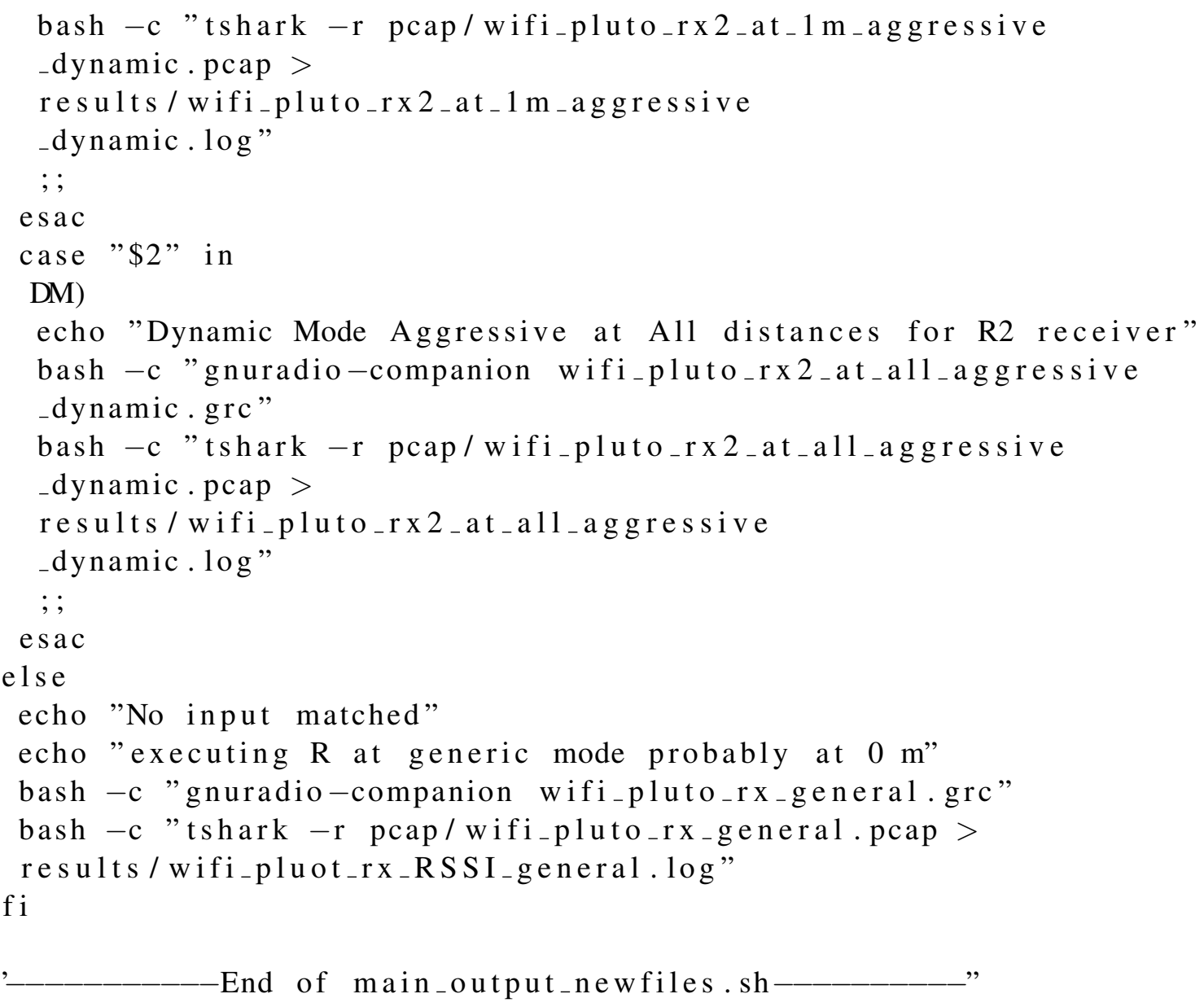




\section{APPENDIX B3}

IEEE802.11 a/g/p

Gnu Radio Python Script

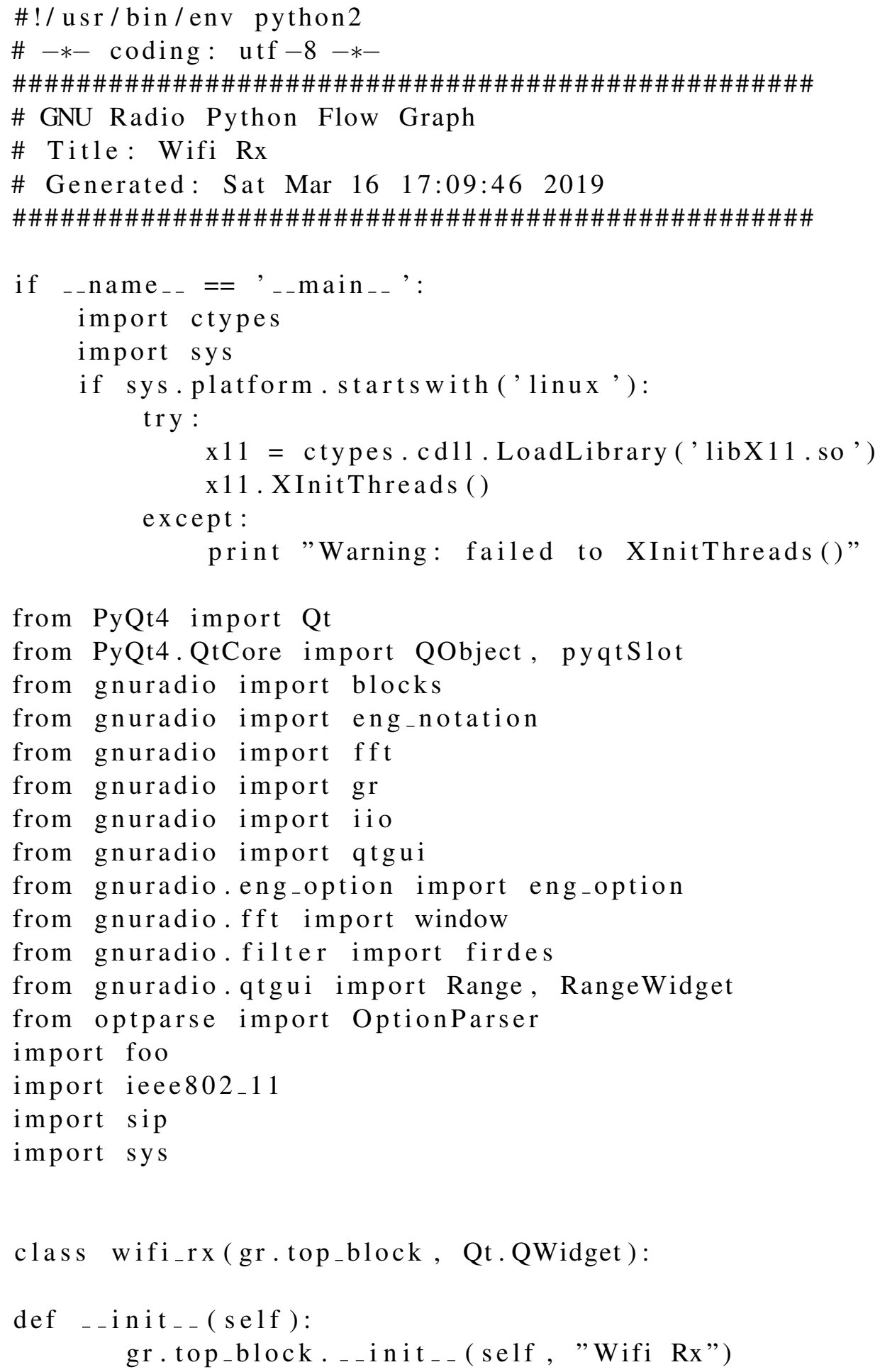




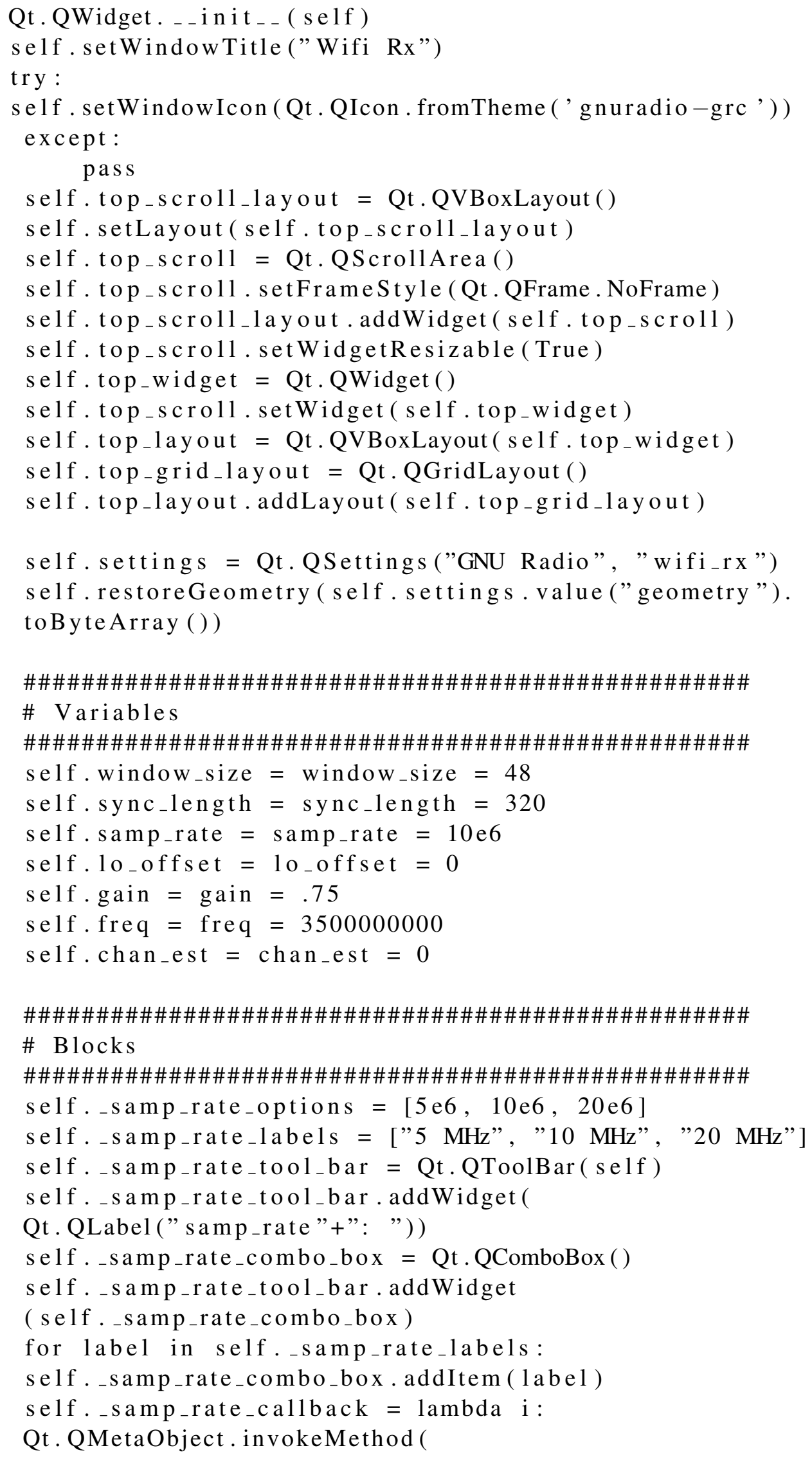


self ._samp_rate_combo_box, "setCurrentIndex", Qt.Q_ARG

("int", self ._samp_rate_options.index (i )))

self . _ samp_rate_callback (self . samp_rate)

self ._samp_rate_combo_box . currentIndex Changed . connect (

lambda i : self.

set_samp_rate (self__samp_rate_options [i ]))

self . top_layout addWidget (self . _samp_rate_tool_bar)

self._freq_options = [2412000000.0, 2417000000.0,

$2422000000.0,2427000000.0$,

$2432000000.0,2437000000.0,2442000000.0$,

$2447000000.0,2452000000.0,2457000000.0$,

$2462000000.0,2467000000.0,2472000000.0$,

$2484000000.0,5170000000.0,5180000000.0$,

5190000000.0 , 5200000000.0, 5210000000.0,

5220000000.0 , 5230000000.0, 5240000000.0,

5250000000.0 , 5260000000.0, 5270000000.0,

5280000000.0 , 5290000000.0, 5300000000.0,

5310000000.0 , 5320000000.0, 5500000000.0,

$5510000000.0,5520000000.0,5530000000.0$,

5540000000.0 , 5550000000.0, 5560000000.0,

$5570000000.0,5580000000.0,5590000000.0$,

5600000000.0 , 5610000000.0, 5620000000.0,

5630000000.0 , 5640000000.0, 5660000000.0,

$5670000000.0,5680000000.0,5690000000.0$,

$5700000000.0,5710000000.0,5720000000.0$,

$5745000000.0,5755000000.0,5765000000.0$,

$5775000000.0,5785000000.0,5795000000.0$,

$5805000000.0,5825000000.0,5860000000.0$,

$5870000000.0,5880000000.0,5890000000.0$,

5900000000.0 , 5910000000.0, 5920000000.0,

$3500000000.0]$

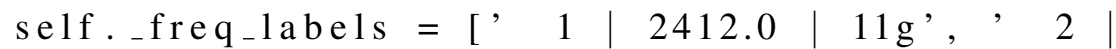

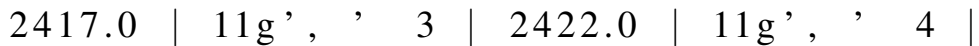

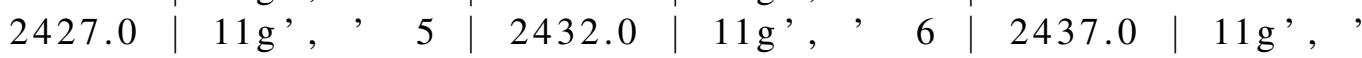

$2442.0|11 \mathrm{~g},, \quad, \quad 8| 2447.0 \mid 11 \mathrm{~g},, \quad, \quad 9$

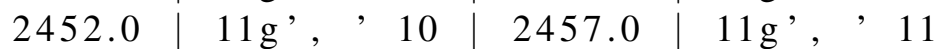

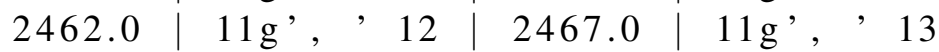

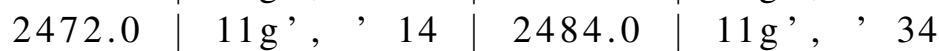

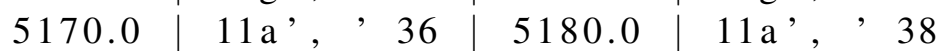

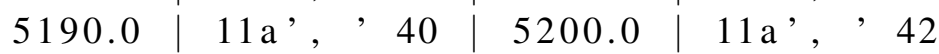

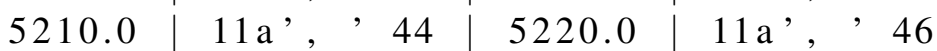

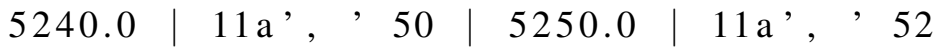

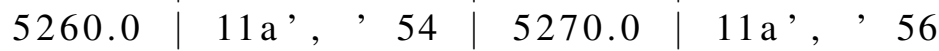

$5280.0|11 \mathrm{a},,, 58| 5290.0 \mid 11 \mathrm{a},, \quad, 60$

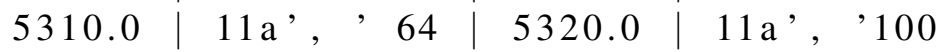

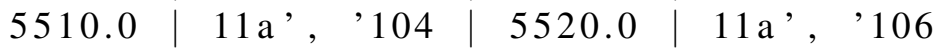

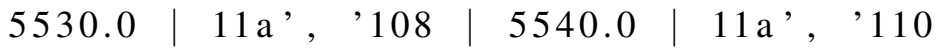

$5230.0 \mid 11 \mathrm{a}, \quad, \quad 48$

$5300.0 \mid 11 \mathrm{a},, \quad 62$

5500.0 | $11 \mathrm{a},,, 102$ 


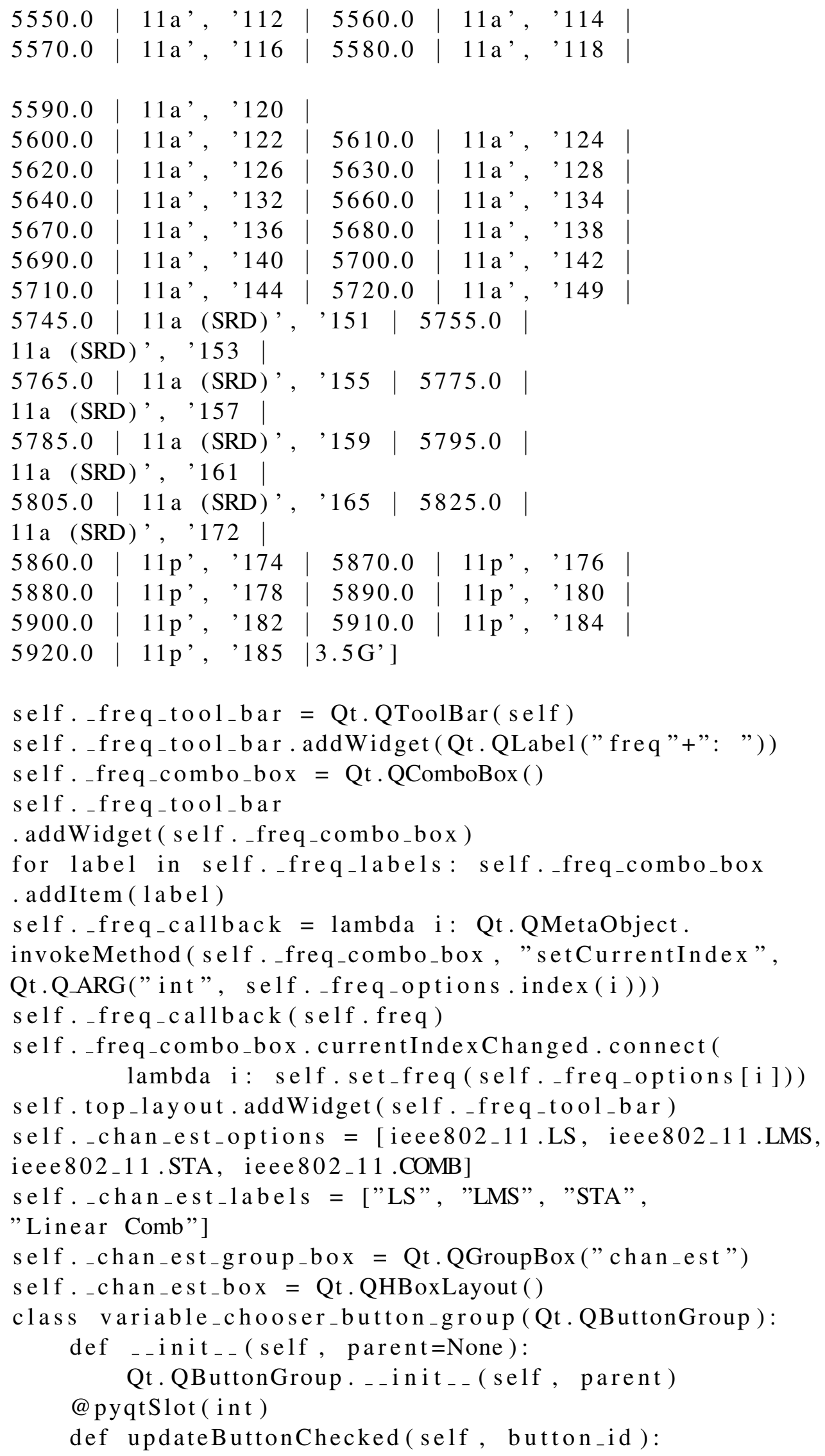




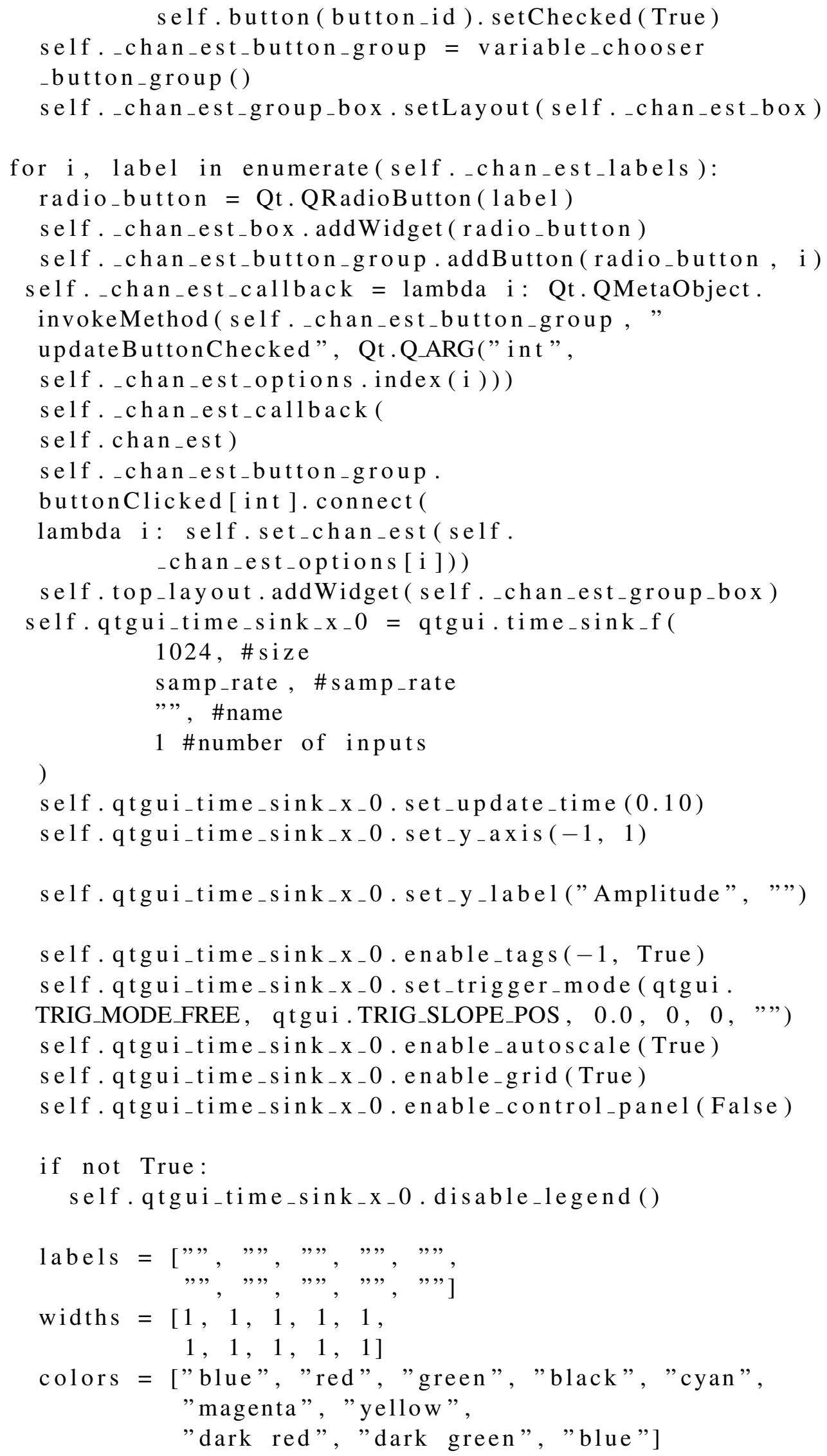




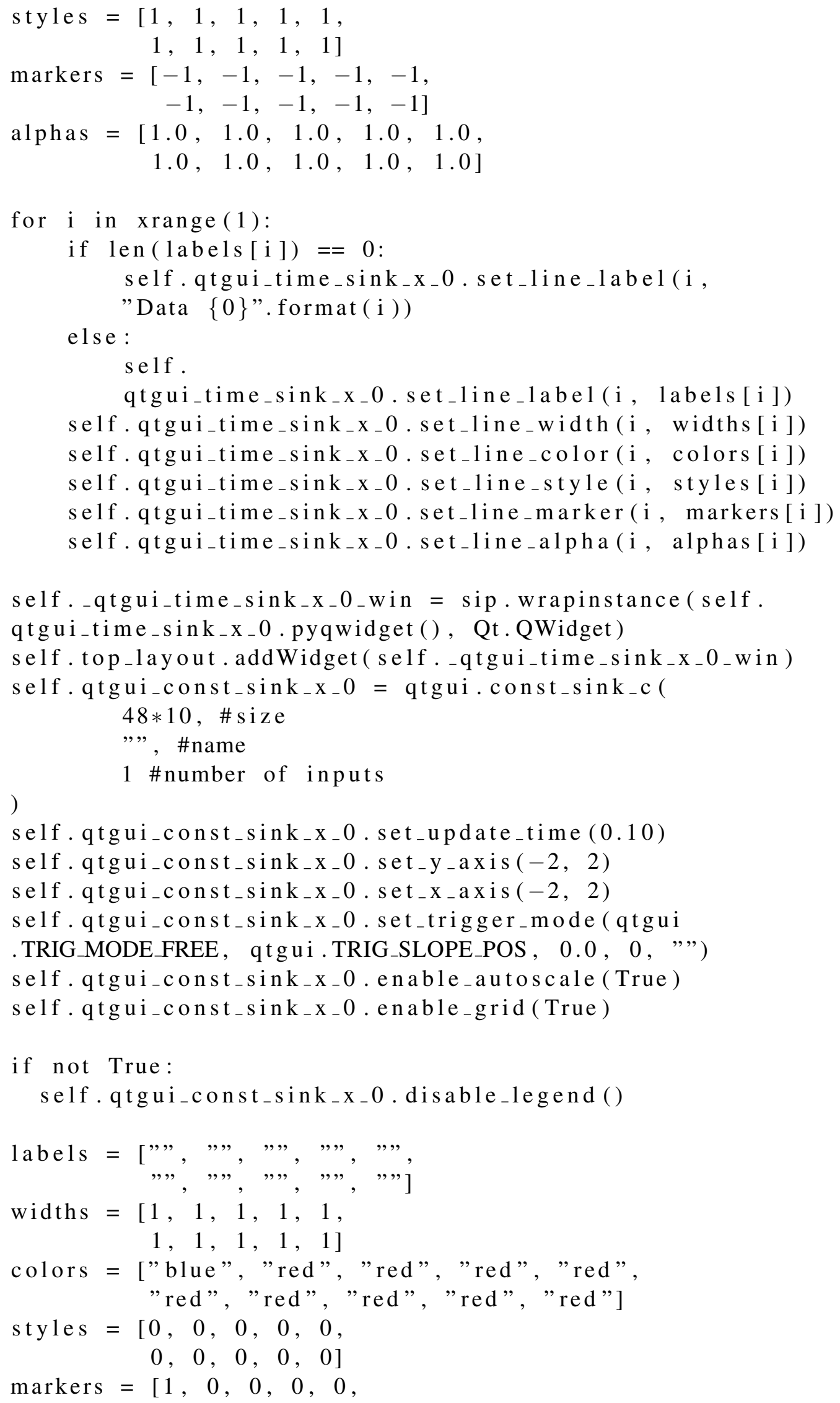




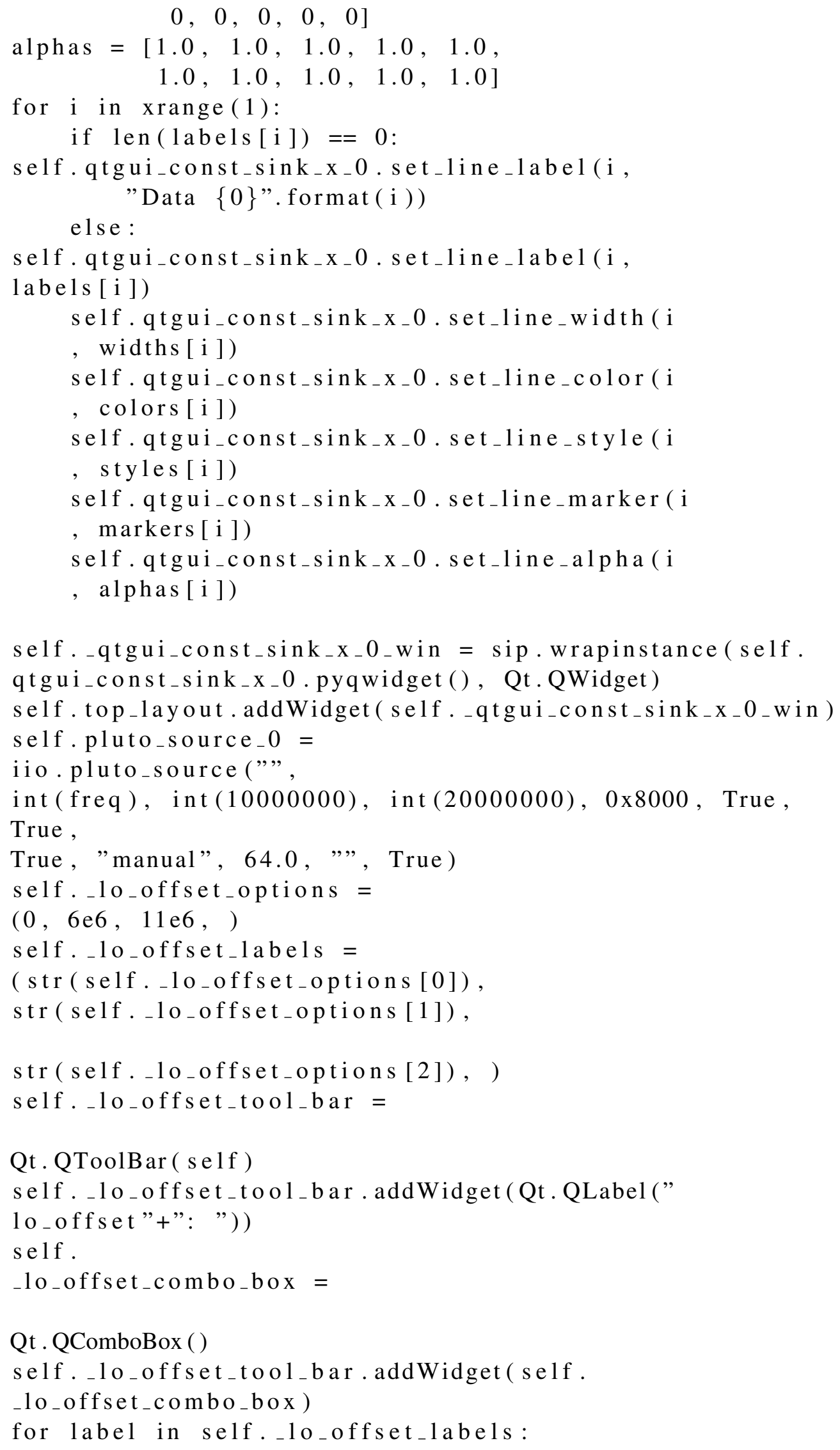




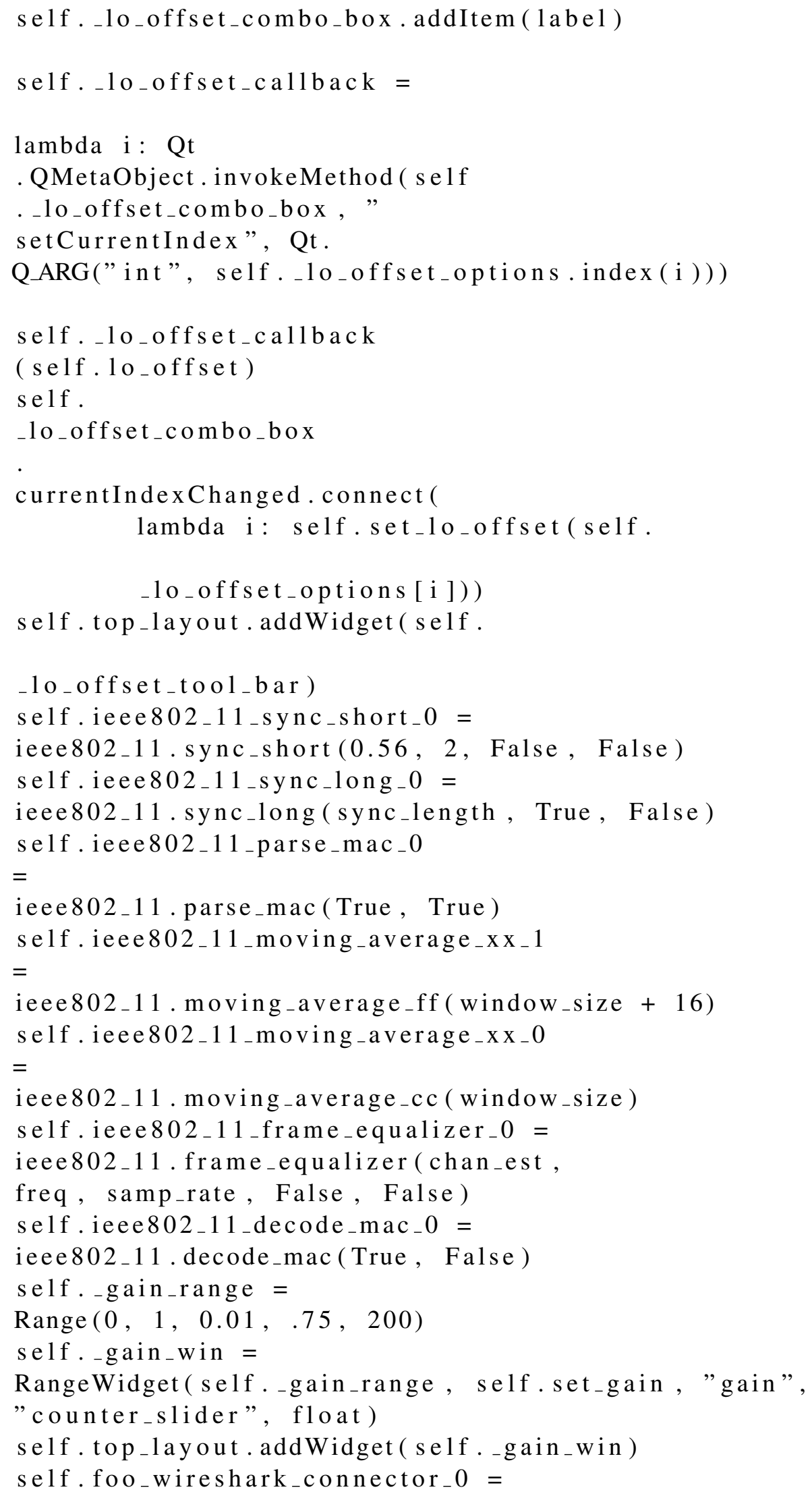


foo. wireshark_connector (127, True)

self. $\mathrm{fft}_{-} \mathrm{vxx}_{-} 0=$

fft.fft_vcc (64, True, (window.rectangular (64)), True, 1)

self.blocks_stream_to_vector $0=$

blocks.stream_to_vector ( $g r$. sizeof_gr_complex $* 1,64)$

self.blocks_pdu_to_tagged_stream_1 =

blocks.pdu_to_tagged_stream (blocks . complex_t, "packet_len")

self. blocks_multiply_- $x_{-} 0=$

blocks.multiply_vcc (1)

self.blocks_file_sink_0

$=$ blocks.file sink $_{\text {gr. }}$ sizeof $c h a r * 1$,

"/home/ praveen-ubuntu-radio / Documents / Gnu_radio /

Gnu_radio_programs/ieee802_11_Radio_Localization/Original /

pcap/wifi_pluto_rx2_at_all_aggressive_dynamic.pcap”, True)

self.blocks_file_sink_0.set_unbuffered (True)

self.blocks_divide $\mathrm{xx}_{-} 0$

$=$ blocks.divide $_{-} f(1)$

self.blocks_delay $0_{-} 0$

$=$ blocks.delay $\left(\mathrm{gr} . \operatorname{sizeof}_{-} \mathrm{gr}_{-} \operatorname{complex} * 1,16\right)$

self.blocks_delay 0

$=$ blocks.delay $\left(\mathrm{gr}\right.$. sizeof $_{-} \mathrm{gr}_{-} \mathrm{complex}_{*} 1$, sync_length $)$

self.blocks_conjugate cc $_{-} 0$

$=$ blocks.conjugate $\_c()$

self . blocks_complex_to_mag_squared_0

= blocks.complex_to_mag_squared (1)

self.blocks_complex_to_mag_0

$=$ blocks.complex_to_mag (1)

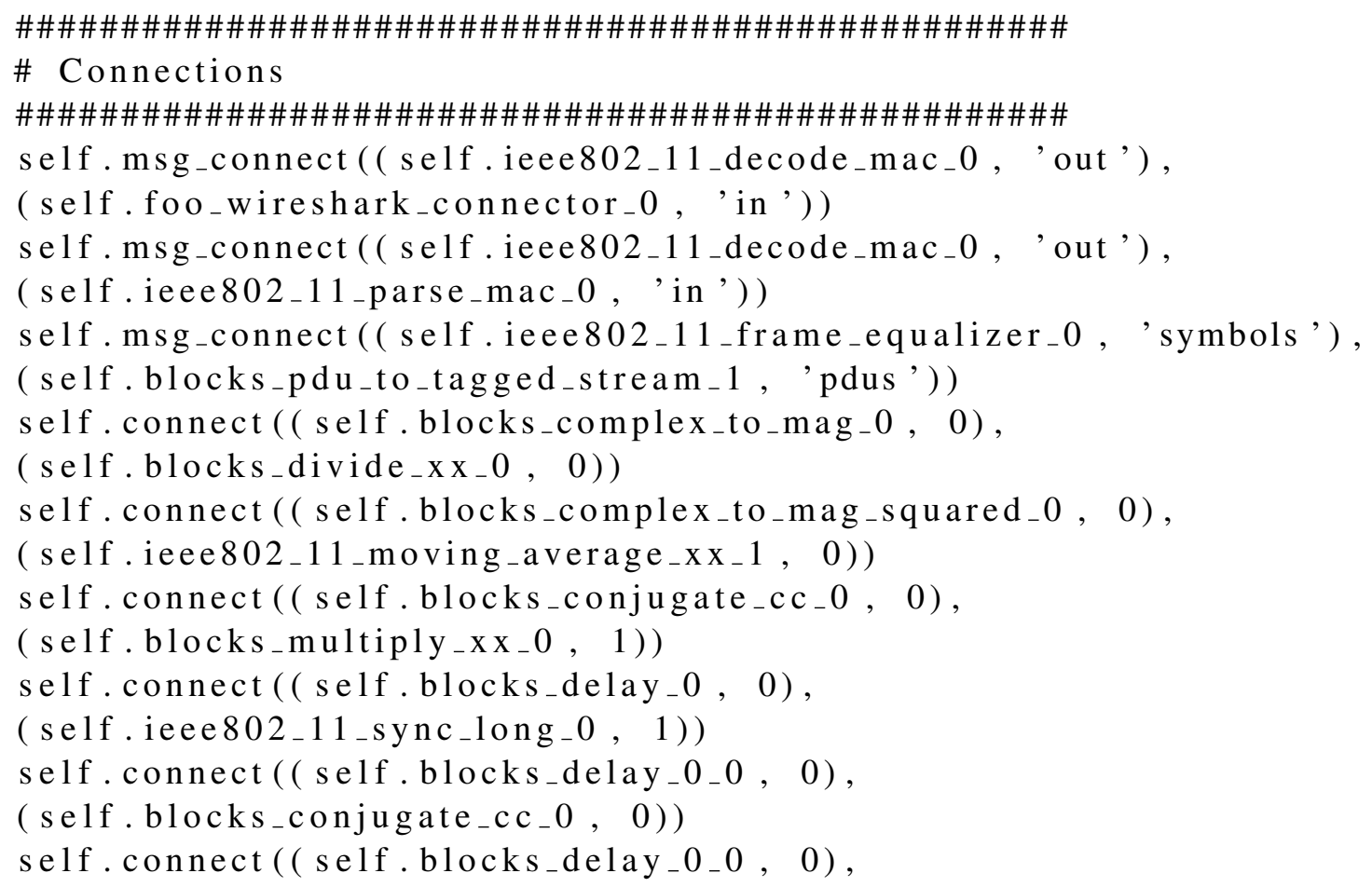


( self . ieee802_11_sync_short_o, 0$)$ )

self . connect ( ( self.blocks_divide $\left.x_{-} x_{-} 0,0\right)$,

( self . ieee802_11_sync_short_0, 2))

self. connect ( ( self.blocks_divide $\left.x_{-} x_{-} 0,0\right)$,

( self.qtgui_time_sink $\left.\mathrm{x}_{-} 0,0\right)$ )

self . connect ( ( self.blocks_multiply_ $\left.x_{-} 0,0\right)$,

( self . ieee802_11_moving average _ $\left._{-} x_{-} 0,0\right)$ )

self.connect ( ( self.blocks _pdu_to_tagged_stream_1, 0),

( self.qtgui const_sink $\left.\mathrm{k}_{-} \mathrm{x}_{-} 0,0\right)$ )

self.connect ( ( self.blocks_stream_to_vector 0,0$)$,

$\left(\mathrm{self} . \mathrm{fft}_{-} \mathrm{vxx}_{-} 0,0\right)$ )

self. connect ( ( self. fft $\left.\mathrm{vxx}_{-} 0,0\right)$,

( self .ieee802_11_frame_equalizer 0,0$)$ )

self. connect ( ( self.foo_wireshark_connector_o, 0$)$,

( self.blocks_file sink $\left._{-} 0,0\right)$ )

self. connect ( ( self . ieee802_11_frame_equalizer_o, 0$)$,

( self . ieee802_11_decode_mac 0,0$)$ )

self. connect ( ( self. ieee802_11 _moving average $\left.x_{-} x_{-} 0,0\right)$,

( self.blocks_complex_to_mag_ 0,0$)$ )

self. connect ( ( self. ieee802_11_moving average $\left.x_{-} x_{-} 0,0\right)$,

( self . ieee802_1 $11_{-}$sync $_{-}$short $\left._{-} 0,1\right)$ )

self . connect ( (self. ieee802_11_moving average $\left.x_{-} x_{-} 1,0\right)$,

( self.blocks_divide $\left.x_{-} x_{-} 0,1\right)$ )

self. connect ( ( self . ieee802_1 _ sync_long_0, 0$)$,

( self .blocks_stream_to_vector_ 0,0$)$ )

self. connect ( (self. ieee802_11_sync short_o, 0$)$,

( self .blocks_delay_0, 0$)$ )

self . connect ( (self. ieee802_11 _sync_short_0, 0$)$,

( self . ieee802_11_sync_long_0, 0$)$ )

self.connect ( ( self.pluto_source_0, 0$)$,

( self.blocks_complex_to_mag_squared_0, 0$)$ )

self.connect ( ( self.pluto_source 0,0$)$,

$($ self.blocks_delay_0_0, 0$)$ )

self. connect ( ( self . pluto_source 0,0$)$,

( self.blocks_multiply_x $\left.x_{-} 0,0\right)$ )

def closeEvent(self, event):

self. settings = Qt. QSettings ("GNU Radio", "wifi_rx")

self. settings. setValue ("geometry", self . saveGeometry ())

event. accept ()

def get_window_size(self):

return self. window_size

def set_window_size( self, window_size):

self. window_size $=w_{\text {indow_size }}$

self . ieee802_1 _ moving _average_x x_0 . set_length ( 


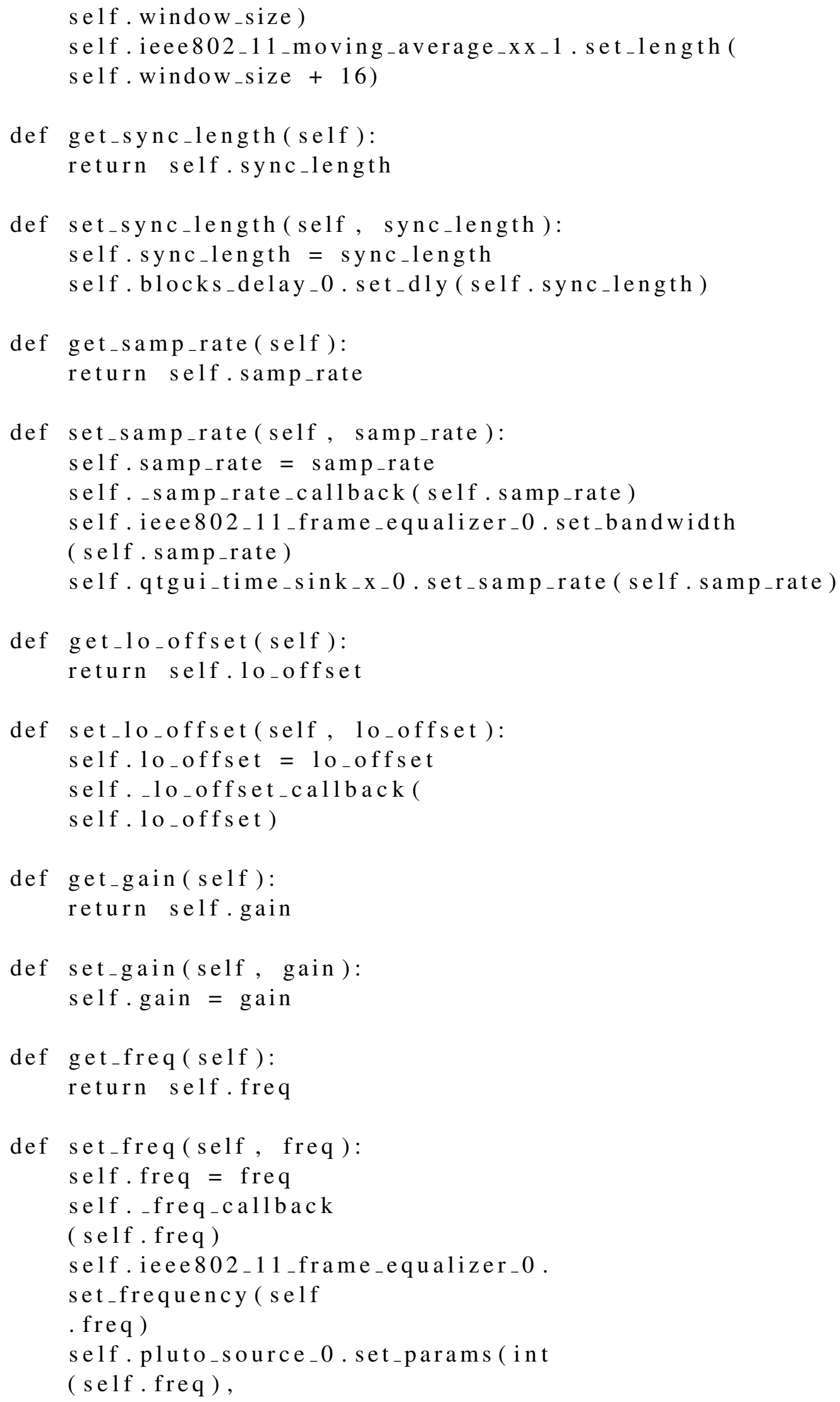




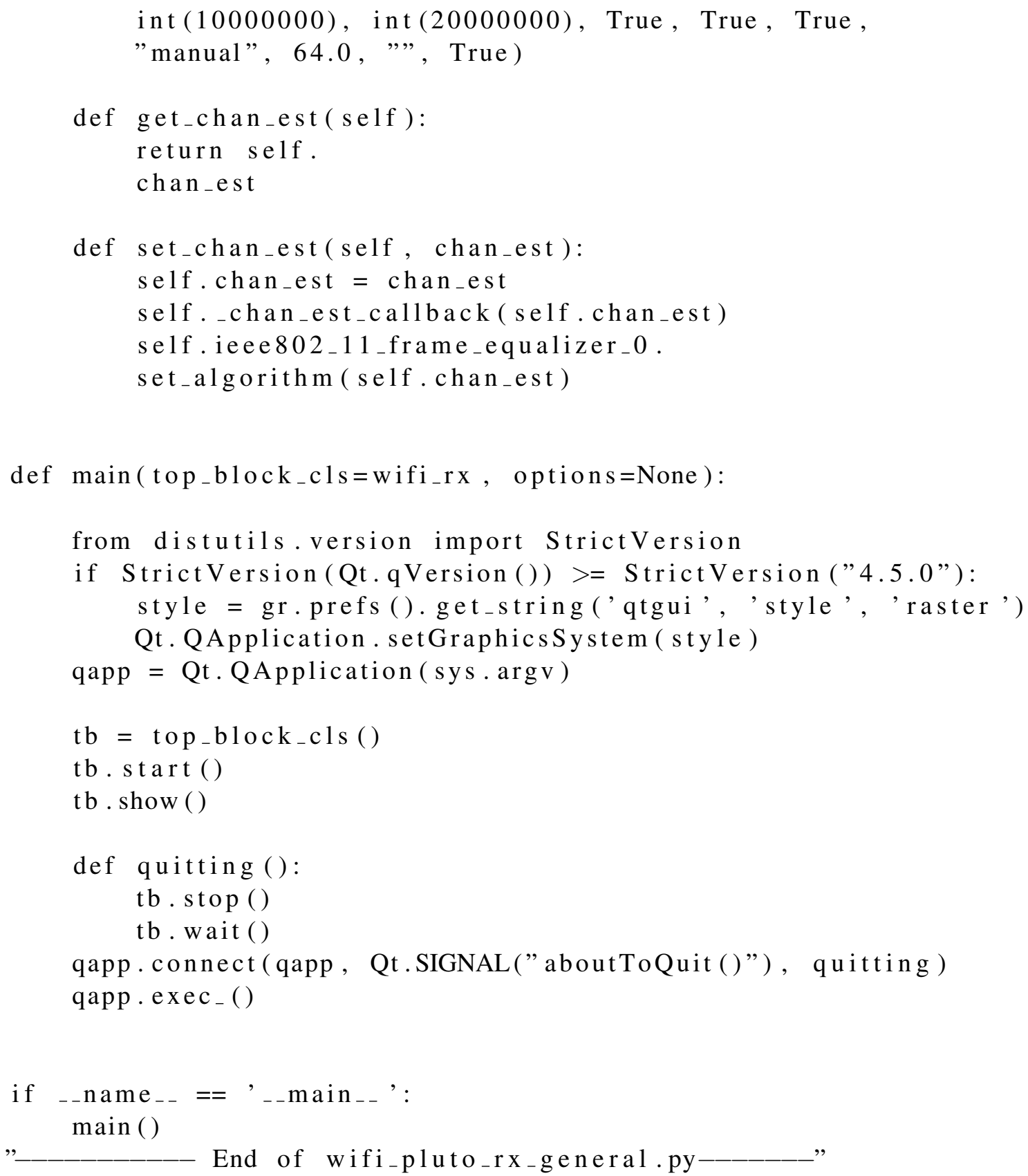

Note: All the scripts in Appendix B1 to Appendix B3 are customized exclusively for this Thesis and are mostly interdependent on each other. So, it is advisable to have all the scripts when executing the other. 


\section{APPENDIX C1}

\section{WORKING IMAGES}

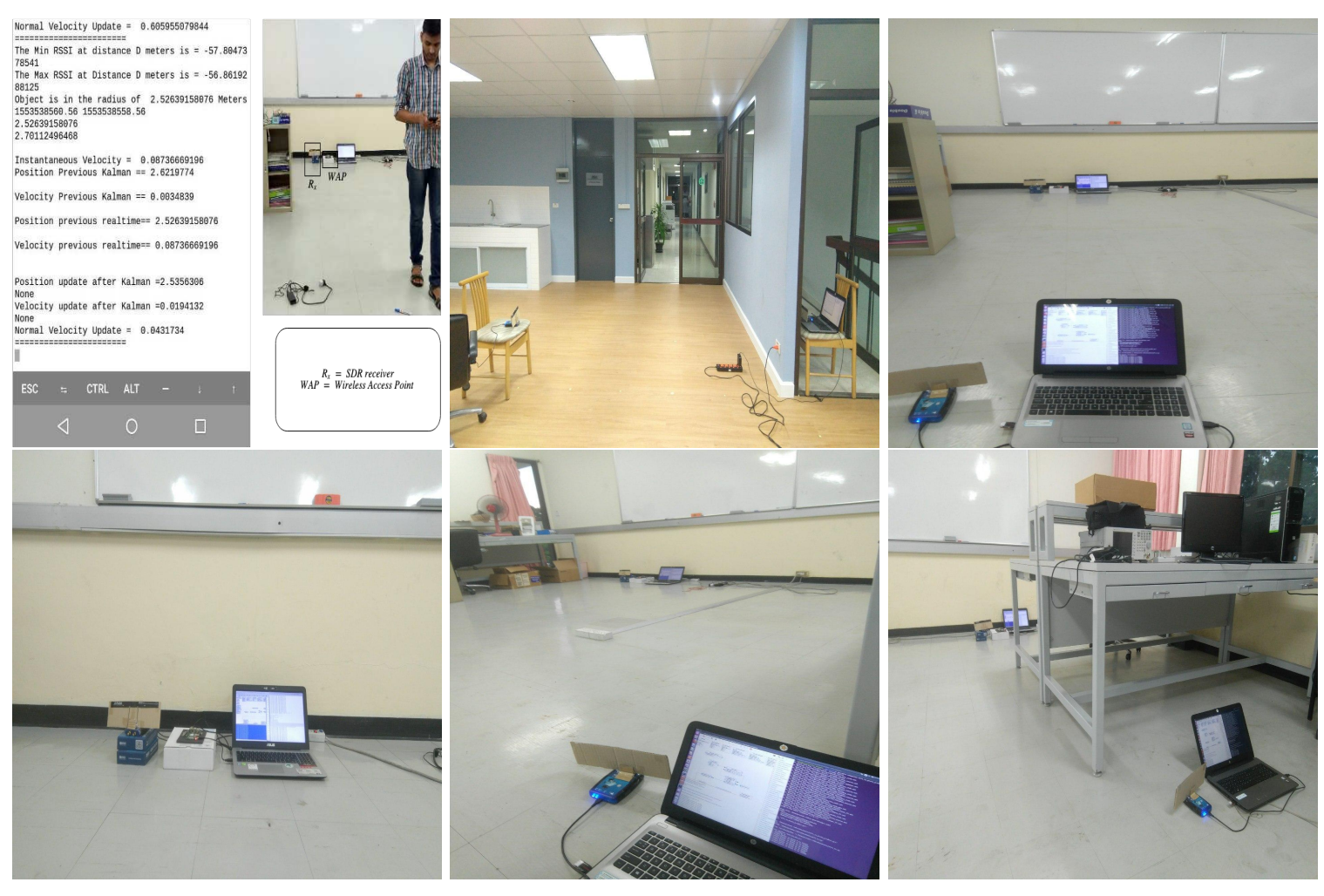

Figure A.1: Working Images

In Figure A.1: Figure A.1 shows working images at various stages of experimentation which are represented as images of $2 \mathrm{X} 3$ matrix.

- Figure A.1 $(1,1)$ : WAP based real-time RSSI and localization dashboard.

- Figure A.1 $(1,2)$ : IEEE $802.11 \mathrm{a} / \mathrm{g} / \mathrm{p}$ based localization when obstacle is between Rx \& Tx.

- Figure A.1(1 ,3): IEEE $802.11 \mathrm{a} / \mathrm{g} / \mathrm{p}$ based localization when Tx and Rx are in LOS.

- Figure A.1 $(2,1)$ : Experimentation set up with Raspberry Pi, SDR Rx and Laptop.

- Figure A.1(2, 2): IEEE $802.11 \mathrm{a} / \mathrm{g} / \mathrm{p}$ based localization when Tx and Rx are in diagonal.

- Figure A.1(2, 3): IEEE $802.11 \mathrm{a} / \mathrm{g} / \mathrm{p}$ based localization when Tx and Rx are in high clutter region. 

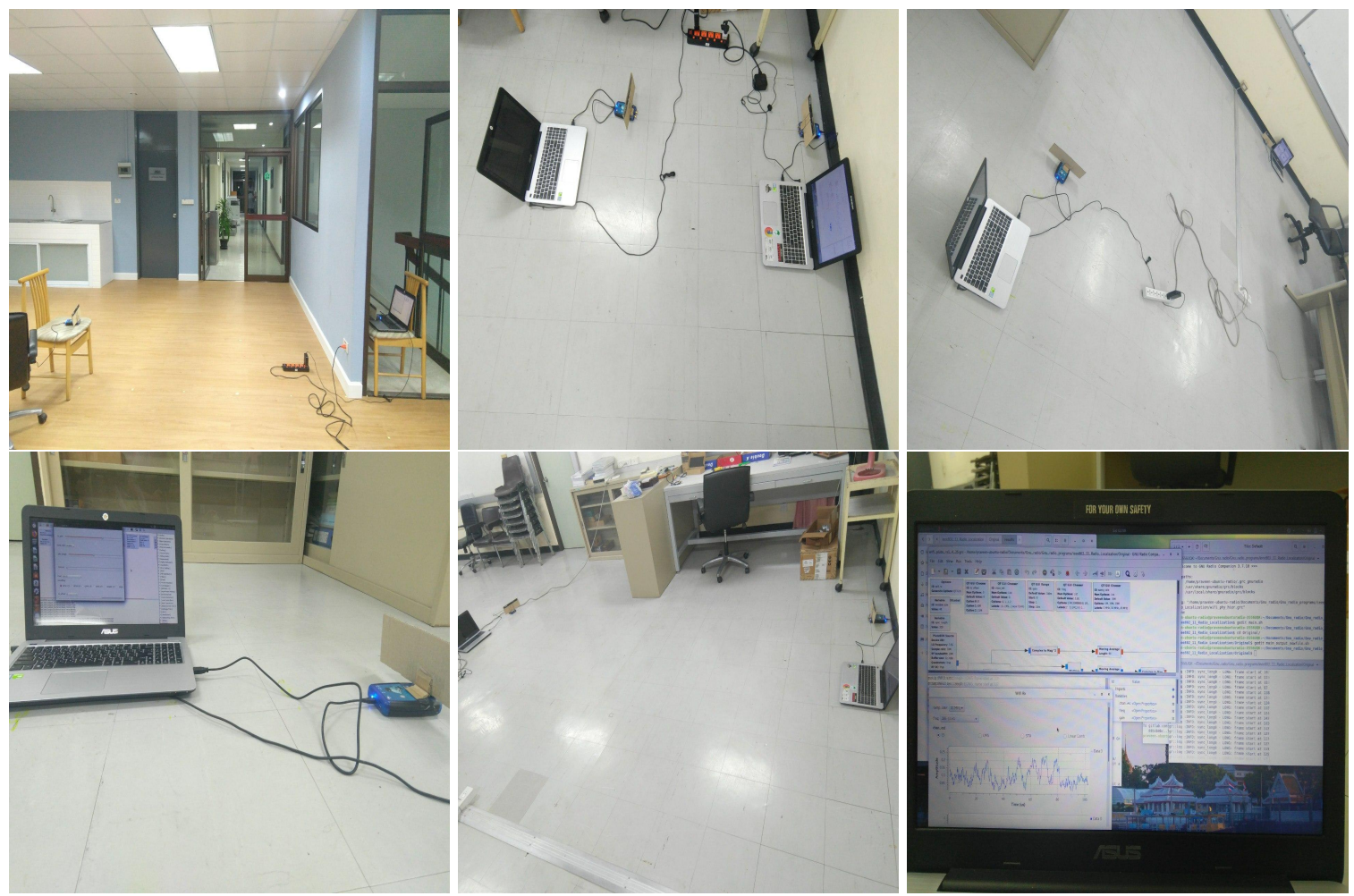

Figure A.2: Working Images

In Figure A.2: Figure A.2 shows working images at various stages of experimentation which are represented as images of $2 \mathrm{X} 3$ matrix.

- Figure A.2(1, 1): IEEE $802.11 \mathrm{a} / \mathrm{g} / \mathrm{p}$ based localization when obstacle is between Rx \& Tx.

- Figure A.2(1, 2): IEEE $802.11 \mathrm{a} / \mathrm{g} / \mathrm{p}$ localization when Tx and Rx are at near LOS.

- Figure A.2(1, 3): IEEE $802.11 \mathrm{a} / \mathrm{g} / \mathrm{p}$ localization when Tx and Rx are at high clutter diagonal.

- Figure A.2(2, 1): IEEE $802.11 \mathrm{a} / \mathrm{g} / \mathrm{p}$ localization shows Tx at high clutter region.

- Figure A.2(2, 2): IEEE $802.11 \mathrm{a} / \mathrm{g} / \mathrm{p}$ localization when Tx and Rx in LOS.

- Figure A.2(2,3): Screen Showing the software setup and RSSI reception from Tx. 


\section{APPENDIX D1}

\section{NOTABLE PEOPLE}

\section{Dr. Teerapat Sanguankotchakorn}

(D.Eng, Tokyo Institute of Technology, Japan,)

Associate Professor \& Head, Telecommunication Engineering

Asian Institute of Technology, Thailand

https://set.ait.ac.th/faculty/teerapat-sanguankotchakorn/

\section{Dr. Poompat Saengudomlert}

(Ph.D, Massachusetts Institute of Technology(MIT), USA)

Adjunt Professor, Telecommunication Engineering

Asian Institute of Technology, Thailand

https://set.ait.ac.th/faculty/poompat-saengudomlert/

\section{Dr. Attaphongse Taparugssanagorn}

(D.Tech, University of Oulu, Finland)

Associate Professor, Telecommunication Engineering

Asian Institute of Technology, Thailand

https://set.ait.ac.th/faculty/attaphongse-taparugssanagorn/

\section{Ms. Premma Rao}

Administrative Secretary, Telecommunication Engineering Asian Institute of Technology, Thailand

https://set.ait.ac.th/staff/

\section{Mr. Rajesh Kumar Dehury}

Technical Lab Supervisor, Telecommunication Engineering Asian Institute of Technology, Thailand

http://intranet.ait.ac.th/advsearch/Employee_search

\section{Dr. L. Pratap Reddy}

(Ph.D, Jawaharlal Nehru Technological University Hyderabad, India)

Professor, Electronics and Communication Engineering

JNT University Hyderabad (JNTUH) - College of Engineering Hyderabad, India http://jntuhceh.ac.in/faculty_details/4/dept/337 